\title{
Programmable Stimuli-Responsive Actuators for Complex Motions in Soft Robotics: Concept, Design and Challenges
}

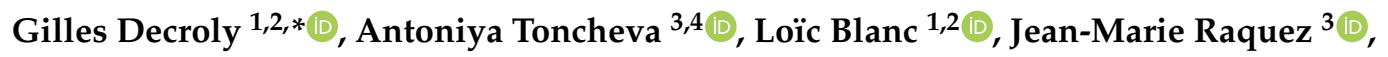 \\ Thomas Lessinnes ${ }^{1}$ (D), Alain Delchambre ${ }^{2}$ (D) and Pierre Lambert ${ }^{1, *(D)}$ \\ 1 TIPs Dpt CP 165/67, Université Libre de Bruxelles, 50 Avenue FD Roosevelt, B-1050 Brussels, Belgium; \\ Loic.Blanc@ulb.be (L.B.); Thomas.Lessinnes@ulb.be (T.L.) \\ 2 BEAMS Dpt CP 165/56, Université Libre de Bruxelles, 50 Avenue FD Roosevelt, B-1050 Brussels, Belgium; \\ alain.delchambre@ulb.ac.be \\ 3 Laboratory of Polymeric and Composite Materials, University of Mons, 23 Place du Parc, \\ B-7000 Mons, Belgium; antoniya.toncheva@umons.ac.be (A.T.); jean-marie.raquez@umons.ac.be (J.-M.R.) \\ 4 Laboratory of Bioactive Polymers, Institute of Polymers, Bulgarian Academy of Sciences, \\ Acad. G. Bonchev St., 103A, 1113 Sofia, Bulgaria \\ * Correspondence: gilles.decroly@ulb.ac.be (G.D.); pierre.lambert@ulb.ac.be (P.L.)
}

Received: 30 September 2020; Accepted: 3 December 2020; Published: 5 December 2020

\begin{abstract}
During the last years, great progress was made in material science in terms of concept, design and fabrication of new composite materials with conferred properties and desired functionalities. The scientific community paid particular interest to active soft materials, such as soft actuators, for their potential as transducers responding to various stimuli aiming to produce mechanical work. Inspired by this, materials engineers today are developing multidisciplinary approaches to produce new active matters, focusing on the kinematics allowed by the material itself more than on the possibilities offered by its design. Traditionally, more complex motions beyond pure elongation and bending are addressed by the robotics community. The present review targets encompassing and rationalizing a framework which will help a wider scientific audience to understand, sort and design future soft actuators and methods enabling complex motions. Special attention is devoted to recent progress in developing innovative stimulus-responsive materials and approaches for complex motion programming for soft robotics. In this context, a challenging overview of the new materials as well as their classification and comparison (performances and characteristics) are proposed. In addition, the great potential of soft transducers are outlined in terms of kinematic capabilities, illustrated by the related application. Guidelines are provided to design actuators and to integrate asymmetry enabling motions along any of the six basic degrees of freedom (translations and rotations), and strategies towards the programming of more complex motions are discussed. As a final note, a series of manufacturing methods are described and compared, from molding to $3 \mathrm{D}$ and $4 \mathrm{D}$ printing. The review ends with a Perspectives section, from material science and microrobotic points of view, on the soft materials' future and close future challenges to be overcome.
\end{abstract}

Keywords: soft robotics; smart materials; transducer; kinematics; mechanical programming; complex motion; $3 \mathrm{D}$ and $4 \mathrm{D}$ printing

\section{Introduction}

Nowadays, soft matter has gained increased interest as source material for the fabrication of robotic devices with a large range of applications. Conventionally, classic robotics systems are 
made of rigid connections and actuators, allowing high forces to be applied and accuracy in motion. However, the main limitations are the restricted degrees of freedom and the potential danger during the process of human-machine interaction. In this aspect, soft actuators are proposed as a suitable alternative to solve these problems, ensuring a safe and flexible actuation solution [1,2]. Their main advantage is that they are made of flexible materials and are based on deformation for actuation. Currently, a vast set of soft materials and kinematics are described, and new solutions are still evolving to overcome the challenges of the field [3]. On the other side, it is actually the generation of such numerous solutions that is presenting a difficulty, from a materials point of view, to properly select the most suitable solution for a given device functionality and application ranging from flexible medical devices to industrial grippers [4,5]. An additional complexity is the technological transfer from fundamental research to industrial application. The main question to be answered, "What is the next step?", is often faced by material scientists once a new transducer is fully developed and optimized, where the choice of the device's proper use remains unclear. On the other side, mechanical engineers require new materials indispensable for alternative and innovative solutions.

This review targets encompassing and rationalizing a framework which will help the robotics audience to understand, sort and design future soft actuators enabling complex motions, defined here as motions or deformations beyond pure elongation, shear, twist or bending. Special attention is paid to the last progress made on the development of innovative stimulus-responsive materials and complex motions programming for soft robotics. Mechanical programming refers here to the programming of an actuator's kinematics by embedding asymmetry into its material, components, design and/or fabrication process. With this, the authors aim to provide to the reader a clear overview on the possibilities offered by the soft transducers and to inspire and challenge the scientific community to go one step forward in the design of soft actuators. As a final remark, a great palette of soft robotics applications are illustrated.

An overview of the methodology to develop soft actuators capable of complex motions using soft smart materials is described in Figure 1. To generate a motion, energy is supplied to a transducer with a stimulus, typically heat, light or pressure. Under this stimulus, the transducer or stimuli-responsive material will produce mechanical work, i.e., force or displacement (Section 2.1). To program kinematics beyond isotropic expansion, asymmetry or anisotropy must be introduced. This structuring/patterning is a key aspect of the design methodology for soft robots, and this review is structured from this perspective. It can be introduced at several levels. The stimulus can even be isotropic or directed/inhomogeneous (gathered here under the term anisotropic) (Section 2.2). It can also come from the transducer or material itself (Section 2.3): isotropic transducers will deform uniformly under a stimulus, where anisotropic transducers present an intrinsic asymmetry. Finally, passive or active mechanical constraints can be added, called extrinsic asymmetry (Section 2.4). This additional level of anisotropy is added when building the actuator to guide the transducer deformation into the desired kinematics.

Comparing actuators, the relevant criteria and quantities, aside from the kinematics capabilities, are listed in Table A1. This synthesis is inspired from Hines et al. [3] and completed by other criteria and quantities reported in the reviewed works. Based on these, the transducers and asymmetry principles are compared quantitatively in Section 2.5 and their advantages and disadvantages are discussed.

From the obtained kinematics and towards the generation of complex motions, i.e., when building the actuator into the rest of the robot body, several mechanical programming strategies are described in the literature (Section 3.1). A wide set of complex motions can be obtained, strongly related to the targeted application (Section 3.2). Transversely, the proposed fabrication methods proposed are described and compared (Section 4). These fabrication methods cover the synthesis of the transducers, the manufacturing of actuators capable to perform basic kinematics or the fabrication of integrated robots capable to perform complex motions and aim to be more and more integrated, with the rise of 4D printing. Perspectives on the future challenges of the field conclude this review (Section 5). 


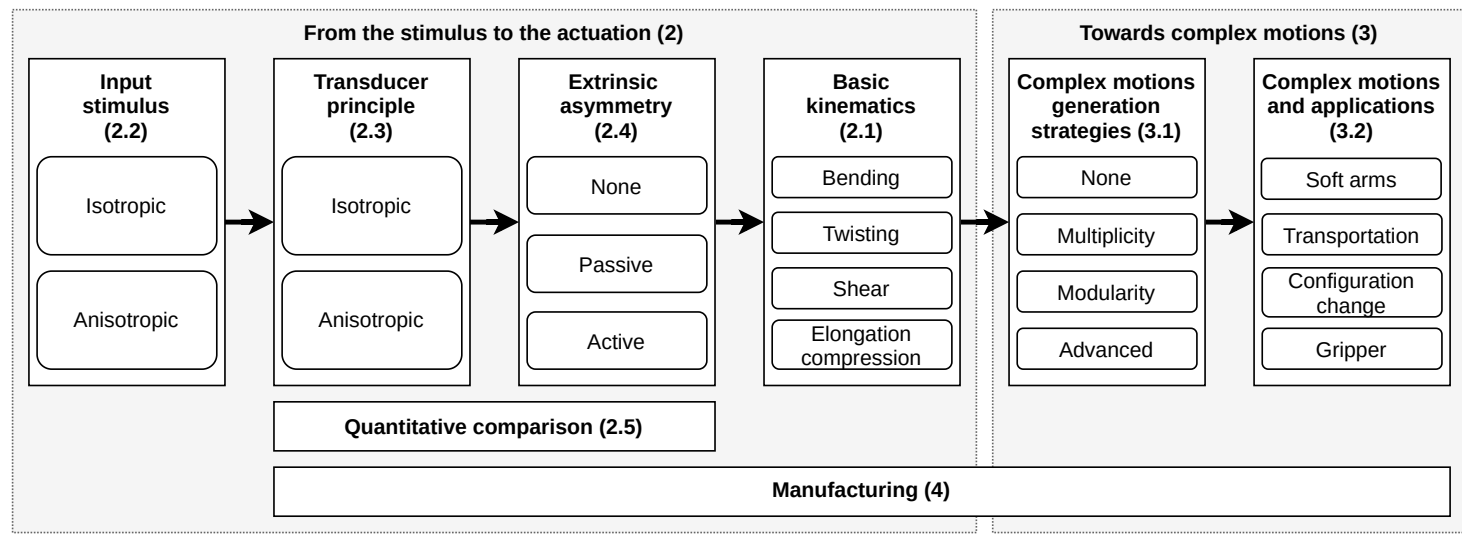

Figure 1. Schematic overview of this review corresponding to the proposed framework towards the mechanical programming of stimuli-responsive soft robots capable of complex motions: the numbers in brackets refer to the dedicated section.

\section{From Stimulus to Actuation}

Under the framework described in Figure 1, an overview of the literature solutions to generate a desired basic kinematic is presented in Figure 2. This representation aims to give an overview of the different roads to introduce anisotropy in the actuator to generate a given kinematics. Moreover, it allows the reader to identify the most widely explored strategies and to open new perspectives. In this representation, a thermally actuated bending actuator made of a bilayer, with one expansive and one passive layer, and heated using the Joule effect would be represented by the following set of arrows: current to heat, heat to thermal expansion, thermal expansion to multi-material and multi-material to bending. If multiple stimuli, transducers or extrinsic asymmetry principles are used, the actuator will be represented by a set of parallel arrows. Every complex motion can be seen as a combination of four basic kinematics given in Figure 3. A similar roadmap towards the generation of complex motions from the basic kinematics will be presented in Section 3.

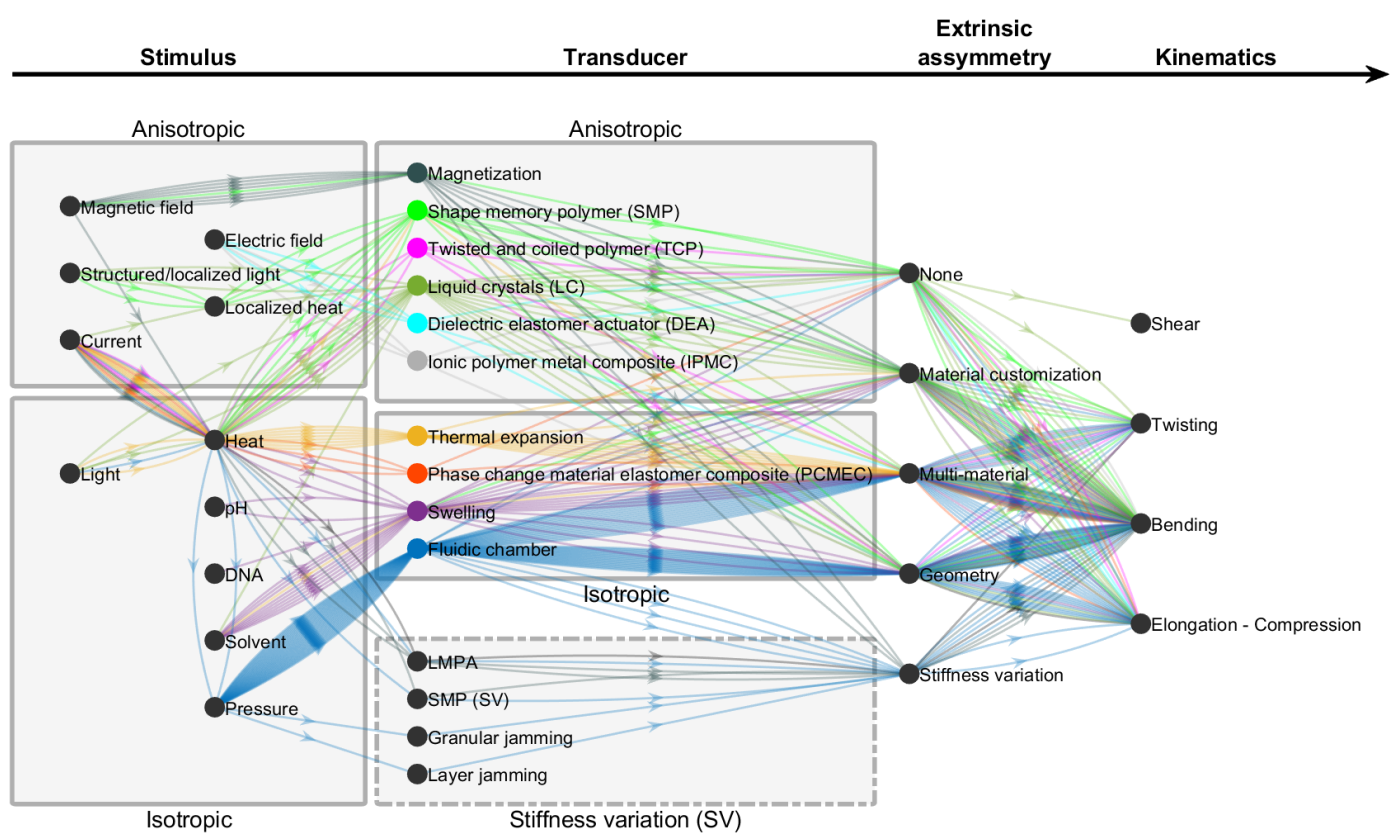

Figure 2. Roadmap of actuator design from stimulus to actuation: each actuator reviewed is represented by a set of arrows. The arrow colors indicate the main transducer principle used to generate a deformation. Actuators using several stimuli, transducers, or extrinsic asymmetry or are able to generate several kinematics are represented by several arrows. 

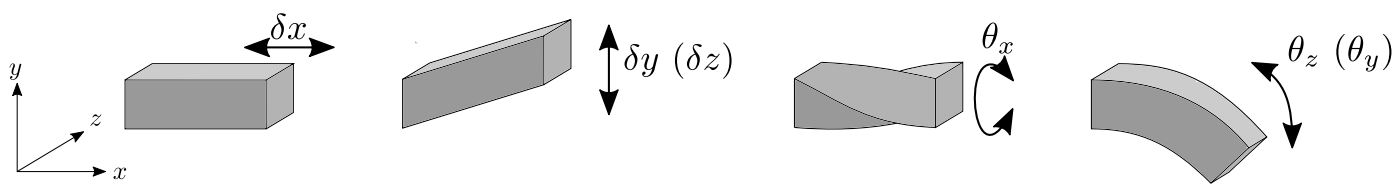

Figure 3. Four basic actuators kinematics can be define, corresponding to the six degrees of freedom of a surface when considering the other side fixed: three translations (elongation/compression $\delta_{x}$ and shear $\delta_{y}$ and $\delta_{z}$ ) and three rotations (twisting $\theta_{x}$ and bending $\theta_{y}$ and $\theta_{z}$ ).

\subsection{Basic Kinematics}

The actuation can be sorted into four basic kinematics: bending, twisting, elongation/compression and shear (Figure 3). When considering a beam with a fixed extremity, it corresponds to the six degrees of freedom of the other extremity. Note that the decomposition of complex motions into these basic kinematics can seem artificial. Numerous works present actuators directly able to combine these kinematics. Helixing, a combination of bending and twisting, is the most common example. Furthermore, "pure" kinematics are rarely obtained-small elongation of twisting actuators is often observed. However, this decomposition into basic kinematics allows for the presentation of a coherent framework and the comparison of the proposed solutions.

In mechanics, the generated mechanical work of an actuator can be characterized using the generated stress $\sigma$ and strain $\epsilon$, independently of the geometry of the structures. This paragraph describes simple models to obtain these normalized quantities from the developed forces and deformations of linear and bending actuators. Note that similar models for shear and twisting are not detailed here, since shear is rarely reported and twisting is often described as being derived from bending by varying the actuator design. In the case of free deformation, the actuation energy will generate a maximal elongation. If constraints are added to the structure, the mechanical work will be transformed in both force and elongation. Finally, if the deformation is fully constrained, the generated force will be maximal. The longitudinal (engineering) stress is defined as the ratio of the longitudinally generated force $F$ over the surface $A: \sigma=F / A$ (Figure 4a). In this case, the stress is uniform over the section. The longitudinal strain is given by the ratio of the length change $\Delta L$ over the initial length $L_{0}: \epsilon=\Delta L / L_{0}=\left(L-L_{0}\right) / L_{0}$ (Figure $4 \mathrm{~b}$ ). When the actuator deforms in bending, the (engineering) stress is nonconstant over the section and increases linearly from the neutral axis to the external fibers of the structure, as illustrated in Figure 4c. By definition, the neutral axis is the position at which the bending stress is null (and changes its sign). During bending, there is an equilibrium of longitudinal forces, but the nonconstant and non-null stress result in a bending moment. In this case, the actuation stress can be obtained from blocking force results. The actuator can be considered a beam exerting a force $F$ perpendicular to its extremity. The actuation stress can be obtained in the most constrained fibre (at a distance $d$ from the neutral axis) of the actuator: $\sigma=F L_{0} d / I_{z}$, where the second moment of area $I_{z}$ characterizes the geometrical properties of the actuator. Several quantities can be used to characterize the bending deformation (Figure $4 \mathrm{~d}$ ). Contrary to the bending angle $\alpha$, the curvature $k=\alpha / L$ (or the radius of curvature $R_{c}=l / \alpha=L / k$ ) is independent of the length $(L)$ of the actuator. Computing an actuation strain value from the curvature of the actuator allows us to generalize the results and to compare the developed actuator to the literature. The strain is computed under the assumption of a constant curvature, which is often verified, and can be obtained from the most elongated fibre (at a distance $d$ from the neutral axis) of the actuator: $\epsilon=\left(L-L_{0}\right) / L_{0}=\left(L_{0}+d \alpha-L_{0}\right) / L_{0}=d \alpha / L_{0}=k d$. The longitudinal stiffness, also known as Young's modulus $E$, is obtained generally defined by the initial slope of the stress-strain curve when the structure is subjected to an external loading. The reviewed solutions are compared according to these normalized quantities in Section 2.5. 


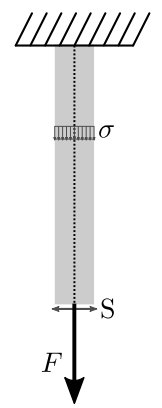

(a) Linear force.

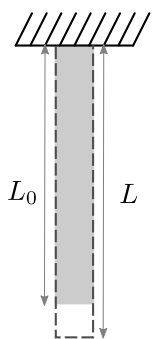

(b) Linear elongation.

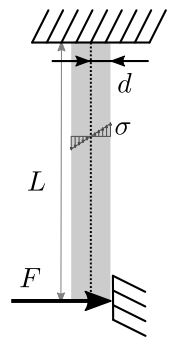

(c) Blocking force.

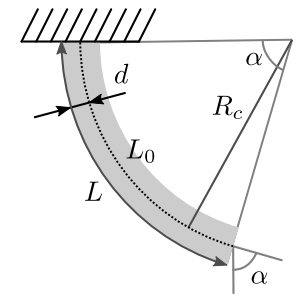

(d) Bending.

Figure 4. Stress and strain for linear elongation and bending: the dashed lines are the neutral fibres.

\subsection{Input Stimuli}

Before describing further the stimuli-responsive transducers used in soft robotics, this section presents a brief overview of the stimulus used. The stimulus provides the actuator energy which the transducer will transform in mechanical work (Figure 2). This energy can be of various forms and can be sorted according to its anisotropy. Isotropic stimuli, such as mechanical (pressure), electromagnetic radiations (light), thermal (heat) or chemical ( $\mathrm{pH}$, solvent, etc.) stimulus, do not have an intrinsic directionality. Contrarily, anisotropic stimuli such as electric or magnetic fields will generate anisotropic deformation of the transducer. Using structured light or localized heat or light has also been reported to embed the directionality of the actuator in the stimulus itself. Currently, another directed stimulus is commonly used, but not for its anisotropy (Figure 2): it has only been reported as a precursor to heat.

The choice of the stimulus is strongly linked to the selected transducer, and their use will be discussed along with presentation of the different transducers (Section 2.3). Moreover, the advantages and limitations of a solution are more related to the stimulus-transducer couple than to the stimulus itself. Different strategies can be used to bring energy under a temporary form to the transducer. Several works reported the use of current or light to generate a temperature increase thanks to the Joule or photothermal effect. This will be further discussed later in the next section.

\subsection{Transducer Principle}

When designing an actuator, the choice of the transducing principle will generally be determinant and will guide the rest of the development. It will generally impose a stimulus and the possible need of extrinsic asymmetry. Furthermore, the fabrication processes, costs and mechanical capabilities of the actuators depend on their transducing principles. The different transducers are compared in Section 2.5 according to the criterion defined in Table A1 and in Section 4 according to the fabrication methods.

Here, we define the transducer as the material or system that will transform a stimulus energy into mechanical deformation. The transducer is then a general nomenclature, counting stimuli-responsive materials (active/smart matter) or structures (fluidic chambers and dielectric elastomers). These transducers can even be isotropic, meaning that they will deform uniformly under a stimulus, or anisotropic, if they present an intrinsic asymmetry. The isotropic transducer will require coupling with extrinsic asymmetry to guide the deformation into a given kinematic. Based on this criterion, a general classification of the transducers is proposed in Table 1. This review focuses on intrinsically soft stimuli-responsive transducers. Other mechanical transducers are briefly mentioned in this section but are not included in the comparison. Aside from the mechanical transducers, two complementary stimuli-responsive materials or systems are described: stiffness variation transducer, that does not provide mechanical work but a change in the structure stiffness, and what we call here nonmechanical transducers, transforming the energy from a given stimulus to another. 
Table 1. Stimuli-responsive transducers for soft robotics, classified as a function of their intrinsic asymmetry: isotropic transducers will deform isotropically when subjected to a stimulus, contrary to anisotropic transducers. Typical materials used are also indicated.

\begin{tabular}{|c|c|c|}
\hline & Classification & Typical Main Materials \\
\hline \multirow{4}{*}{$\begin{array}{l}\text { Isotropic } \\
\quad- \\
\text { undirected }\end{array}$} & Fluidic chamber & $\begin{array}{l}\text { Elastomers (Ecoflex [6-16], Dragon Skin [17], } \\
\text { Sylgard 184 [18,19], other [20-25]), fabric [15,26,27], } \\
\text { Filaflex [28], hydrogels [29], Diels-Alder } \\
\text { polymer [30], polypropylene [31], FC72 fluid [24], } \\
\text { ethanol [16], paper [32], urethane [27], } \\
\text { gelatin-based biogel [33] }\end{array}$ \\
\hline & $\begin{array}{l}\text { Phase Change Material Elastomer } \\
\text { Composite (PCMEC) }\end{array}$ & $\begin{array}{l}\text { Elastomers [34-36], phase change material } \\
\text { (wax [35], ethanol [34]) }\end{array}$ \\
\hline & Thermal expansion & $\begin{array}{l}\text { Elastomers [37-39], resin [40,41], } \\
\text { SMP (MPCL) [42], other polymer [43], } \\
\text { paper [43,44], micro- and nanofillers [45] }\end{array}$ \\
\hline & Swelling & $\begin{array}{l}\text { Hydrogels [46-50], paper [43], LCN [51], } \\
\text { SMP [48,52-55], cross-linked PCL [56], } \\
\text { other polymers [43,57-64] }\end{array}$ \\
\hline \multirow{6}{*}{$\begin{array}{c}\text { Anisotropic } \\
- \\
\text { directed }\end{array}$} & Shape Memory Polymer (SMP) & $\begin{array}{l}\text { Resins [65], functionalized PCL [66], } \\
\text { functionalized PLA [67], polyurethan, wax [48], } \\
\text { DiAPLEX [68], other polymers [69,70] }\end{array}$ \\
\hline & Magnetization & $\begin{array}{l}\text { Elastomers [71-73], resin [74], SMP (DiAPLEX [68], } \\
\text { other [74]), low melting point alloy (LMPA) } \\
\text { (Cerrolow 117) [75], magnet }[75,76] \text {, magnetic } \\
\text { particles [68,71-74] }\end{array}$ \\
\hline & Liquid Crystal (LC) & LCE [77-84], LCN [85-87] \\
\hline & Dielectric Elastomer Actuator (DEA) & Elastomers [88], LCE [89], polyethylene [90] \\
\hline & Twisted and Coiled Polymer (TCP) & Nylon 6,6 [91-93], polyethylene [91] \\
\hline & $\begin{array}{l}\text { Ionic Polymer Metal Composite } \\
\text { (IPMC) }\end{array}$ & $\begin{array}{l}\text { Electrolyte membrane (Nafion [94-96], } \\
\text { Flemion [97], Aciplex [97]), electrode (gold [94], } \\
\text { gold-chromium [95], palladium [96], } \\
\text { platinum [97]) }\end{array}$ \\
\hline
\end{tabular}

Several recent works reviewed soft transducers principles. Hines et al. [3] and Yang et al. [98] proposed complete reviews of soft transducers' working principles and capabilities [3,98]. Other works focused on the material aspects [2,99,100], on their configurations and applications [101], on untethered soft microrobots [102,103] or on printable transducers [104]. Other capabilities of soft actuators and transducers, as sensing, self-healing, and growing have also been reviewed $[105,106]$. For a complete overview of smart stimuli-responsive materials and their use in soft robotics, the authors redirect the reader to Koshima [107].

\subsubsection{Soft Anisotropic Transducers}

Shape memory polymers (SMPs) are part of an emerging class of new materials called "shape-memory composites" that include polymers, alloys, hybrids, ceramics and gels. The great advantage of SMPs is their ability to generate complex motions accompanied with large stiffness changes upon heating and a multitude of desirable properties, such as: low density, recycling, high recoverable strain within a wide range of stimuli, material transparency, and chemical stability upon actuation. Of interest are also the numerous chemical modifications for tunable final properties and processing procedures (blends, extrusion, thermal compression, etc.), biocompatibility and biodegradability with an opportunity to adjust the degradation rate [108]. SMPs are first produced in an initial (permanent) shape. Once activated by their stimulus (mainly heat), they can be programmed in a 
new temporary shape. Upon exposure to an appropriate stimulus, the polymer is activated and tends to recover its initial shape. Despite the multitude of shapes to be adopted, one of the major SMP drawbacks is related to their low tensile strength, restricted heat actuation and lack of proper function. They are known to have a low thermal conductivity and to be inert to electrical, light and electromagnetic stimuli, which restricts their domain of application. However, these difficulties can be overcome with the incorporation of (in)organic (nano)fillers (nanotubes, nanofibers, nanopartciles (NPs), nanospheres, nanorods and others) within the polymer matrix for the generation of shape-memory (nano)composites (SMCs). These fillers offer the advantage to be characterized with high specific surface area, stiffness and various functionalities (electrical conductivity, light and magnetic sensibility, solvent and water responsiveness, etc.). As a result of the conferred functionality, multi-stimuli-responsive materials can be obtained, with potential application in intelligent medical devices [109], flexible electronic devices [110], sensor and actuators [111], and self-deployable and self-folding structures in spacecrafts [112,113]. Therefore, in order to use these polymers as actuators in soft robotics, they have to be initially programmed. Depending on the degree of shapes to be acquired, the SMP can "memorize" one or multiple temporary shapes for "one-way" or "two-way" actuation, respectively. "One-way" SMPs are defined as materials capable of memorizing one temporary shape at a time, with highly controllable irreversible shape recovery in a one-way manner (multi-shape materials). "Two-way" SMPs are represented by programmable and reversible shape switch between two distinct shapes. The "two-way" shape-memory effect of SMPs is defined as a phenomenon occurring during the heating and cooling step in the presence or absence of external stress due to the reversible conformations of the polymer chains. Such polymer materials are currently considered as having actuator behaviour and are represented mainly by morphological characteristics such as crosslinked semi-crystalline polymers [114-116] and glass-forming polydomain nematic networks [117]. The authors redirect the reader to Pilate et al. [118] for a complete review of the chemical aspects of SMP actuation [118]. Recently, several strategies have been developed to allow cycle actuation. Scalet [119] and Xia et al. [120] reviewed recent advances and capabilities of SMP for soft robotics applications [119,120]. Such transducers were demonstrated with several materials, such as resins [65], functionalized Polycaprolactone (PCL) [66], functionalized Polylactide (PLA) [67], polyurethan, wax [48], DiAPLEX [68] or other polymers [69,70]. The actuation temperature also depends on the polymer properties and range typically between $37^{\circ} \mathrm{C}$ [66] and $200^{\circ} \mathrm{C}$ [67]. Using photothermal effect is another promising solution to remotely actuate SMPs and other heat-responsive transducers using light. This is further discussed in Section 2.3.5. As discussed in Section 2.5, SMP are generally made of relatively stiff materials. Their advantages rely in their multiple actuation modes described above, but until today, most of the demonstrated solutions show a relatively slow actuation of the order of magnitude of the second, limited by the thermodynamics of the actuation. Moreover, compared to other solutions and when reported, the actuation stress is relatively low.

Magnetization transducers rely on the torque generated on dipoles or magnetization profile when placed in a magnetic field. Yang and Zhang [121] reviewed microrobots using magnetic transducers [121], and Sitti and Wiersma [122] compared magnetic and optical actuators [122]. The first class of magnetic transducers embed a magnetization profile (Figure 5a) [71-74,123] made of magnetic microparticles embedded in a soft matrix. The magnetization direction was generally controlled during the fabrication process. Elastomer [71,73] and UV curable resin [123] have been reported as main matrix materials. Ze et al. [74] demonstrated the possibility to reprogram the magnetization profile using an SMP matrix [74]. Alternatively, the soft actuators can be actuated locally by embedded magnets [75,76]. Magnetization transducers show fast actuation time, up to around $10 \mathrm{~ms}$ [72]. This solution is also very promising to develop miniaturized actuators, with thickness as low as $30 \mu \mathrm{m}$ [73]. This untethered actuation relies on an external magnetic field, generated using permanent magnets or electromagnetic coil [122] and with an amplitude reported between $11 \mathrm{mT}$ [73] and $200 \mathrm{mT}$ [71], and is suitable for various environments. 
(a)

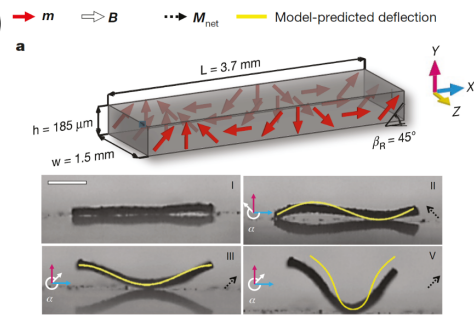

(d)

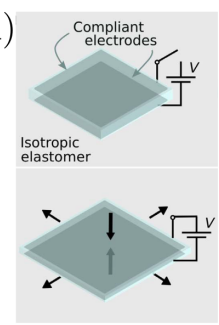

(b)

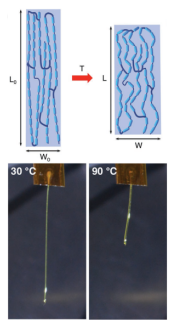

(e)

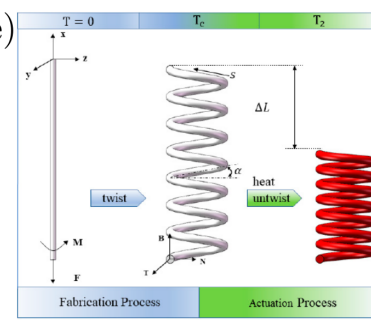

(c)

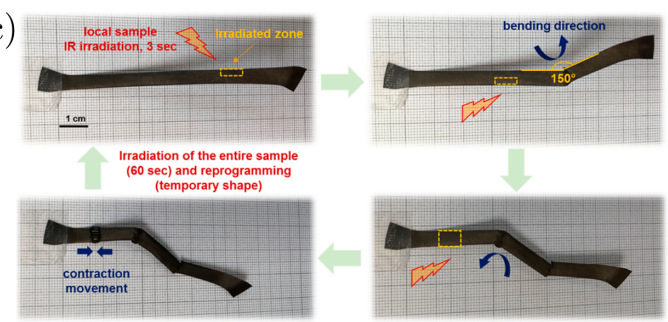

(f)

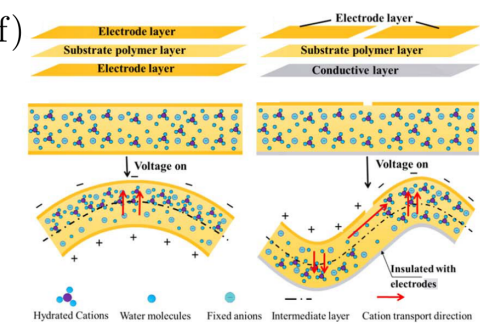

Figure 5. Examples of anisotropic transducers. (a) Magnetization profile [72]. Copyright 2018, Springer Nature. (b) Liquid crystal elastomer (LCE) thermoactuation [81]. Copyright 2017, John Wiley and Sons. (c) Shape Memory Polymer (SMP) [66]. Copyright 2018, American Chemical Society. (d) Dielectric elastomer actuator (DEA) [89]. Copyright 2019, American Association for the Advancement of Science. (e) Twisted and coiled polymer (TCP) [93]. Copyright 2020, MDPI. (f) Ionic polymer-metal composite (IPMC) [94]. Copyright 2018, IOP Publishing Ltd.

Liquid crystals (LCs) have been widely studied and present various stimulation modes. In polymer science, the most attractive class is the LC elastomers (LCE) made of crystal molecules (mesogens) that are appended to a compliant polymer backbone. For example, a soft backbone allows the mesogens to be easily reoriented, thus preventing free flow of the polymer macromolecules. In addition, mesogen reorientation can induce stresses along the backbone that result in bulk strain of the LCE accompanied with thermal or electrical response. Often, heat diffusion leads to slow thermal activation of the LCE that requires an active cooling step [124]. The incorporation of magnetic fillers (ferroelectric LC) brings an intrinsic polarization of the mesogens. In addition, a polymer matix with a higher degree of cross-linking (described as LC networks, LCN) led to an increase in the material stiffness compared to analogous LCE. In the literature, several reviews focused attention on the actuation principles and LCE properties [125] and LCP [126,127]. Two important phases of LC are nematic or smectic, where the mesogens are respectively globally aligned or aligned and arranged in layers. By phase transition, an alternative isotropic phase can be obtained with no global order. In the case where the mesogens are aligned within the material volume, a directed contraction is observed during phase change in the direction of the originally aligned mesogens (Figure $5 b$ ). The related alignment can be obtained and controlled by adequate fabrication material processing. This way of introducing anisotropy in the transducer is further discussed in Section 2.4.3. Several works describing LCE [77-84] and LC polymer [85-87]-based actuators have been reviewed and are compared to other transducers in Section 2.5. An interesting feature of LC is that the phase change can be obtained using different stimuli. The three main actuation modes are thermal $[77-81,83]$, where the LC is directly heated; photothermal $[82,84,87]$, where heat is generated in situ using light; and photochemical $[85,86]$. Photothermal and photochemical actuation can occur in parallel $[82,85]$.

Dielectric elastomer actuators (DEAs) are a variety of electroactive polymers (EAPs) that can undergo shape deformation upon electrical stimulation accompanied with a sensitive change in the material size and shape (Figure 5d). Generally EAPs are categorized into two classes known as ionic (based on Coulomb forces present within the material) and electronic (ions mobility, e.g., DEA and LCE). Other materials with electroactive properties are the electroactive ceramics and electroactive shape memory alloys with limited application in soft robotics as well as McKibben actuators. In this context, EAPs gained attention for their flexibility and tunable mechanical properties. DEAs are generally composed of an elastomer layer sandwiched between two electrodes. When subjected to 
an electric field, depending on the material properties, a given degree of compression can be reached between the electrodes leading to an anisotropic deformation. Of great importance here is the material elastic characteristics, softness and stretchability. The incorporation of electrically conductive fillers can additionally enhance actuator performance. Isotropic elastomers [88,90] are generally used as dielectric materials. Davidson et al. [89] used anisotropic LCE to obtain high directed deformations [89]. As discussed in Section 2.5, they presented a high actuation time relative to their size. Suo [128] et al. reviewed the different types of DEA and their theory [128], and Gupta et al. [129] focused on their use in soft robotics [129]. One of the most attractive EAP characteristics is their promising application as biological muscles (dielectric elastomer artificial muscles [130]) to emulate the operation of inherent vibration damping, high fracture toughness and large actuation strain.

Twisted and coiled polymers (TCPs) are structures made of shaped polymeric chains (Figure 5e). When heated, the entropy of the originally aligned polymeric chains increases, leading to a small contraction of the fibre. By twisting and coiling the nylon fibre, this contraction can be strongly amplified to exhibit high directed strains [91]. This class of transducers develops very high energy densities but at low efficiencies [91]. Most of the reported TCPs use Nylon 6,6 as the main material. The fibres can directly be silver coated for heating [93]. Other polymers, such as polyethylene, can be used [91]. TCP must generally be preloaded before actuation, but Sun et al. [92] demonstrated free-stroke capabilities enabled with an adapted fabrication process. Similar to most transducers, TCP can be used in various environments. An actuation time of $200 \mathrm{~ms}$ has been demonstrated, limited by the thermodynamics of the actuation [91]: Nylon 6,6 needs to be heated up to $140^{\circ} \mathrm{C}$ to complete the actuation. The dimensions are generally on the millimeter scale, related to the polymer fibre diameter. Up to 1 million actuation cycles have been demonstrated [91].

Ionic polymer-metal composites (IPMCs) are composed of a thin electrolyte membrane sandwiched typically between two electrodes made of noble metals (Figure $5 \mathrm{f}$ ). Under the application of an electric field, a displacement of cations associated with water molecules is observed towards the cathode. This generates local stress and induces a deformation of the structure. Hao et al. [97] proposed a compact and comprehensive review of recent advances in the field [97]. The classical IPMC design consists of an electrolyte membrane sandwiched between two electrodes. Typical materials are indicated in Table 1. Alternatively, Chang et al. [94] uses electrodes only on one side, opening new possibilities in terms of design. IPMCs present efficient energy conversion, require relatively low voltage ( $1 \mathrm{~V}$ to $7 \mathrm{~V}$ corresponding to electric fields of $5000 \mathrm{~V} / \mathrm{M}[94,95]$ to $1200 \mathrm{~V} / \mathrm{M}$ [96]), and allow an easy miniaturization [97]. Thicknesses as low as $200 \mu \mathrm{m}$ have been reported [94]. IMPC transducers can be used in air or water [97]. The disadvantages of this transducer rely on the low output forces and the relaxation observed when water is used as solvent [97]. The actuation time, originally of several seconds, can be reduced to $190 \mathrm{~ms}$ by using a short pulse of high voltage [96].

\subsubsection{Soft Isotropic Transducers}

Fluidic chambers relate to fluidic or pneumatic actuators (Figure 6a). They have been widely studied and contribute to the growing interest in soft robotics. The deformation is obtained here thanks to the deformation of a chamber made of soft material under a fluidic or pneumatic pressure. From this deformation, various kinematics can be obtained, as described in Section 2.1. Several reviews focused on pneumatic and fluidic actuators for soft robotics [131-134]. Typical materials used are commercial hyperelastic silicones, used for their low cost, availability, and ease of shaping (Table 1). Alternatives have also been proposed. Yuk et al. [29] developed soft hydrogel-based hydraulic actuators [29], while other works used stiffer polypropylene bellows [31]. Baumgartner et al. [33] developed a biodegradable material and demonstrated its potential for pneumatic actuators [33]. Recently, several works proposed to use fabric coupled with a sealing layer to achieve high forces with simple and easy-to-manufacture designs [26,27]. The dimension of this solution remains mainly on the centimeter scale, even if there is no intrinsic limitation to the miniaturization [6]. However, Sinatra et al. [19] demonstrated a fluidic actuator of less than $0.5 \mathrm{~mm}$ in thickness [19]. 
Actuation time can be faster than $0.1 \mathrm{~s}$ [18], limited by the viscoelasticity of the material [135], the fluid dynamics of the pressure leads [18], and the pressure source. Joshi and Paik [136] presented a parameter optimisation for pneumatic supply systems [136]. The response time can also be improved using the release of the elastic energy stored by the elastomeric chamber to reach a recovery actuation time of around $50 \mathrm{~ms}$ [10]. Fluidic chamber solutions are compared quantitatively to other transducers along the mentioned criteria in Section 2.5. The pneumatic or hydraulic pressure commonly ranges between 0.2 bar and 1 bar, depending mainly on the actuator structural stiffness (material stiffness, geometry and dimensions), and is limited by the risk of bursting or leakage. Several works reported input pressures up to 3 bar [22,31]. The pressure lines in congestion remain an important limitation of fluidic chamber actuators in terms of miniaturization and tetherability. Several solutions have been proposed to overcome this limitation, as embedding pressure sources [137]. Alternatively, to avoid pressure lines, Matsuoka et al. [24] used the pressure increase caused by phase change of a fluid (FC-72) sealed in the fluidic chamber and heated by a cartridge heater [24]. Han et al. [16] proposed a similar solution based on near-infrared radiation heating [16]. Fluidic chamber transducers have been demonstrated in various environments and shown especially efficient for underwater actuation [29].
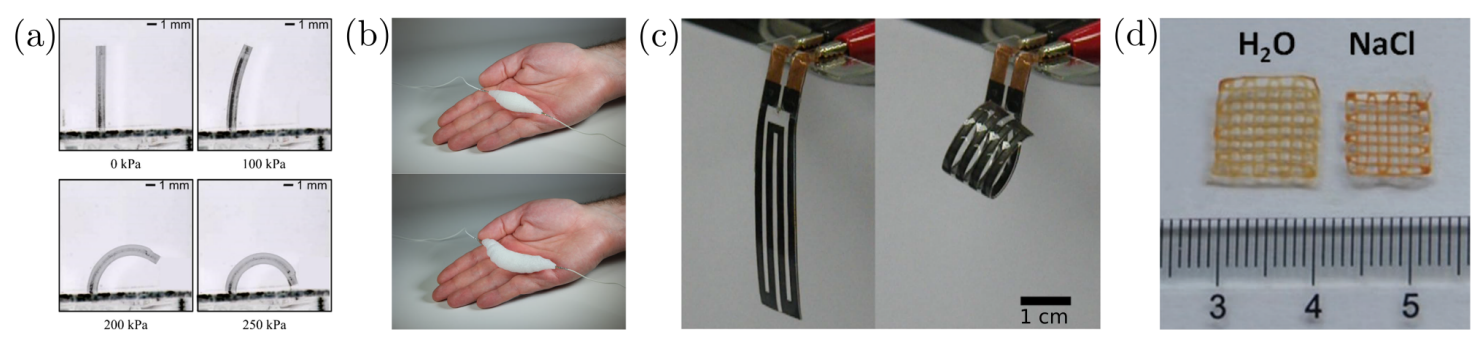

Figure 6. Examples of isotropic transducers. (a) Fluidic chambers [18]. Copyright 2018, Springer Nature. (b) Phase change material-elastomer composites (PCMEC) [34]. Copyright 2017, Springer Nature. (c) Thermal expansion [37]. Copyright 2018, IOP Publishing Ltd. (d) Swelling [50]. Copyright 2018, John Wiley and Sons.

Phase change material-elastomer composites (PCMECs) rely on the expansion of a phase change material embedded in inclusions of a soft elastomer matrix (Figure 6b). When increasing the temperature, the phase change will induce strong expansion of the matrix. Note that, in our classification, this class of transducers does not include material presenting a nonclassical change in state, such as LCs or SMPs. PCMECs using paraffin wax melting [35] and ethanol vaporization $[34,138,139]$ have been reported. The phase change is triggered by heat, with the temperature depending on the inclusion material (typically $78^{\circ} \mathrm{C}$ for ethanol). Demonstrated PCMEC actuator sizes are generally in the centimeter range [139]. For PCMECs relying on a fluid's vaporization, such as the material of Miriyev et al. [34], miniaturization is limited by diffusion of the phase change material out of the matrix [140] and bubbles size (100 $\mathrm{mm}$ to $1 \mathrm{~mm}$ [138]). Similarly, life time is also limited by diffusion, but Miriyev et al. [140] developed a rejuvenation strategy to overcome this limitation [140]. The advantages of PCMEC rely in the high energy density developed [34,35]. The actuator stiffness depends mainly on the elastomer matrix. PCMEC actuators have been demonstrated both in wet and dry environments.

Thermal expansion transducers will follow the same principle without phase change, using this time the expansion characterized by the coefficient of thermal expansion (CTE) of a material (Figure 6c). Tian et al. [141] reviewed soft electrothermal actuators principles, capabilities, and applications [141]. The thermal expansion of various materials can be used. Among them, elastomers [37-39], resins [40,41] or other polymers [43] have been reported. Paper is commonly used to add asymmetry to the design [43,44] (Section 2.4). Alternatively, SMP materials, detailed in Section 2.3.1, can be used as thermally expansive materials [42]. SMP-based actuators are among the most promising materials for soft robotics application, where the materials' thermal characteristics (glass temperature, temperature of crystallization, melting temperature and temperature of degradation) are of fundamental importance in reshaping of 
the devices as well as in the process of thermal actuation and activation. In fact, it is their ability to memorize an initial shape, to acquire a temporary one upon heating and to revert to the permanent shape trough thermal expansion that plays a key role in the actuation process. Even more, the degree of thermal expansion in polymer composites can be easily tuned by the incorporation of micro- and nanofillers, such as organoclay, carbon nanofiber (CNF), silicon carbide (SiC) and carbon black (CB) [45]. In such a way, the incorporation of nanofillers not only impacted the thermal properties of the materials but also enhanced their physicomechanical properties. Thermal expansion transducers are made of simple structures, since they rely on a single material expansion and the heat can be generated in various (remote or tethered) ways, further described in Section 2.3.5. Their capabilities is strongly related to the expansive material properties, in particular their CTE. They have been demonstrated at a very small scale and are able to achieve high curvatures with both low [40] and high [41] stiffness materials (Section 2.5).

Finally, swelling or drying transducers rely on the fluid absorption of a matrix (Figure 6d). Polymer materials are of particular interest in this section for their possibility to generate complex deployable structures with a great range of functionalities. The polymer actuators are known for their reversible shape and size change in response to various stimuli. In addition, the polymer systems are able to recover their initial shape by simple immersion in appropriate solvents, which is uncommon for a great range of actuators. Solvent-triggered SMP systems such as hyperbranched polyurethane (PUR) [52], polystyrene responsive to dimethylformamide [53] and toluene [54], or self-healed ethyl acetate-responsive poly(vinyl butyral) networks [55] are already described in the literature. Of particular interest are the water-responsive polymers based on cross-linked poly(vinyl alcohol) (PVA) [60], composites such as PVA/graphene oxide [61] or cellulose nanocrystal-loaded PUR elastomers [62]. Some solvent vapor-responsive materials were synthesized from natural rubber [63] or based on porous membranes [64]. Other works have demonstrated swelling with various responsive polymers [58,59]. Despite recent progress in the field, often, multi-step procedures need to be accomplished to achieve solvent-responsiveness, and this is only in "one-way" (temporary to permanent shape recovery), mainly due of the lack of architectural anisotropy within the material. The anisotropy can be approached with bilayer-type structures, further discussed in Section 2.4.1. Alternatively, Guo et al. [51] demonstrated swelling LCN structures, embedding the anisotropy in the material itself [51]. We have previously demonstrated that it was possible to fabricate bilayer solvent-sensitive polymeric actuators by one-step extrusion synthesis of cross-linked PCL (thermo-reversible Diels-Alder reactions between furan- and maleimide-modified PCL oligomers). The anisotropy in the bilayer was generated by incorporation of electrically conductive Multi-Walled Carbon Nanotubes (MWCNTs) in one of the layers. This resulted in differences in the layers' thermomechanical, swelling and actuating properties upon direct immersion or solvent vapor exposure [56]. The PCL actuators were defined as suitable for soft robotics: grabbing and transportation of objects, solvent vapor sensors and self-deployable structures. On the softest side of the materials, hydrogels can present a high swelling ratio, and their swelling is triggered by various stimulus or signals (DNA [46], heat [49], or pH [47]). Koetting et al. [142] proposed a complete review on swelling hydrogels [142], and Li et al. [36] focused on their photo-responsiveness [36]. As a general remark and comparing to other transducer principles (Section 2.5), swelling-based actuators allow for the generation of high strains and use with a large range of material stiffnesses and dimensions. However, its actuation time remains intrinsically barely explored.

\subsubsection{Other Mechanical Transducers}

Other types of transducers are not described here, either because they are not used to generating complex movements or are not stimuli-responsive or because they are not as flexible. In particular, shape memory alloys (SMA) have been widely studied and characterized. The authors redirect the reader to Mohd Jani et al. [143] for a complete review on SMA and their applications [143]. Even if well-known prior to the emergence of soft robotics, SMA transducers have recently been proposed for compliant systems and have-among others-been shown as promising for biomedical 
applications $[144,145]$. Compared to the other transducers reviewed in this work, SMAs are positioned in the high stiffness (28 GPa-82 GPa), high actuation stress (400 MPa-700 MPa) and low actuation strain (max 0.8) ranges [143]. Piezoelectric materials are also widely used as transducers [146]. In soft and continuum robotics, several works developed actuators based on pull wires/tendon [147-149] or concentric tubes [150]. Qiu et al. [151] proposed an untethered rigid robot driven by accoustic waves [151]. Crystal phase transition can also be used as a transducing principle. Taniguchi et al. [152] demonstrated walking and rolling of azobenzene crystals [152]. Finally, organic stimuli-responsive materials have recently emerged as a very innovative solution for integrated soft robotics [153,154]. Recently, McCracken et al. [100] proposed a comprehensive review on stimuli-responsive materials used as actuators, not limited to soft robotics [100].

\subsubsection{Stiffness Variation Transducers}

Several transducer materials or systems are also commonly used to allow stiffness variation. They do not generate deformation but a change of stiffness under a given stimulus. Several solutions have been reported in the reviewed papers. Blanc et al. [155] presented a classification of stiffness variation solutions for flexible medical devices. For exhaustive reviews on variable stiffness principles, the authors redirect the reader to [4,155-158]. Among the stiffness variation solutions, Low Melting Point Alloys (LMPA) use the liquid-solid phase transitions of metals or alloys to present extensive stiffness changes [155]. Most of the reviewed works use Cerrolow 117 or equivalent commercial materials $[75,159]$, with melting at $47^{\circ} \mathrm{C}$. The stiffness ranges from below $1 \mathrm{MPa}$ to above $1 \mathrm{GPa}[75,155]$, and the actuation time is generally several seconds, limited by the thermodynamics. LMPA embedded in soft robots must be encapsulated to avoid leakage at liquid state. Similarly, SMP can be used as variable stiffness solutions $[25,68,74]$, using the glass temperature transition of the polymer when heated. Zhang et al. [25] used VeroClear material as an SMP and reported a stiffness ranging between $9 \mathrm{MPa}$ and $1.4 \mathrm{GPa}$ around $70^{\circ} \mathrm{C}$. Ze et al. [74] used a custom SMP with stiffness varying between $2.4 \mathrm{MPa}$ and $2.9 \mathrm{GPa}$ in a similar temperature range. Finally, granular or layer jamming is another common tunable stiffness solution [12,32]. When a vacuum is applied on a granular medium or on a stack of layers (e.g., paper [32]), the friction forces between the grains/layers increase, leading to a higher stiffness. This solution presents the advantage of being fast, but the stiffness change is generally more limited compared to material-based solutions [155].

\subsubsection{Nonmechanical Transducers}

Additionally, other types of nonmechanical transducers are commonly used in soft robotics. We define nonmechanical transducers as materials or systems that can transform one stimulus into another, allowing to overcome transducers or stimulus limitations. The most common example is the use of the Joule effect responsible for local heat generation. In the literature, a significant number of fillers have been reported as active agents, such as nichrome $(\mathrm{NiCr})$ wires [34,159], resistive ink [25], copper wires [75,79,83], silver [93], silver nanoparticle coating [44], silver nanowires [39], gold [41], carbon nanotubes [37,42] or heating fabric [160]. Similarly, carbon black [35] or expanded intercalated graphite [161] can be embedded into an elastomer matrix to create a conductive heating composite material.

An important part of nonmechanical transducers is the use of photothermal effects. They convert light energy into heat by the transducer material itself. In particular, light-triggered SMPs deserve special attention due to their possibility to be activated in a remote way by high spatial and temporal resolutions (use of a large spectrum from UV-Vis to IR). In this aspect, of great interest are the plasmonic nanostructures derived from noble metals (silver (Ag) and gold (Au) NPs, nanorods, nanowhiskers or nanowires, and others) known to be highly effective in low amounts and characterized with large specific surface area and relatively strong surface plasmonic resonance $[118,162]$. A key element here is the nanofiller property to absorb a broad spectrum of wavelengths and to convert the light energy into heat (photothermal effect) $[163,164]$. For example, Ag nanowiskers can be used in a cross-linked 
polyacrylate layer for the fabrication of flexible and transparent SMP electrodes (light-emitting diode application) [54,165]. The design of bi- and multilayers resulted in flexible and fast electroresponsive PUR with shape recovery through the Joule effect $[165,166]$. A series of interesting studies have been published on light-triggered SMP applications: devices with visible and near-IR imaging sensitivity for soft tissue optical visualization [167], dynamic cross-linked PUR based on thermo-reversible chemistry (Diels-Alder reactions) containing polydopamine particles with self-healing properties [168] or poly(ethylene glycol)-based Au nanorods with induced healable properties [169], polymer composites with conferred stimuli-responsiveness to light illumination sequences for improved spatiotemporal shape control [170], electrically active polymer systems with incorporated carbon nanotubes (CNT) or graphene nanoplatelets [171] or poly(vinyl alcohol)-based films demonstrated self-cleaning surfaces upon polarized light [172]. In our previous study, we fabricated light-responsive PCL-based nanocomposites by combining two nanofillers (plasmonic AgNPs and cellulose nanocrystals) for successful photothermal effect upon IR illumination [66]. The materials revealed tunable contraction or bending movement in a remote way for potential application for object transportation and biomaterials (self-tightening knot for suture enclosure) with antibacterial properties. The combination of AgNP-decorated graphene oxide assembly grafted onto carbon fibers was used in SMP epoxy-based thermoset resins [173], and a synergistic effect between AgNPs-decorated graphene oxide fillers allowed material electrical conductivity [174].

Alternatively, Ze et al. [74] used a high-frequency alternating magnetic field to generate heat into a material doped with $\mathrm{Fe}_{3} \mathrm{O}_{4}$ particles [74]. As described above, Matsuoka et al. [24] and Han et al. [16] used the phase change of a fluid to generate pressure in fluidic chambers [16,24].

\subsection{Extrinsic Asymmetry}

To guide the transducer deformation into the desired kinematics, mechanical constraints can be added, called hereafter extrinsic asymmetry. This additional asymmetry is optional in the cases of anisotropic stimulus. This extrinsic asymmetry can be classified as passive or active (Table 2 and Figure 7). Here, we define passive asymmetry as a mechanical constraint that does not rely on any stimulation. On the other hand, active asymmetry is controllable and triggered by an additional stimulus. In practice, it consists in the integration of stiffness variation transducers in the actuator. This section describes the principal types of asymmetries. It does not aims to be exhaustive but to highlight the main principles reported. The principles described here are not exclusive and are compared quantitatively in Section 2.5.

Table 2. Classification of the different types of asymmetry.

\begin{tabular}{|c|c|c|c|}
\hline \multicolumn{2}{|c|}{ Classification } & Description & Examples \\
\hline \multicolumn{4}{|l|}{ None } \\
\hline \multirow{3}{*}{ Passive } & Multi-material & $\begin{array}{l}\text { Creating/increasing anisotropy } \\
\text { by using material with } \\
\text { different properties }\end{array}$ & $\begin{array}{l}\text { Bilayers }[10,19,26,37-44,46,49,56,57,59,70,175,176] \\
\text { Strain limiting fibres }[6,14,19,31,177] \\
\text { Reinforcement }[15,21,22,178-180] \\
\text { Kirigami }[8,11]\end{array}$ \\
\hline & Geometry & $\begin{array}{l}\text { Creating/increasing anisotropy } \\
\text { by changing the geometrical } \\
\text { properties of a single material }\end{array}$ & $\begin{array}{l}\text { Patterns }[50,77,80,81] \\
\text { Eccentricity[7,16,18,20,23,79,90,92] } \\
\text { Swelling rate gradient }[49] \\
\text { Bellows }[9,24,28-30]\end{array}$ \\
\hline & $\begin{array}{l}\text { Material } \\
\text { customization }\end{array}$ & $\begin{array}{l}\text { Creating/increasing anisotropy } \\
\text { by customizing the material } \\
\text { properties locally }\end{array}$ & $\begin{array}{l}\text { Prestrain }[65,69,78] \\
\text { Magnetic profile [71-73,123] } \\
\text { Gradient [47-49] } \\
\text { Anisotropic stiffness [89] } \\
\text { Local fibre alignment }[51,58,84]\end{array}$ \\
\hline Active & $\begin{array}{l}\text { Stiffness } \\
\text { variation }\end{array}$ & $\begin{array}{l}\text { Creating/increasing anisotropy } \\
\text { by controlling the stiffness of on } \\
\text { part of the structure }\end{array}$ & {$[12,25,32,68,74,75,159]$} \\
\hline
\end{tabular}




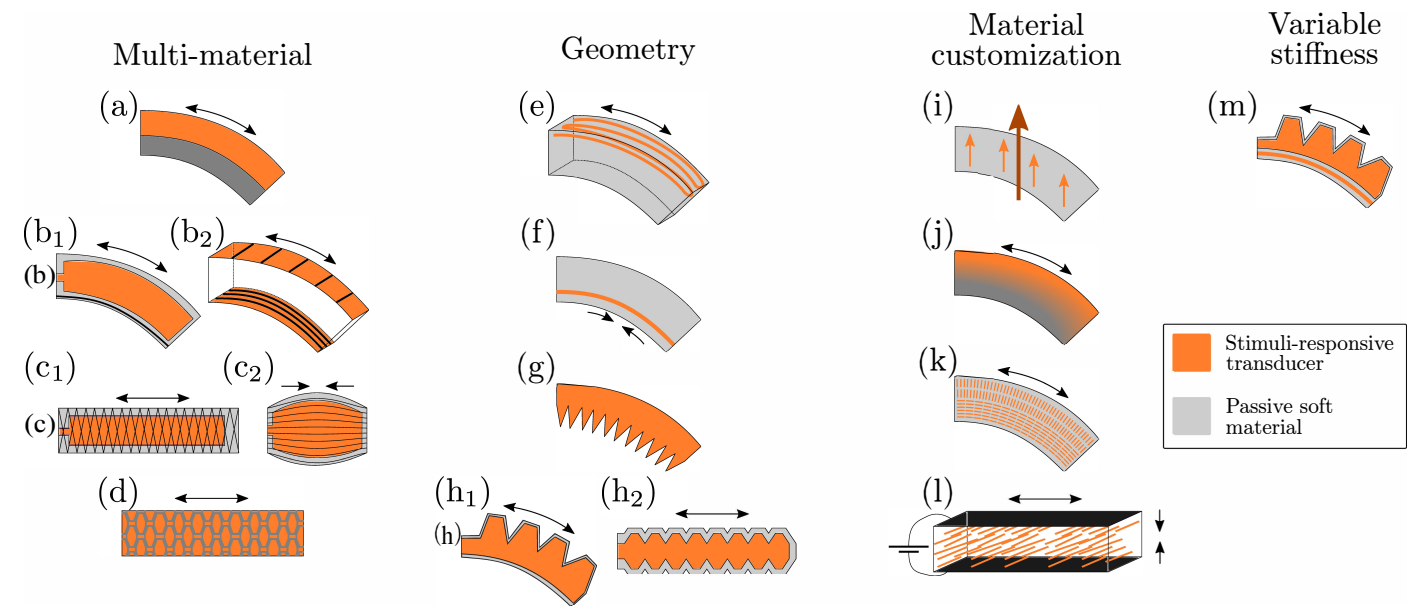

Figure 7. Typical examples of the different types of extrinsic asymmetry: most of the actuators are combinations of the nonexhaustive list of principles illustrated here. (a) Bilayer, (b) limiting fibre/layer: $\left(\mathbf{b}_{1}\right)$ acting as neutral fibre or $\left(\mathbf{b}_{2}\right)$ forming a pattern, $(\mathbf{c})$ reinforcement: $\left(\mathbf{c}_{1}\right)$ fibre reinforcement and $\left(\mathbf{c}_{2}\right)$ McKibben muscles, (d) Kirigami reinforcement, (e) expansive/contractive pattern, (f) eccentric actuation, $(\mathbf{g})$ swelling rate gradient, $(\mathbf{h})$ bellows: $\left(\mathbf{h}_{1}\right)$ asymmetrical or $\left(\mathbf{h}_{2}\right)$ symmetrical, $(\mathbf{i})$ magnetization profile, (j) gradient, (k) local fibre alignment, (l) anisotropic stiffness and (m) stiffness variation.

It is important to note that, if asymmetry is introduced using an anisotropic stimulus and/or transducer, no extrinsic asymmetry is required. This is typically the case with SMP [66]. LC deformation can be guided using localized laser stimulus [85] or structured light [82]. Finally, IMPC intrinsic anisotropy can be used as is [95]. However, most of the recently reported actuators combine several sources of anisotropy in their design, even by adding extrinsic asymmetry to anisotropic transducers or stimulus or by multiplying the types of extrinsic asymmetries. This allows for finer guidance of material deformation into the desired kinematics and/or for optimization of the mechanical outputs of the actuator.

\subsubsection{Multi-Material Asymmetry}

Among passive asymmetry strategies, the multi-material solution is the most common solution. It relies on asymmetry between two or more materials. This asymmetry can be expressed in the rigidity of the material and in the stimuli-responsiveness. Figure 7a illustrates the well-known principle of the bilayer. In this example, bending is generated by the expansion of the top layer relative to the bottom layer. As stated before, the bottom layer can be purely passive or presents a smaller expansion than the active layer. The role of the passive layer is to act as a neutral fibre, i.e., to have a high longitudinal stiffness, while having a negligible bending stiffness. Yao et al. [39] developed bilayers made of an expansive Polydimethylsiloxane (PDMS) and a passive polyimide layer, actuated by a flexible heater, and demonstrated the possibility to vary locally the actuator curvature by modifying the limiting layer height (and consequently structural stiffness) or the heating intensity [39]. Similarly, Hu et al. [44] developed a bilayer paper-elastomer, completed by a flexible heating layer. In this case, the paper can even act as a passive layer or as an active layer by swelling when subjected to moisture. If the passive material presents anisotropy, the bilayer configuration can be used to generate twisting by arranging its stiffer direction with an angle between $0^{\circ}$ and $90^{\circ}$ relative to the actuator axis [19,44].

Similar to the bilayer, a limiting fibre can be embedded in the active structure. The strain limiting fibre cannot be elongated, while the active material expands. The limiting fibre can be unique (Figure $7 b_{1}$ ), acting as neutral fibre [6], or can form a pattern (Figure $7 b_{2}$ ) [177]. As for the bilayer, this pattern angle can be modified to generate helixing or twisting [177].

Reinforcement typically of double helix of fibre prevents radial expansion while having a negligible axial and bending stiffness and guides an isotropic deformation into elongation (Figure $7 \mathrm{c}_{1}$ ). It is commonly used in combination with a limiting fibre to generate bending. It can also be used 
to generate other kinematics by varying the reinforcement pattern, such as twisting (using a single helix) [178]. For pneumatic actuators, a sufficiently low pitch allows for optimization of the mechanical output of the actuator [181]. It is also commonly used to generate (Figure $7 c_{2}$ ) contraction, in so-called McKibben muscles [21]. Using similar principles, origami reinforcement, based on folded structures, has also been demonstrated [180].

Kirigami reinforcement, using cuts in a thin layer wrapped around the transducer, can be used as an alternative for fibre reinforcement, allowing local control of the reinforcement properties by varying the cutting pattern (Figure 7d). Rafsanjani et al. [11] demonstrated elongating pneumatic robots with asymmetric frictional properties [11]. Twisting and bending have also been demonstrated [8].

\subsubsection{Geometry}

Alternatively, the asymmetry can be introduced in the geometry. This type of asymmetry is rarely used alone but is very common. Expansive material patterns can be used to generate an asymmetric stiffness (Figure 7e) and to direct the deformation. When the active material expands, the expansion can be isotropic or anisotropic locally but will only be transmitted along the matter direction. Globally, elongation is then larger in the direction of the long fibres [50]. In the case of contractile fibres, bending occurs in the other direction [80]. As previously noted, twisting can be obtained by varying the pattern direction [50]. This type of asymmetry has been demonstrated with LCE [77,80,81] and swelling [50] but could theoretically be expanded to any expansive transducer.

In the eccentric actuation configuration illustrated in Figure $7 f$, the transducer material contraction induces bending because of its eccentricity relative to its center (neutral fibre). This principle has been exploited using McKibben muscles placed longitudinally around a soft structure [23]. Placing additional muscles in a helix can lead to generation of twisting and combinations of basic kinematics [20]. Sun et al. [92] used the same principle but with TCP [79] and DEA [79]. Similarly, bending can be obtained by buckling of eccentrically placed DEA [90]. If the transducer elongates, bending will occur in the opposite direction. This is commonly used with fluidic chambers with one [18] or multiple chambers to bend in every direction $[7,12,16]$.

The swelling rate gradient can also be customized to obtain a desired deformation and has been demonstrated with hydrogel (Figure 7g) [49]. The swelling is a function of the surface/volume ratio; by varying this ratio across the actuator height, the swelling rate can be controlled locally. The bending will be generated towards the regions of minimal swelling. This effect is reinforced by the teeth structure, which induces a longitudinal stiffness difference with the plain side.

Bellow structures are another well studied class of actuators, mainly used with fluidic chambers. If the bellows are asymmetrical (Figure $7 \mathrm{~h}_{1}$ ), as in commonly called PneuNets actuators, bending is obtained $[9,24,159]$. This asymmetry is commonly used in combination with a neutral fibre to optimize the output mechanical work [28]. Helixing can also be generated by changing the bellow angle [28]. If the transducer expands (Figure $\left.7 \mathrm{~h}_{2}\right)$, the bellow geometry will generate an elongation of the actuator [31]. Inversely, contraction will be obtained with negative pressures [31]. Other bellow-like structures can be used to vary the stiffness along the actuator height. As an example, already in 1996, Lazeroms et al. [182] proposed a design with cuts on one side of a reinforcing tube around a fluidic chamber, decreasing the rigidity of this side and guiding the deformation into a flexion towards the other side [182].

\subsubsection{Material Customization}

Finally, the extrinsic asymmetry can be introduced in the transducer material itself, what we call here material customization. This asymmetry is not strictly speaking extrinsic but is controlled during transducer fabrication or at the material level. This type of asymmetry is less generalizable because it is more dependent on transducer intrinsic properties or anisotropy. In the case of magnetic actuators, the magnetization profile can be customized locally (Figure 7i) by changing the magnetic particle 
orientations in the soft matrix. The dipoles will align when a magnetic field is applied, allowing for generation of various kinematics [71,72,123].

Similar to the bilayer configuration, transducer properties can be varied locally to generate stimuli-responsiveness or stiffness gradient (Figure 7j). This has been demonstrated with swelling gradients, varying the material composition or the cross-linking density across the transducer height [49]. When stimulated, bending is observed towards the less expansive side. This class of asymmetry allows for very fine control of the local transducer properties and can be declined to generate various kinematics $[47,48]$. Using a prestrain gradient to direct the deformation has also been studied in the case of SMP $[65,69]$.

With anisotropic transducers, local fibre alignment can be achieved to guide the deformation (Figure 7k). This has been demonstrated with LC. The material contracts in the direction of the fibres. By changing the fibre orientation locally, the macroscopic contraction direction can be controlled. The fibre alignment is generally set during fabrication $[51,81,84]$. Alternatively, the fibre direction can be modified by prestraining LC mateirals [83].

In the same way, anisotropic materials can be used passively to control the local anisotropic stiffness of the actuator. In the example illustrated in Figure 7l, LC anisotropic material is used as a passive dielectric layer in a DEA to guide elongation along the length of the actuator [89].

\subsubsection{Stiffness Variation}

Considering active asymmetry, the most common method (and the only one identified in this review) consists in the use of stiffness variation (LMPA or SMP) to customize the deformation properties of the actuator. As illustrated in Figure $7 \mathrm{~m}$, a variable stiffness layer can be added to control the actuation behaviour of a transducer and to lock it in the actuated configuration [25] (orange layer in the bottom of the schematic). A similar principle can be applied for the other basic kinematics [32]. Variable stiffness can be used to control the actuator kinematics by reducing the stiffness of a given point or segment of an actuator, which will consequently deform under the transducer action $[75,159]$. Variable stiffness solutions have been widely used with fluidic chamber transducers but were also demonstrated with magnetic actuators [68,74]. Active asymmetry using stiffness variation is a very promising solution towards the development of soft robots able to achieve complex tasks. It allows for reprogramming of the actuator kinematics in real time or at least has several actuation modes and controls its structural rigidity.

\subsection{Overview and Quantitative Comparison of the Transducers}

Even if strongly related to the transducer (energy conversion efficiency, scaling laws, material stiffness and transducing speed), the actuators' mechanical outputs (stress, strain and curvature) depend also on the stimulus intensity and the extrinsic asymmetry (optimization of a given kinematics). The capabilities and properties of the reviewed actuators are then compared for the different transducers in Figure 8 and as a function of the asymmetry principles in Figure 9. The references of the works compared to build those figures can be found in Appendix A. When relevant, the actuators reviewed in this work are compared with muscles [143,183]. 
(a)

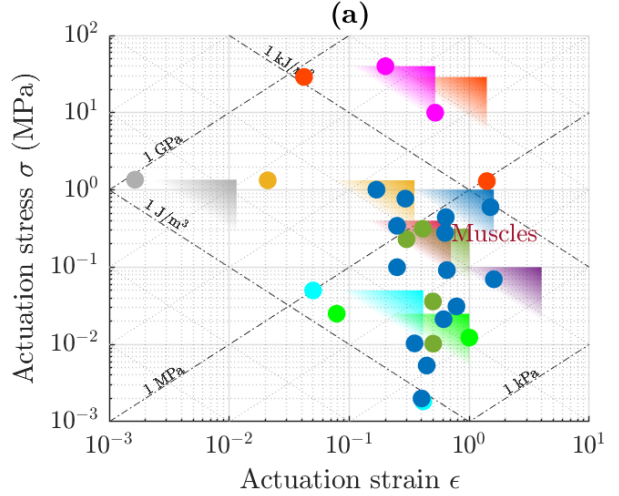

(c)

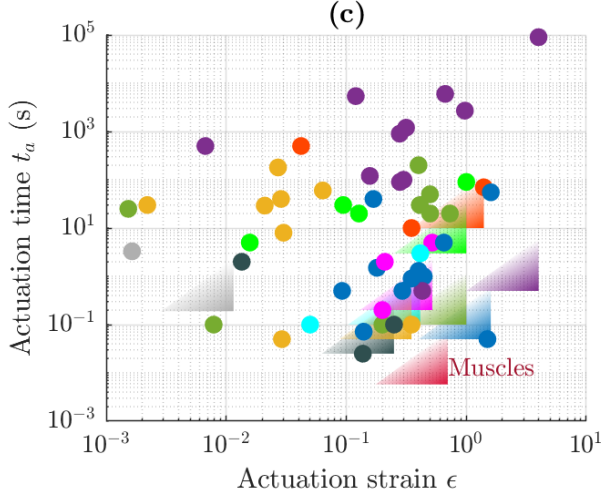

(b)

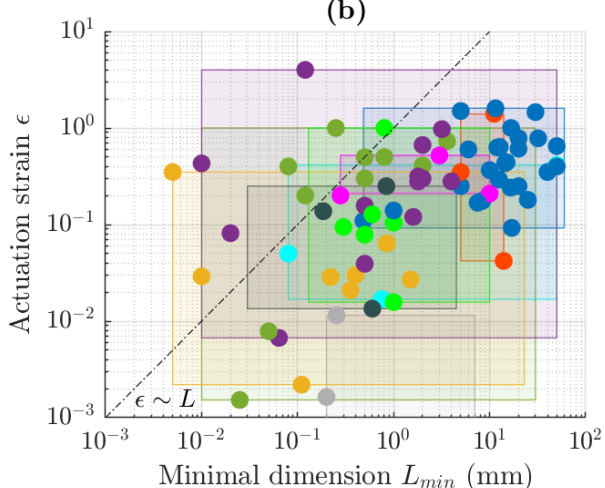

(d)

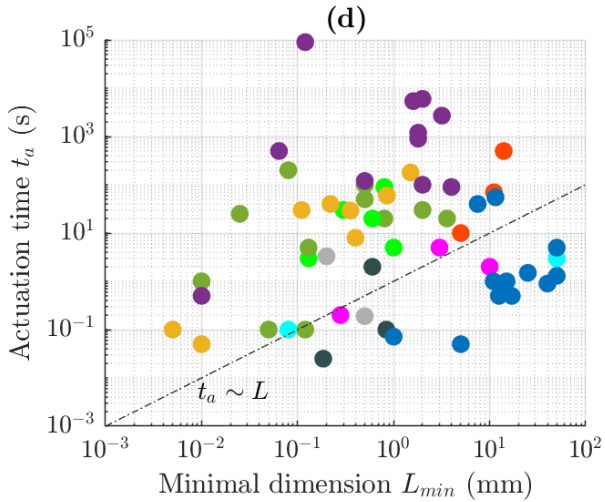

(e)

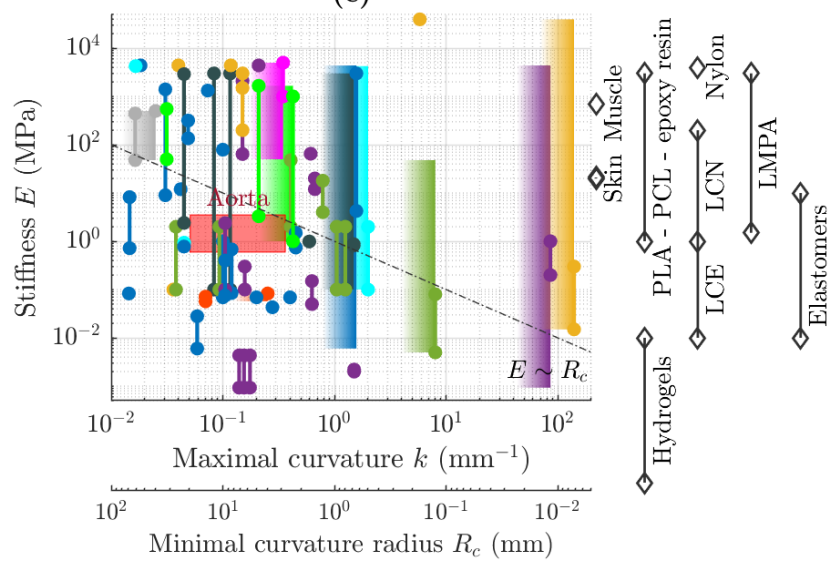

- DEA

- Fluidic chamber

- IPMC

- LC

- Magnetization

- PCMEC

- SMP

- Swelling

- TCP

- Thermal expansion

Figure 8. Comparison of the different actuators: each dot or line represents one actuator, and each color is a type of transducer. The triangles or zones vertical and horizontal borders represent the maximal or minimal values reported for a given transducer. (a) Actuation stress as a function of the actuation strain. The diagonal lines of positive slope represent a constant value of $\sigma / \epsilon$, which is a characterization of the stiffness of the actuator. The diagonal lines of negative slope $\sigma \epsilon$ are lines of constant energy density. (b) Actuation strain as a function of the minimal dimension. The dashed line has a slope of $1(\epsilon \sim L)$. (c) Actuation time as a function of the actuation strain. (d) Actuation time as a function of the minimal dimension. The dashed line has a slope of $1\left(t_{a} \sim L\right)$. (e) Stiffness as a function of the curvature. The stiffness corresponds to the stiffness of the main actuator material. The right side of the zones represents the maximal curvature reported for a class of actuator, while their height corresponds to the range of stiffness reported. Typical material stiffnesses are reported on the right side of the graph. The dashed line has a slope of $-1\left(E \sim R_{c} \sim 1 / k\right)$. See Figure A1 for references. 
(a)

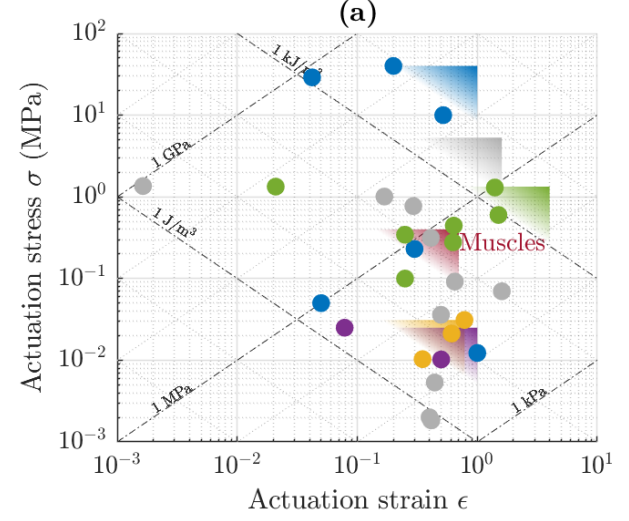

(b)

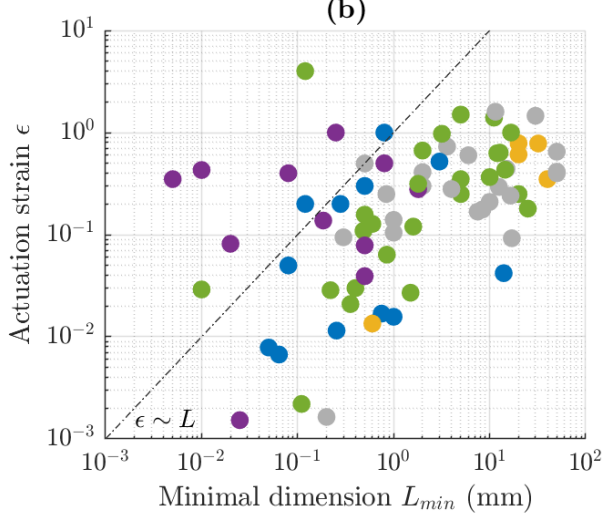

(c)

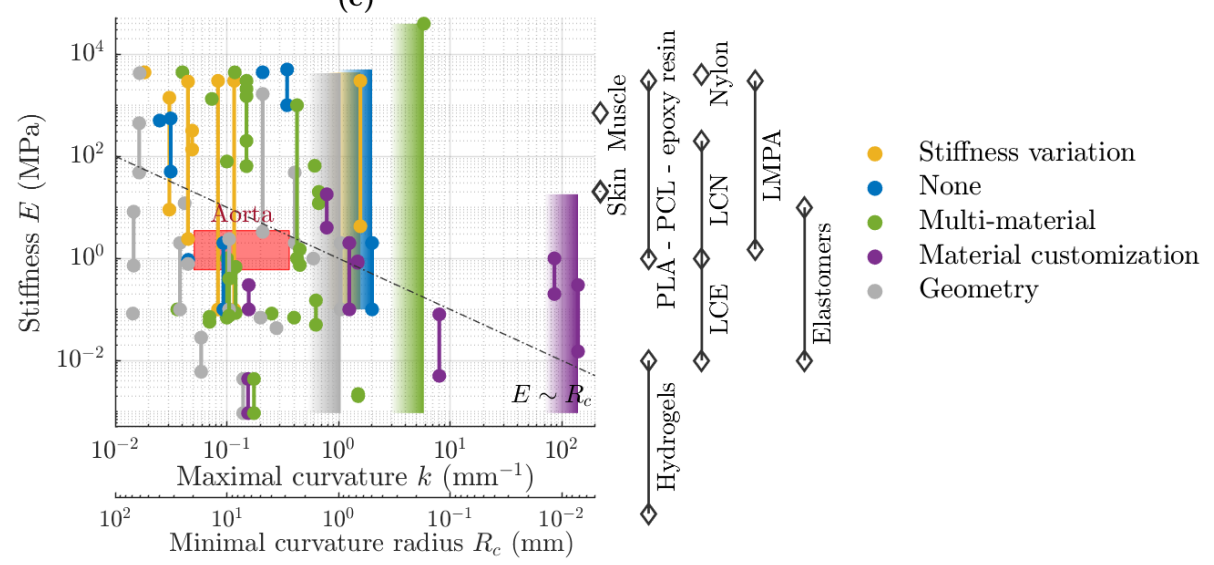

Figure 9. Comparison of the different types of extrinsic asymmetries: each dot or line represents one actuator, and each color is a type of asymmetry. The triangles or zones vertical and horizontal borders represent the maximal or minimal values reported for a given transducer. (a) Actuation stress as a function of the actuation strain. The diagonal lines of positive slope represent a constant value of $\sigma / \epsilon$, which is a characterization of the stiffness of the actuator. The diagonal lines of negative slope $\sigma \epsilon$ are lines of constant energy density. (b) Actuation strain as a function of the minimal dimension. The dashed line has a slope of $1(\epsilon \sim L)$. (c) Stiffness as a function of the curvature. The stiffness corresponds to the stiffness of the main actuator material. The right side of the zones represents the maximal curvature reported for a class of actuator, while their height corresponds to the range of stiffness reported. Typical materials stiffness are reported on the right side of the graph. The dashed line has a slope of $-1\left(E \sim R_{c} \sim 1 / k\right)$. See Figure A1 for references.

The stress-strain diagrams presented in Figures $8 \mathrm{a}$ and $9 \mathrm{a}$ are a typical representation of actuators proposed by Huber et al. [183]. It allows for a rapid overview of the mechanical capabilities of the actuators. In logarithmic axes, the diagonal lines of the positive slope represent constant values of $\sigma / \epsilon$, which is a characterization of the stiffness of a given actuator. The diagonal lines of negative slope $\sigma \epsilon$ are the lines of constant energy density. Here, the coordinates of each actuator correspond to the maximal stress and strain reported. In reality, the actuator will not be able to produce a maximal force and deformation at the same time, and the energy density of each point is overestimated. Similarly, the maximal values (triangles) for each type of transducer and asymmetry principle are overestimated. However, it allows to give an order of magnitude of these characteristics to compare the different solutions. When not reported, the stress and strain as well as the curvature (Figures $8 \mathrm{e}$ and 9c) have been computed as described in Section 2.1. Note that the stress and strain (or force and displacement) of a given actuator are rarely both reported. It can be noted that all classes of soft transducers, except IPMC, develop strains in a similar range. However, as the IPMC dimensions are generally small, satisfying curvatures can be obtained (Figure 8e). A similar observation can be made 
concerning asymmetry principles. It is still interesting to note that higher strains and curvatures can be obtained from multi-material asymmetry than from geometrical asymmetry (Figure 9a). In case of bending actuators, these quantities will be maximized with the asymmetry, that can be higher with multi-material configurations. Note that no quantity exists to characterize the anisotropy of a solution. However, its quantification can be approached by the mechanical capabilities of the final actuator. A wide range of maximal actuation stress is reached by the different transducers. The higher values were reported using TCP [91,93] and wax-based PCEMC [35]. As stated before, the energy density of a given actuator depends among others on the stimulus intensity and the transducers' energy conversion efficiency. Note that real energy densities and efficiencies are rarely reported, making the comparison difficult.

Figures $8 \mathrm{~b}$ and $9 \mathrm{~b}$ allow a comparison of the most reported quantifiers of actuators, the actuation strain and the minimal actuator dimension. Even if the strain does not depend on the actuator dimension, a correlation between both quantities can be observed. At a smaller dimension, a smaller strain is required to develop a given curvature. The actuators above the line of slope one $(\epsilon \sim L)$ develop a higher strain for their size. Clearer zones appear here for the different transducers (Figure $8 \mathrm{~b}$ ). It is interesting to note that swelling, LC and thermal actuators develop high strains for their dimensions. This is also observed in Figure 8e, where they develop the highest curvatures. Moreover, they also cover the largest zones in terms of achieved dimensions, while PCMEC, TCP and fluidic actuators remain mostly used at the submillimetric scale. Comparing the asymmetry principle, it can be observed that material customization is widely used at a small scale and for actuators above the line of slope 1, in particular because of the compatibility of this fabrication method with the corresponding transducers. On the other side, stiffness variation and geometric asymmetry solutions have been mostly reported on a larger scale.

Figure $8 \mathrm{c}, \mathrm{d}$ introduces the actuation time, which seems independent on the asymmetry principle. It mainly depends on the stimulus-transducer couple and on the actuator size. As a general observation, IPMC, thermal (SMP, thermal expansion, PCMEC and LC) and swelling transducers are slow, limited by the actuation principle. However, small actuation times have been demonstrated for each transducer using various strategies. In particular, for all the transducers, a relation is observed between the actuators' actuation time and their dimensions (Figure $8 \mathrm{~d}$ ). On this representation, the actuators below the line of slope one $\left(t_{a} \sim L\right)$ develop a faster actuation for their size. As expected, TCP, fluidic chamber and magnetic actuators show the best capabilities, while swelling actuators are shown to be the slowest transducing principle at a given size. It is important to consider reversability of the actuation, not represented here. In particular, one-way SMPs need to be reprogrammed between actuation. Moreover, the transducers relying on thermal actuation (PCMEC, thermal expansion, LC and TCP) generally present a slower deactivation time than actuation time because of the cooling time. Similarly, chemically actuated transducers (swelling, LC and IPMC) can present highy asymmetric actuation rates depending on the chemical reactions involved. However, several proposition have been presented throughout Section 2.3 to overcome these limitations. Finally, the possibility to achieve multi-cycle actuation is a key feature of actuators. As discussed by Miriyev [184] and confirmed in this review, numerous studies only demonstrate one actuation cycle, even if more are possible [184].

Finally, Figures $8 \mathrm{e}$ and $9 \mathrm{c}$ represent the reviewed actuators as a function of their curvature and stiffness. The curvature is proportional to the dimensions of the actuators (Section 2.1). The stiffness corresponds to the main material modulus. A range is given for variable stiffness solutions and when the stiffness is estimated. The typical modulus range of the materials or classes of materials are given on the right side of the figure. The data are from Hines et al. [3] (muscle, skin, hydrogels, LCE and LCN [3]), Blanc [185] (LMPA, PLA, PCL and epoxy resin [185]) and from the current review (elastomers). The aorta data are from [186,187]. This representation allows us to compare actuators based on quantities typically defined by the application requirements. As an example, the typical stiffnesses and curvature of the aorta are represented as a zone. Actuators for minimally invasive surgery should be placed in a similar region of the graph. It can be observed that a large range 
of stiffness can be obtained with most of the transducers. Even if some extreme curvatures have been demonstrated [40,47], most of the works achieve curvatures between $0.01 \mathrm{~mm}^{-1}$ and $1 \mathrm{~mm}^{-1}$, corresponding to the radius of curvatures from $1 \mathrm{~mm}$ to $10 \mathrm{~cm}$. As expected from Figure 8a, fluidic chamber, thermal expansion and swelling transducers can reach a large range of stiffness (from less than $10 \mathrm{kPa}$ to more than $1 \mathrm{GPa}$ ). While LCs are intrinsically relatively soft, TCP, IMPC, and to a lesser extent SMP and DEA transducers are on the stiffer side of the range. Figure 9c highlights the solutions using stiffness variation. Material customization, as previously stated, seems to be the best solution to generate high curvatures and is generally used with low stiffness materials.

\section{Towards Complex Motions}

From the previously detailed basic kinematics and their combinations, soft robots capable of complex motions and of achieving complex tasks can be designed. Here, we define complex motions as deformations beyond pure elongation, shear, twist or bending. This deliberately broad term gathers a variety of motions enabled by the infinite number of degrees of freedom of continuous robots. Similarly, we define the complex motion generation strategy as the way used to create a complex motion from basic kinematics when building the actuator into the rest of the robot body. While the strategies to generate simple kinematics have been largely studied, the methods to generate or program complex motions remain mostly exploratory and lack of methodology. Here, we propose a classification of the strategies (Section 3.1) and of the complex motions (Section 3.2). These complex motions are strongly related to the targeted applications and will be discussed together. Figure 10 presents an overview of the different strategies to achieve complex motions based on simple kinematics. This representation aims to give an overview of the different strategies possible. It highlights that various motions can be obtained with most of the strategies. However, several strategies have been more investigated, and other less studied promising solutions could open new opportunities.

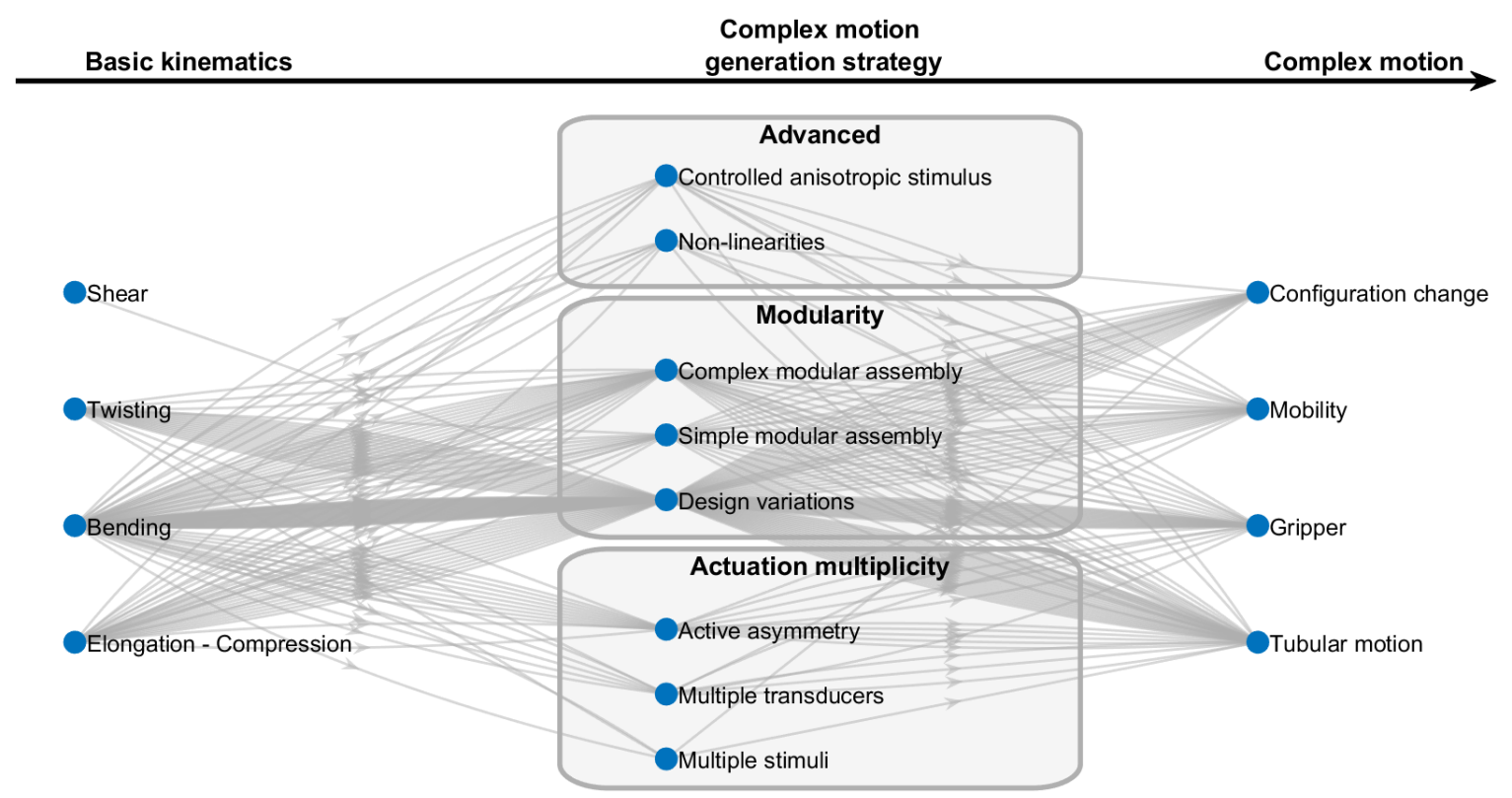

Figure 10. Roadmap towards complex motion generation: one soft robot is represented by a set of arrows. Complex motions can be generated using several strategies in parallel.

\subsection{Complex Motions Generation Strategies}

Towards many soft robotics applications, the ability of complex motion generation to achieve sophisticated tasks is a key point to unleash their potential. Various strategies have been recently proposed but are generally very exploratory and lack a real design methodology. Here, we proposed a classification based on three types of complex motions generation strategies: actuation multiplicity, 
modularity and advanced strategies (Table 3). As previously, these methods can be combined, and the choice of the strategy will be strongly dependent on the transducer and stimulus. It is important to notice that the generation of complex motions is not always explored, in particular when the application requires a simple kinematics or when the work focuses on the transducer capabilities. The versatility or reprogrammability of a given actuator, i.e., the eventual multiplicity of shapes and motions achieved, is an important comparison criteria here. This versatility can be embedded in the stimuli-responsiveness of the transducer itself, in the asymmetry (active asymmetry) or with other strategies developed in this section. Focusing on reconfigurable and shape changing robots, Shah et al. [188] proposes an overview of recent advances and further challenges of the field [188].

Table 3. Complex motion generation strategies classification.

\begin{tabular}{|c|c|c|c|}
\hline Classification & & Description & Examples \\
\hline None & & Simple kinematics only & {$[6,14,18,24,95,152]$} \\
\hline \multirow{3}{*}{$\begin{array}{l}\text { Actuation } \\
\text { multiplicity }\end{array}$} & Multiple stimuli & $\begin{array}{l}\text { Several stimuli per } \\
\text { transducers }\end{array}$ & {$[86,176,189]$} \\
\hline & $\begin{array}{l}\text { Multiple } \\
\text { transducers }\end{array}$ & $\begin{array}{l}\text { Several transducers per } \\
\text { actuator }\end{array}$ & {$[42,44,46,49,85]$} \\
\hline & $\begin{array}{l}\text { Active } \\
\text { asymmetry }\end{array}$ & Stiffness variation & {$[12,32,68,74,75,159,190]$} \\
\hline \multirow{3}{*}{ Modularity } & Design variations & $\begin{array}{l}\text { Modified actuator design } \\
\text { to achieve a given task }\end{array}$ & {$[8,15,37,39,48,51,58,71,77,81]$} \\
\hline & $\begin{array}{l}\text { Simple modular } \\
\text { assembly }\end{array}$ & $\begin{array}{l}\text { Reproduction of a given } \\
\text { module, controlling the } \\
\text { assembly with a single } \\
\text { stimulus }\end{array}$ & $\begin{array}{l}\text { Hinges }[41,57,80] \\
\text { Tubular segments }[9,40,178,179] \\
\text { Metamaterials }[59,71,81] \\
\text { Other [91] }\end{array}$ \\
\hline & $\begin{array}{l}\text { Complex } \\
\text { modular } \\
\text { assembly }\end{array}$ & $\begin{array}{l}\text { Reproduction of a given } \\
\text { module, controlling the } \\
\text { assembly with one } \\
\text { stimulus per module }\end{array}$ & $\begin{array}{l}\text { Tubular segments }[7,12,22,75,90] \\
\text { Voxels }[13,17,32,94] \\
\text { Hinges }[70] \\
\text { Rigid base }[28,30,79] \\
\text { Other [92] }\end{array}$ \\
\hline \multirow{2}{*}{ Advanced } & Non linearities & $\begin{array}{l}\text { Exploitation of a nonlinear } \\
\text { transducer response }\end{array}$ & {$[9,10,16,21,41,67]$} \\
\hline & $\begin{array}{l}\text { Controlled } \\
\text { anisotropic } \\
\text { stimulus }\end{array}$ & $\begin{array}{l}\text { Variable or structured } \\
\text { controlled stimulus }\end{array}$ & $\begin{array}{l}\text { Light [82] } \\
\text { Magnetic field }[59,72,75,123]\end{array}$ \\
\hline
\end{tabular}

\subsubsection{Actuation Multiplicity}

Several strategies can be classified as actuation multiplicity: some transducers show different actuation modes in response to multiple stimuli. Different kinematics will be obtained depending on the stimulus, and using these transducers properties allows for large versatility in the actuator capabilities. First, a given transducer can react to multiple stimuli. This solution depends on the stimuli-responsive behaviour of the transducer and remains widely unexplored. As shown in Figure 11a, Zhao et al. [176] used a supramolecular polymer in a bilayer configuration, responding both to UV light and humdity changes through incorporation of multiple stimuli-responsive groups [176]. Ryabchun et al. [86] integrated hydrazone photoswitches into LC, allowing stable reversible actuation using different light wavelengths [86]. Odent et al. [49] described a bilayer composed of two layers with different $\mathrm{pH}$ - and thermal-responsive swelling, allowing bidirectionality and fine control of the bending behaviour of the system [49]. 
(a)

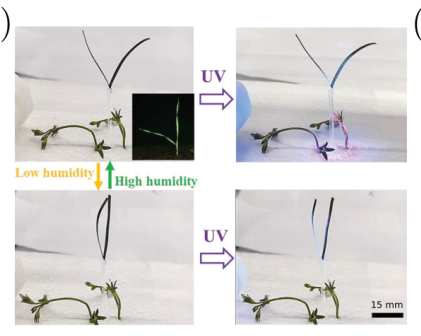

(d)

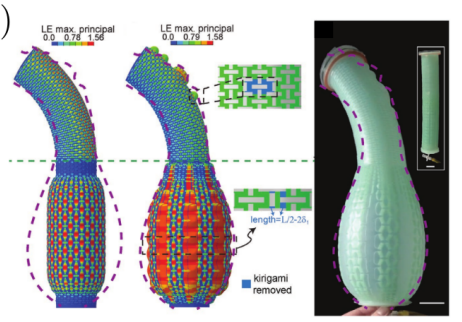

(g)

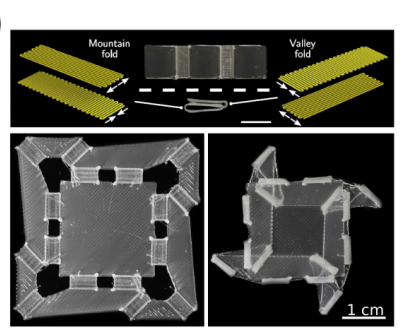

(k)

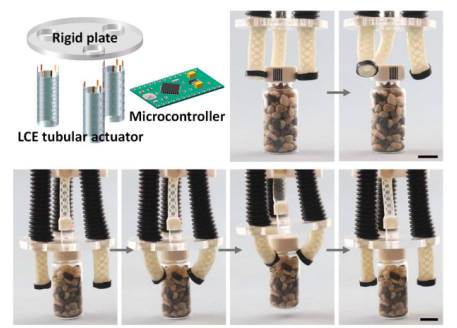

(m)

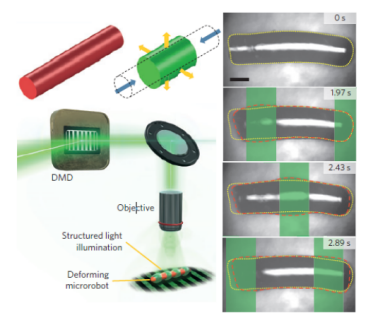

(b)

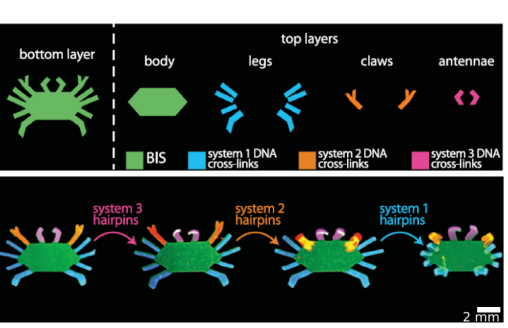

(e)

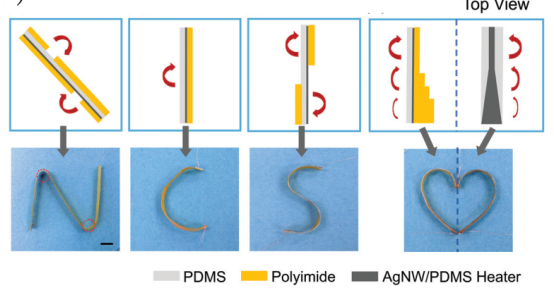

(i)

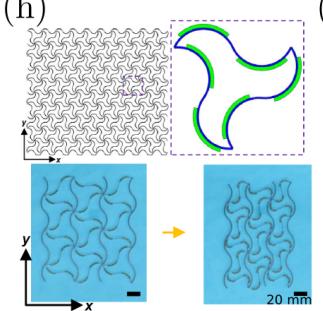

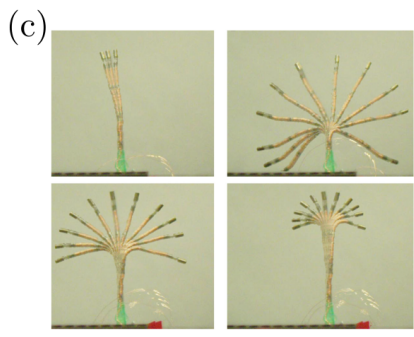

(f)

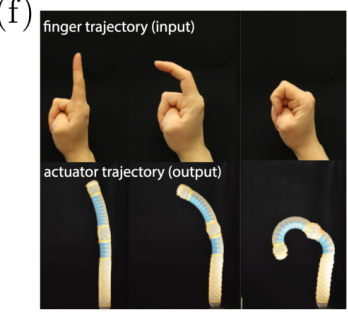

(j)

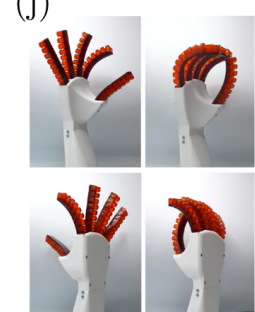

(1) 100
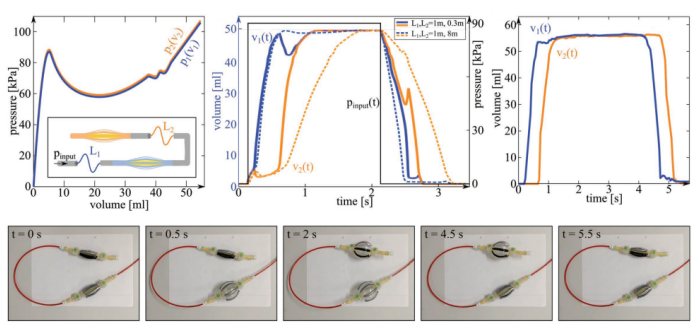

(n)

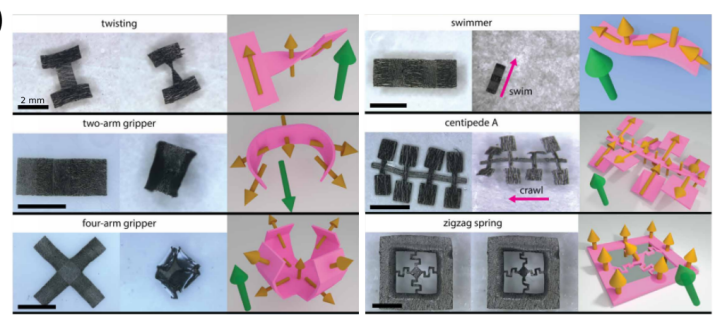

Figure 11. Examples of strategies towards the generation of complex motions. (a) Multiple stimuli [176]. Copyright 2019, John Wiley and Sons. (b) Multiple transducers [46]. Copyright 2017, American Association for the Advancement of Science. (c) Active asymmetry [75]. Copyright 2019, John Wiley and Sons. (d) Design variations (kirigami) [8]. Copyright 2018, John Wiley and Sons. (e) Design variations [39]. Copyright 2017, Royal Society of Chemistry. (f) Tubular segments simple modular assembly [178]. Copyright 2017, National Academy of Sciences. (g) Hinges simple modular assembly [80]. Copyright 2019, American Association for the Advancement of Science. (h) Metamaterial simple modular assembly [59]. Copyright 2018, American Association for the Advancement of Science. (i) Voxel complex modular assembly [32]. Copyright 2020, John Wiley and Sons. (j) Rigid base complex modular assembly [30]. Copyright 2020, MDPI. (k) Rigid base complex modular assembly [79]. Copyright 2019, American Association for the Advancement of Science. (1) Nonlinearities [21]. Copyright 2018, John Wiley and Sons. (m) Controlled anisotropic stimulus [82]. Copyright 2016, Springer Nature. (n) Design variation and controlled anisotropic stimulus [123]. Copyright 2018, American Association for the Advancement of Science. 
Similarly, several works have proposed to combine multiple transducers. These transducers can even react to different stimuli or at different levels of a single stimulus. This approach is similar to the multiple stimuli strategy but is more versatile since every transducer can be customized to react to a given stimulus. This strategy opens many possibilities in terms of actuation versatility; each transducer stimulation can lead to a given motion. Cangialosi et al. [46] demonstrated this principle with DNA-induced swelling (Figure 11b) [46], with different domains of the actuator responding selectively to different DNA sequences. Alternatively, bilayer mechanisms with two stimuli-responsive layers have been demonstrated, for example, with an hygroscopic expansive paper layer and a thermally expansive elastomer layer [44]. This configuration allows for bidirectional bending when heated or placed in a humid environment. Here, each layer acts passive when the other is stimulated. Similarly, Sachyani Keneth et al. [42] developed a bilayer-like actuator composed of a passive kapton layer and an SMP layer, additional to the heating layer [42]. This configuration combines the shape memory capacities and the thermal expansion of SMP, allowing to program unique shapes and motions. Lahikainen et al. [85] used multiple segments of LC [85], one segment actuated by photothermal effect with a first wavelength and one segment using photochemical actuation mode with two other wavelength having antagonist effects. This allows for a fine control of the motion using different stimulation patterns. Furthermore, the possibility to realize complex nonreciprocal motions while simplifying the actuation signal complexity has been demonstrated.

Finally, as discussed in Section 2.4.4, a particular class of transducer multiplicity consists in using active asymmetry, i.e., stiffness variation, to generate complex motions. The difference here is that the active asymmetry does not generate mechanical work but a change in the mechanical properties. Chautems et al. [75] demonstrated a magnetically actuated catheter with different variable stiffness segments that can be stimulated separately (Figure 11c) [75]. The same principle has been demonstrated with fluidic actuation [12]. As stated before, this principle has also been widely used to demonstrate shape locking [68,74]. The possibility to generate kinematics to change continuously a structure or voxel using local stiffness variation has also been demonstrated $[32,159,190]$. This particular solution seems especially promising to generate extremely versatile soft robots.

\subsubsection{Modularity}

A second class of strategies is based on actuation modularity. The most common consists in design variations. The basic actuator is modified to achieve the targeted task. Variable bilayer configuration or magnetization profiles are classical examples. Note that almost all actuators use combinations of design variation with other strategies to generate complex motions. The motions achieved using this strategy can be extremely complex but cannot be modified, since they are programmed in the kinematics. As a typical example, Sun et al. [15] modified the reinforcement pattern of tubular pneumatic actuators to generate various motions [15]. Similarly, Jin et al. [8] showed that complex and finely programmed biomimetic motions can be generated using a pneumatic actuator with a variable kirigami pattern (Figure 11d) [8]. Alternatively, as shown in Figure 11e, by varying a bilayer configuration along the length of a sample, for example by changing the passive layer position and thickness, it is possible to control the curvature locally [39]. Similarly, using an anisotropic passive layer in a bilayer configuration allows for the generation of bending in various directions or helixing [37,44]. With IPMC, Chang et al. [94] demonstrated that designing actuators with only one electrode side allows complex patterning and the generation of new kinematics [94].

Instead of varying the design, a simple module capable of simple kinematics can be repeated. These repeated modules can be stimulated together (simple modular assembly), as illustrated in Figure 11f, where all the modules are actuated by a unique pressure source [178], or in Figure 11g, where all active modules are actuated using the same heat source [80]. Alternatively, the different modules can be stimulated independently (complex modular assembly), as illustrated in Figure 11c [75] and Figure 11i [32]. Actuating the modules independently increases strongly the versatility of the actuator and the design and control complexity. For both strategies, various subclasses of modular assemblies 
can be defined. Table 3 redirects the reader to relevant works for each modular strategy. A widely studied type of module consists in tubular segments. Connolly et al. [178] studied the influence of fibre reinforcement on a segment kinematic and demonstrated the possibility to achieve complex motions with a single pressure source, such as finger biomimetism, by combining elongating and bending segments (Figure 11f) [178]. Alternatively, hinges mechanisms, consisting in small bending segments (hinges) in between larger rigid segments, allow for active origami-like shaping, usually with non-reconfigurable kinematics. As illustrated in Figure 11g with a simple modular assembly, [80] showed origami folding of rigid segments driven by thermally actuated LCE hinges [80]. Metamaterials can be seen as another class of modular assembly when made of active repeated cells. Passive auxetic structures and metamaterials have been widely studied and reviewed [191-193]. However, few works on active materials have been reported. Among them, Zhang et al. [59] proposed a swelling active metamateiral capable of isotropic and anisotropic contraction and tunable stress-strain curves, fixed by the unit cell design (Figure 11h) [59]. A promising type of modular assembly consists in the use of voxels. One voxel can be a given kinematics or reprogrammable. Each voxel is generally controlled by one or more stimulus, leading to complex leads management in the case of tethered transducers. Lin et al. [32] designed pneumatic voxels with active asymmetry that can be assembled in several ways and stimulated separately to achieve complex tasks (Figure 11i) [32]. What we call here rigid base modular assembly is illustrated in Figure $11 \mathrm{j}, \mathrm{k}[30,79]$. Several soft modules are assembled in a rigid structure, typically to form grippers or walkers. Other complex modular assemblies can be proposed. As an example, Sun et al. [92] developed a TCP-actuated arm composed of three independent modules [92].

\subsubsection{Advanced Strategies}

The complex designs and modular assemblies presented above, relying on several stimuli, present the advantages of leading to high versatility but the disadvantage of increasing the actuation complexity, since multiple stimuli are required. To overcome this limitation, several advanced strategies have been proposed. The use of the transducer nonlinearities has been studied to propose elegant strategies to achieve complex motions with limited stimulus complexity. Gorissen et al. [21] demonstrated sequential actuation of McKibben muscles with a single pressure source using the nonlinear pressure-volume curves of the muscles and the pressure drop of the leads. (Figure 111) [21]. Even if not implemented, Han et al. [16] proposed several strategies to obtain a stepped temperature response of a fluidic chamber filled with phase change fluid [16]. Pal et al. [10] used the release of elastic energy stored in a passive membrane when deformed pneumatically to program rapid deformation of soft structures [10].

Finally, using a complex controlled anisotropic stimulus proved efficient to achieve sequential motions or high versatility with a single stimulus, typically light or magnetic field. Palagi et al. [82] reported an LCE microactuator simulated by structured light (Figure 11m) [82]. This structured light actuates extremely locally and with a cyclic pattern on the LCE, generating swimming or other locomotion modes. Xu et al. [123] developed magnetic robots capable of complex tasks by using both design variation and a controlled anisotropic stimulus (Figure 11n) [123]. When placed in a variable and controlled magnetic field, the flexible robots will develop various motions depending on both their magnetization profile and the magnetic stimulation. These solutions present an advantage to allow fabrication of small-scale untethered robots.

\subsection{Complex Motions and Applications}

Using the presented strategies, a large class of complex motions can be achieved. Due to the large variety of motions achieved by soft robots, it is difficult to propose an exhaustive classification. Nam and Pei [194] proposed a complete taxonomy of 4D printing SMP behaviors that could be extended for other soft transducers and fabrication methods [194]. The complex motions are generally related to the task to achieve and hence to the target application. Using materials with stiffness similar to soft biological tissues, soft robotics has been developed as a bioinspired field [99,102,195]. 
Several applications have been identified pushing the development of complex soft robots: biomedical applications [4,104,196,197] (surgical tools [198,199], microrobots [200], rehabilitation and cell culture), human-machine interaction (haptic devices and wearables), locomotion, manipulation [5,201], microfluidic and structure self-assembly [202]. Towards those applications, the complex motions are classified here into several classes: soft arms, transportation, configuration changes and grippers. This is further detailed and illustrated in Table 4. Biomimetic motions can be identified transversely in this classification. This classification is not exhaustive but aims at providing an overview of the typical motions achieved in soft robotics and the corresponding target applications. Note that many papers provide impressive demonstrators but without targeting specific applications.

Table 4. Complex motions classification and their typical applications: black indicates a rigid material, and grey indicates the transducer.

\begin{tabular}{|c|c|c|c|}
\hline & Classification & Example & Typical Application \\
\hline \multirow{3}{*}{ Soft arm } & Simple bending & & \multirow{3}{*}{$\begin{array}{l}\text { Surgical device }[6,7,12,14,18,75,95] \\
\text { Manipulator }[10,15,22,28,44,90,92,159] \\
\text { Microfluidic }[40,44] \\
\text { Assistive device }[26] \\
\text { Agroforestery [203] } \\
\text { Not specified }[85,94,178]\end{array}$} \\
\hline & Multiple curvature & & \\
\hline & Helixing & & \\
\hline \multirow{4}{*}{ Transportation } & Walking/inching & & \multirow{4}{*}{$\begin{array}{l}\text { Locomotion }[11,13,17,21,71,80,82,84,179] \\
\text { Drug delivery }[72]\end{array}$} \\
\hline & Inchworm/crawling & & \\
\hline & Jumping/Rolling & & \\
\hline & Swimming & & \\
\hline \multirow{3}{*}{ Gripper } & Star gripper & & \multirow{3}{*}{$\begin{array}{l}\text { Manipulators }[28,37,74,139] \\
\text { Drug delivery }[47] \\
\text { Micromanipulation }[47,71,123]\end{array}$} \\
\hline & Finger gripper & & \\
\hline & Other gripper & & \\
\hline \multirow{6}{*}{$\begin{array}{l}\text { Configuration } \\
\text { change and } \\
\text { other motions }\end{array}$} & Shape mimicking/self-folding & & \multirow{6}{*}{$\begin{array}{l}\text { Optics }[44,81] \\
\text { Self-assembly-reconfigurable } \\
\text { structures }[8,41,43,48,57,65,69,69,80,88] \\
\text { Tunable material }[59,71] \\
\text { Surgical sutures }[66,69] \\
\text { Not specified }[89]\end{array}$} \\
\hline & Origami rigid self-folding & & \\
\hline & Self-tightening knot & (n) & \\
\hline & Active metamaterials & $\Delta$ & \\
\hline & Muscle like & & \\
\hline & Gaussian curvature & & \\
\hline
\end{tabular}

\subsubsection{Soft Arms}

A large variety of motions can be achieved by soft arm structures, from simple bending to complex trunk or octopus arm-like robots (Figure 11f,k), including simple helixing and multiple curvature systems (Figure 11c). This class gathers soft structure that precisely position an end effector relative to a fixed base. Such actuators often present a tubular shape. An important driving application for such actuators is 
the development of minimally invasive surgery devices. The STIFF-FLOP project strongly contributed to advances in this field [7,12], and the developed surgical module was validated on cadaver tests [204]. Similarly, Yeung et al. [205] reviewed soft pneumatic-based devices in colonoscopy that obtained Food and Drug Administration (FDA) approvals [205]. Alternatively, the development of versatile manipulators able to manipulate fragile objects has also be widely demonstrated, as illustrated in Figure 11k [79]. Other applications of such robots have been proposed, such as a soft arm for agroforestery [203].

\subsubsection{Transportation}

This class of motions gathers various types of mobile robots, often bioinspired. Among them, many robots capable of locomotion to reach space through tortuous paths or on unequal grounds have been demonstrated. In addition, the development of untethered microrobots for surgical procedures and drug delivery is the second driving application for this type of motions. Various strategies can be used to achieve transportation, as detailed in Table 4. Walking or inching robots are described here as robots standing on the ground via legs or segments and are able to produce a displacement, even by actuating them alternatively or by relying on an asymmetric actuation. An example is given in Figure 11n (untethered centipede robot). Similarly, inchworm or crawling robots lay directly on the ground and are generally tubular. They rely on sequential actuation of several segments or on an asymmetrical coefficient of friction with the ground. Jumping or rolling robots rely on quick shape configuration or on external dragging forces to move. They are often untethered. Finally, swimming robots are able to move in fluids thanks to oscillating motions. An example is shown in Figure $11 \mathrm{~m}$.

\subsubsection{Grippers}

Gripper-like motions have been widely studied and act as an important driving force for the development of soft robots. Shintake et al. [5] and Hughes et al. [201] reviewed soft gripper designs and capabilities $[5,201]$. The developed structures allow the manipulation of fragile or complex shape objects. Other potential applications usually mentioned are micromanipulation and drug delivery. In their classical configuration, bending soft segments are placed on a rigid structure. This class of soft robotics solution is one of the few to have reached the market today [5]. A common alternative design consists in what we call star gripping, consisting of monolithic grippers shaped as folding star, generally used on a smaller scale (Figure 11n). Additionally, various designs have been proposed in the literature.

\subsubsection{Configuration Change}

Finally, developing structures able to change their configuration and to morph into complex shapes is another ability enabled by soft stimuli-responsive transducers. This is strongly related to the concept of 4D printing, detailed in Section 4. Impressive demonstrators able to mimic biological and other complex shapes have been demonstrated, as illustrated in Figure 11d. Self folding structures, based typically on origami structures, allow to build complex and sometimes reconfigurable structures (Figure 11g). Rus and Tolley [206] reviewed active origami structures design, fabrication and control [206]. Muscle-like actuators, composed of rigid segments actuator by soft contractile muscles, is another typical bioinspired class of motion $[34,83]$. Complex shapes changes have also been demonstrated with active metamaterials structures [59,71], and self-tightening knots [66]. These solutions could find, among others, biomedical applications in deployable stents or for surgical sutures. Note that planar actuators able to develop Gaussian curvature are an important class but are not detailed in this review. For complete reviews on such actuators capabilities and principles, the authors redirect the reader to van Manen et al. [207]. As developed in Table 4, a variety of applications is proposed for such configuration change robots, but they generally remain at the stage of the demonstration. 


\section{Manufacturing}

Mechanical programming and generation of complex motions is indissociable from the manufacturing method. As stated in Section 2.4, asymmetry can be embedded in the transducer structure and the design possibilities are related to the chosen manufacturing method. Moreover, digital fabrication methods allow the introduction of anisotropy directly into the fabrication process and fine-tuning of the material properties locally, typically by creating gradients in the structures. Recently, several works focused more and more on integrated fabrication methods toward "walk out of the printer" robots. The future of the field is strongly related to $4 \mathrm{D}$ printing, where the fourth dimension corresponds to the material transformation over time when subjected to a given stimulus [104,189,196,208,209]. This section presents a general overview of manufacturing methods and how they enable mechanical programming. A quantitative comparison of the methods is proposed in Figure 12, highlighting the possibilities and limitation of each class of methods. Soft robotics is a multidisciplinary field, and fabrication methods gather works from material synthesis to design an oriented assembly of commercial components. Miriyev et al. [184] reviewed recent research in the field to understand the current challenges and focus of the soft robotics community and found an equivalent proportion between work describing the synthesis of materials and work based on existing materials [184]. Among the reviewed papers, 30\% of them presented the synthesis of a new material, while $60 \%$ of the works used off-the-shelf commercial materials and $10 \%$ modified commercial materials (material doping). Shaping the transducer material into an actuator, several classes of manufacturing methods are defined and compared here: 2D manufacturing, moulding, assembly and 3D printing methods.

\subsection{D Manufacturing}

2D manufacturing methods are defined here as processes allowing the fabrication of planar structures. Various methods have been identified in the reviewed works, as thermal compression [56], coating or layer deposition [95], photopatterning [46,48], laser cutting [8], and etching or photolithography [41]. They are generally relatively simple but limited in the achievable design complexities. Consequently, they are often used as a partial step of more complex fabrication methods, such as laser cutting. Among them, photopatterning methods are very efficient to control the material properties locally and to generate actuation gradients, typically by modulating transducer properties with the exposure time. Huang et al. [210] demonstrated this possibility with swelling materials [48] and $\mathrm{Xu}$ et al. [123] combined photopattenring with an imposed magnetic field to align magnetic particles and to polymerize selective part of structures, creating complex magnetization profiles [123].

\subsection{Moulding}

Moulding methods are also commonly used for soft robotics. In particular, this is an especially suitable method to shape elastomers used in many transducers. Here also, the general nomenclature of moulding gathers various processes. Moulding allows the fabrication of complex shapes, but the process complexity increases quickly when considering complex designs or submillimetric dimensions. It remains widely used, especially for a fluidic chamber actuator, but does not allow fine control of the transducer capabilities. 
(a)

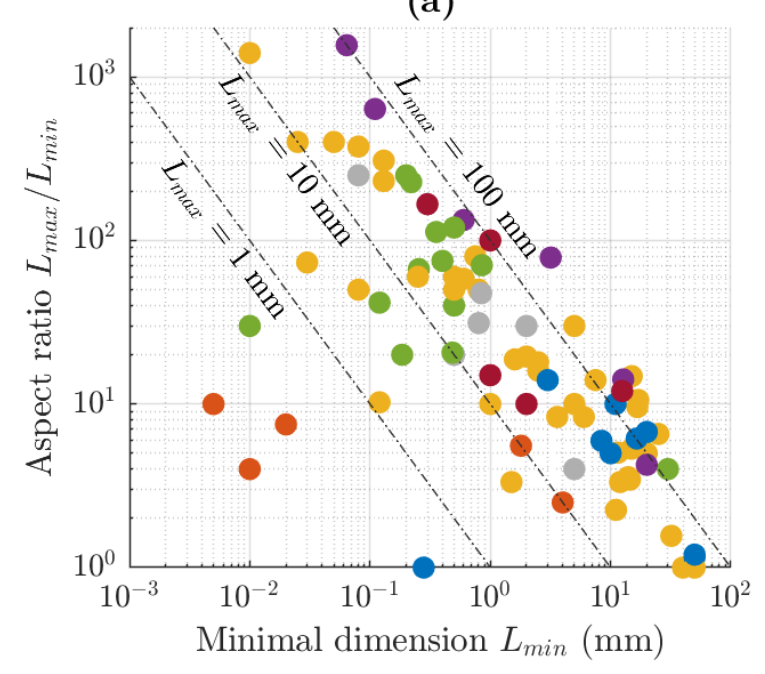

(b)

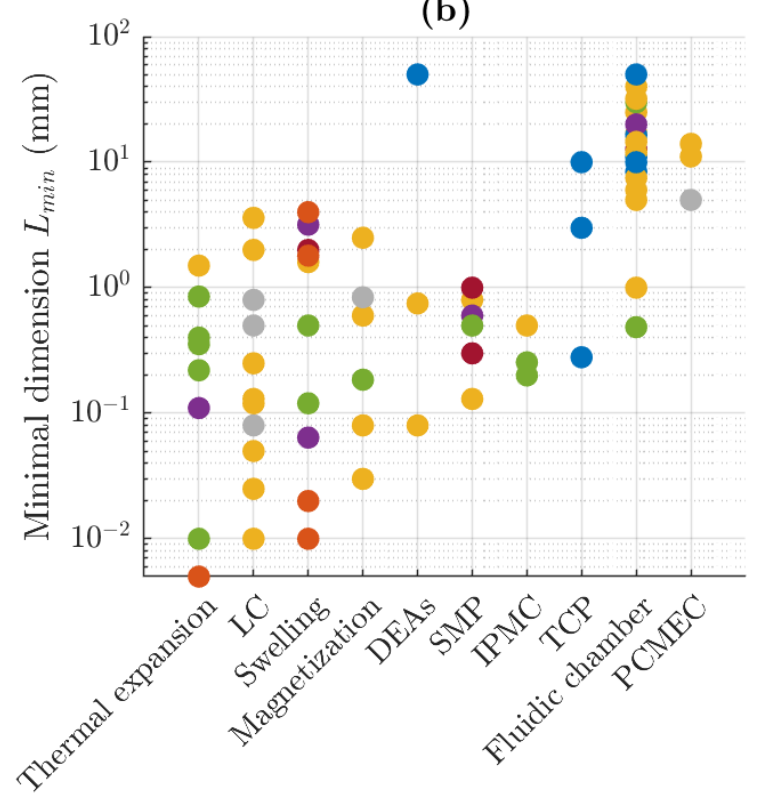

(c)

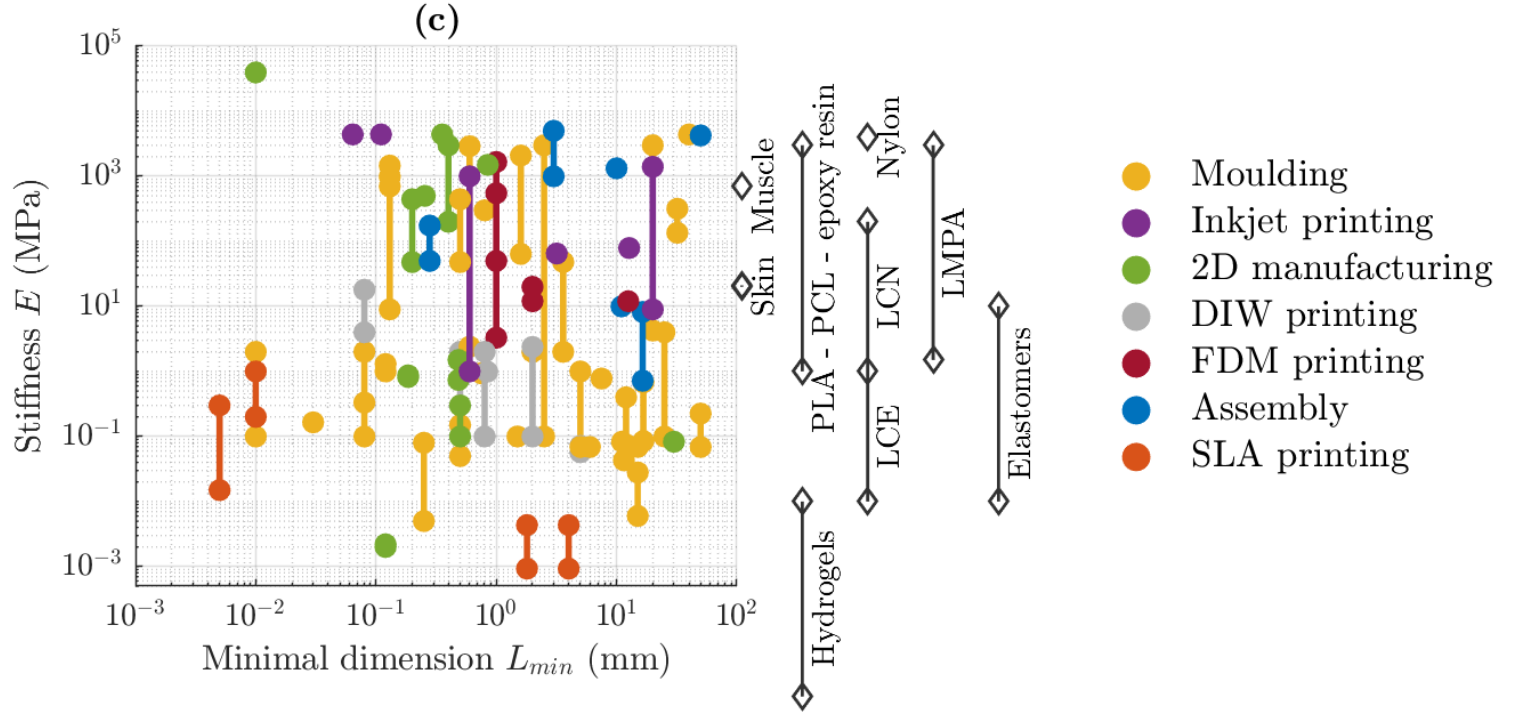

Figure 12. Comparison of different fabrication methods: each point or line corresponds to one actuator. The color of the marker corresponds to the class of the fabrication method. (a) Aspect ratio as a function of the minimal dimension. (b) Minimal dimension as a function of the transducer. (c) Stiffness as a function of the minimal dimension. See Figure A2 for references.

\subsection{Assembly}

Assembly methods are defined here as manufacturing techniques using directly-off-the-shelf components, with or without simple modifications, such as cutting, sewing, pasting and preloading. TCP actuators are typically made of assembled and preloaded nylon fibres. This method presents the advantage of being especially simple but is limited for downscaling reported designs.

\subsection{D Printing}

Three-dimensional printing methods for soft robotics have enable important progress in the field and have been thoroughly reviewed [211-213]. Several subclasses of 3D printing are usually defined: fused deposition modelling (FDM), where a thermoplastic filament is extruded to build the structure layer per layer; direct ink writing (DIW), using similar principles with a liquid ink that solidifies upon deposition; inkjet printing, where for each layer small droplets are deposited and then solidified 
selectively; stereolithography (SLA), where the object is built from a bath of photoresponsive material by selective pattern illumination of the layers; and selective laser sintering (SLS), where solid powder is melted selectively by a laser to be fused into the desired object. FDM, DIW, inkjet and SLA printing were used in the same proportion, while no reviewed work reported the use of SLS printing.

Even if limited in terms of small dimensions and adhesion between layers and adjacent filaments, access to FDM printing has been widely democratized in the last years, making it interesting to develop inexpensive and easily reproducible soft actuators. Furthermore, FDM technologies are the most promising candidates to develop structures made of multiple materials [189]. FDM is not limited to rigid polymers and has been demonstrated as a suitable fabrication process for pneumatic actuators [28]. The influence of printing parameters on the actuation characteristics of SMP has also been studied, allowing to fine-tune the transducer [67]. DIW printing is generally more limited to achieving complex shapes and a high number of layers because of the risk to see the printed structure collapsing before its solidification. Both FDM and DIW printing dimensions depend on the nozzle diameter [211]. Kim et al. [71] deposited aligned ferromagnetic particles in an ink filament to build complex structures with controlled magnetization profiles using a customized DIW setup [71]. Similarly, DIW has been demonstrated as efficient to align LCE chains in the deposited ink, allowing to create complex asymmetric shapes and patterns [80,81]. Inkjet printing, as SLA, allows higher resolution [211]. Ding et al. [65] preprogrammed SMP-elastomer composites using inkjet printing [65]. A compressive strain is introduced in the elastomer and controlled locally during photopolymerization by varying the layer's printing time. After printing and upon heating, the SMP softens and the constraint on the elastomer are released, allowing the structure to deform into a new permanent shape. Bodaghi et al. [69] applied the same principle with FDM printing [69]. Here, the SMP is pre-strained during extrusion depending on the nozzle speed. If reheated, for example during the deposition of a new layer, the pre-strain is reduced. It is then possible to control the pre-strain layer by layer. After printing, when heated, the constraints are released and the SMP takes a new permanent shape. Inkjet also allows high versatility for multimaterial fabrication. Zhang et al. [59] fabricated three-components swelling metamaterials [59], and Zhang et al. [25] used a similar process to fabricate a variable stiffness pneumatic actuator [25], both using commercial materials and printers. Finally, while SLA printing relies on photopolymerizable resins, it offers many possibilities in terms of designs, material development, scalability and speed of fabrication [189]. Odent et al. [49] demonstrated SLA possibilities to create cross-link densities and chemical composition gradients to create various bilayer configurations. Similarly, Hippler et al. [40] used 3D laser lithography to locally change the light exposure dose and to control the stimuli-responsiveness of hydrogel [40].

\subsection{Overview and Comparison}

When designing soft robots, the choice of a fabrication method will depend on several criteria, such as its dimensions, constitutive material, desired shape and need to create a gradient of stiffness or stimuli-responsiveness in the material. A quantitative comparison of stiffness, dimension and aspect ratio and the reviewed works based on the fabrication method is proposed in Figure 12. The references used can be found in Figure A2. Considering their sizes, two critical parameters are the minimal dimension of a given actuator and its aspect ratio. Reviewed works are mapped along those two axis in Figure 12a. The lines of a decreasing slope correspond to a constant maximal dimension. It can be highlighted from this representation that SLA methods allow for printing on a small scale but with a relatively low aspect ratio. Contrarily, assembly methods allow for easy manufacturing of large structures. It is also interesting to note that, if moulding allows a large range of minimal dimensions and aspect ratios, most of the actuators' maximal dimensions are on the centimeter scale. Two-dimensional manufacturing methods and inkjet printing emerge from this representation wtih high aspect ratios, covering a large range of minimal dimensions. Figure $12 b$ focuses on the minimal dimensions and fabrication methods used for the different transducers. Except for TCP (where the fibre needs to be twisted and coiled), no transducer depends on one single fabrication method. Finally, 
Figure 12c presents the actuators along their minimal dimension and their stiffness. Stiffnesses of typical constitutive materials are shown on the right side of the figure. Moulding and 2D manufacturing techniques, both gathering various classes of techniques, can be used to shape materials characterized by Young's modulus between less than $10 \mathrm{kPa}$ to more than $1 \mathrm{GPa}$. Focusing on 3D printing techniques, SLA can be used with softer materials, followed by DIW, FDM and inkjet printing. Additional to the criteria discussed above, the possibility to introduce anisotropy in the structure during the fabrication process is obviously an interesting feature, allowed by digital fabrication processes. As described above, it has been demonstrated with photopatterning to modify locally the material properties and with all the 3D printing techniques, even by changing locally the printing parameters or by embedding directionality by controlling the printing path.

\section{Perspectives}

Over the past years, soft stimuli-responsive materials demonstrated great potential for the design and fabrication of the next generation of actuators with controlled kinematics, thanks to remarkable architectures and compositions. Devices with increasing geometrical complexity and enhanced mechanical capabilities have been produced using innovative fabrication methods. Despite the latest progress in the field and the confirmed daily life application of these soft materials, some major challenges still remain unsolved.

(i) Conceptually, a close multidisciplinary collaboration between materials scientists and mechanical engineers plays a crucial role towards the fabrication of a new generation of responsive soft materials with advanced mechanical capabilities. Today, the chemical architecture of the macromolecules and the physicochemical properties conferred to the initial material are necessary but not sufficient to fabricate transducers having advanced mechanical capabilities. Further designs strategies must be used to integrate the desired anisotropy and to enable complex motions.

(ii) About the design, extremely various strategies have been proposed to introduce anisotropy and to develop complex motions. Those strategies are generally transversal, i.e., can be used with any transducer. However, some promising strategies remain less studied and lack methodological guidelines. In particular, controlled stiffness solutions present a great advantage for soft robotics. First, variable stiffness layers or patterns used as mechanical constraints can be used to finely adjust in real time the kinematics by controlling the asymmetry of the actuator. Secondly, it provides all the advantages of soft compliant structures while allowing locking of its shape and withstanding the applied forces in the stiff configuration. Furthermore, for reconfigurable and/or reprogrammable soft robots, modular or voxel based strategies seem particularly promising. Simple optimized voxels able to develop basic kinematics and made of any transducer can be printed or (re)assembled to generate complex and specific tasks.

(iii) Concerning the classification and comparison of the reviewed actuators, considerable efforts will still be needed to develop a complete framework allowing to describe, sort and compare the designs and capabilities of soft robots. Here, we have proposed an approach axed on the introduction of asymmetry. Several terms and concepts have been introduced or redefined to present and sort the actuators design ("intrinsic or extrinsic asymmetry", "passive and active asymmetry", etc.), their integration in soft robots ("complex motion generation strategy", "actuation multiplicity", etc.) and their capabilities ("basic kinematics", "complex motions", etc.). More, several terms remain ambiguously defined in terms of soft robotics (Does a continuous bending actuator have one or an infinite number of degrees of freedom?). Further works will be needed to propose a clear nomenclature to describe soft robots. Furthermore, when it comes to comparing them, the lack of adequate indicators can limit the discussion. The dimensions, actuation strain and stiffness are generally reported but can be ambiguously defined for soft active matter. Other criteria remain barely reported. In particular, the authors encourage the report of actuation stress, energy density, energy efficiency, number of cycles, weight, and density of new transducers and actuators. Finally, a better quantification of the anisotropy would be extremely beneficial for future soft robot designers, allowing to compare diverse ways to introduce it in the actuators designs. 
(iv) Considering the control of these emerging reconfigurable or continuous soft robots, new strategies as well as dedicated models and soft sensing strategies need to be established. Most of the reviewed work only presented open-loop control when not limited to on/off actuation. While in some cases the compliance limits the need for a controller, in most situations, the infinite number of degrees of freedom makes control even more difficult. The well-known challenges rely here on the modelling and sensor integration for continuous robots. Furthermore, even finer and innovative strategies will be required to control multiple stimuli-responsive, modular or reprogrammable actuators.

(v) About the technological aspects, the emerging additive manufacturing methods, such as 3D printing (SLA, SLS, FDM and bioprinting) are offering a still greater potential to fabricate active soft materials in one-step procedures. The proposed printing methods will enhance the fabrication of complex multi-components and multi-layered prototype devices with the targeted physicochemical properties and responsiveness. Currently, 3D printing focuses on the relatively easy printing of commercially available polymers, while the new materials face difficulties in terms of processability (scale-up, working temperature, filament production, restrictive access to the printing software for optimal working conditions, and others). Additional technological progress needs to be made to self-drive the fundamental research to its industrialization through 3D printing for personalized devices (e.g., biomimetic engineering application) with minimum fabrication cost. At this stage, new solutions are expected, as a result of a common effort based on scientific curiosity, imagination and creativity.

(vi) From the material science perspective, (nano)composites materials present great advantages for their various properties (electrical conductivity, light and magnetic sensitivity, and others) upon the incorporation of (nano)fillers such as CNT, plasmonic or magnetic particles. Moreover, the incorporation of such fillers also impact the materials' stiffness and stretchability, hence impacting the functional degrees of freedom during of the programmed motion. Exponential advances in the field are expected in polymer science, opening up new applications in soft robotics. Aside from the mechanical actuation capabilities of soft actuators, the perspective to integrate other functions, such as self-healing, biodegradability and sensing, is extremely appealing.

(vii) In line with the current environmental challenges and associated policy relative to the materials' recyclability and biodegradability, the question of the materials' life cycle will also remain a hot topic. In this context, material and engineer scientists will be even more challenged by going beyond the simple concept and degree of functionality of future soft-robotic devices. As a consequence, third parties will be involved for better elaboration and application of the procedures to be taken in order to generate human and environmental friendly devices.

\section{Conclusions}

With the present review, the authors proposed an ambitious approach to review the latest trends in soft robotics while focusing on programmable stimuli-responsive soft actuators. Strategies to generate complex motions from the kinematic capabilities of new materials, defined as transducers, have been studied. A multitude of solutions in the material selection and functionalization, the actuator design, and the fabrication possibilities have been detailed and compared quantitatively. Throughout this analysis and under the proposed design methodology framework, guidelines for materials scientists and engineers have been proposed. The domains of application and potential benefits of the presented actuators were highlighted and related to their kinematic capabilities and functionality. As a final remark, the authors outlined close future perspectives to be considered in the dynamic multidisciplinary cross-field between material science and robotics engineering.

Author Contributions: Conceptualization, G.D., A.T., L.B., T.L., J.-M.R., and P.L.; formal analysis, G.D.; investigation, G.D., A.T., and L.B.; writing—original draft preparation, G.D., A.T., and L.B.; writing-review and editing, P.L., J.-M.R., T.L., and A.D.; visualization, G.D.; supervision, P.L. All authors have read and agreed to the published version of the manuscript. 
Funding: This work was supported by the FNRS (Fonds National de la Recherche Scientifique) through two FRIA grants (G.D. and L.B., 2015-19) as well as the PREDICTION ARC project between Université Libre de Bruxelles and Université de Mons (2012-17) and FNRS PDR (no. 19471061, 2014-2018). This work was also supported by the Michel Cremer Foundation. A.T. thanks the BEWARE (no.410161) Fellowships Academia program cofunded by the COFUND program of the European Union (FP7-Marie Curie Actions) for the financial support. J.-M.R. as an F.R.S.-FNRS research associate and the LPCM thank the Belgian Federal Government Office of Science Policy (SSTC-PAI 6/27) for general support, and the LCFM-BIOMAT project is gratefully acknowledged.

Conflicts of Interest: The authors declare no conflict of interest. The funders had no role in the design of the study; in the collection, analyses, or interpretation of data; in the writing of the manuscript; or in the decision to publish the results.

\section{Abbreviations}

The following abbreviations are used in this manuscript:

$\begin{array}{ll}\text { Ag } & \text { silver } \\ \text { Au } & \text { gold } \\ \text { CNT } & \text { carbon nanotube } \\ \text { CTE } & \text { Coefficient of Thermal Expansion } \\ \text { DEA } & \text { Dielectric Elastomer Actuator } \\ \text { EAP } & \text { electroactive polymers } \\ \text { DIW } & \text { Direct Ink Writing } \\ \text { FDM } & \text { Fused Deposition Modelling } \\ \text { IPMC } & \text { Ionic Polymer Metal Composite } \\ \text { IR } & \text { Infrared } \\ \text { LC } & \text { Liquid Crystal } \\ \text { LCE } & \text { Liquid Crystal Elastomer } \\ \text { LCN } & \text { Liquid Crystal Network } \\ \text { LCP } & \text { Liquid Crystal Polymer } \\ \text { LMPA } & \text { Low Melting Point Alloy } \\ \text { MPCL } & \text { Methacrylated Polycaprolactone } \\ \text { MWCNT } & \text { Multi-Walled Carbon Nanotube } \\ \text { NPs } & \text { Nanoparticles } \\ \text { PCL } & \text { Polycaprolactone } \\ \text { PCMEC } & \text { Phase Change Material-Elastomer Composite } \\ \text { PDMS } & \text { Polydimethylsiloxane } \\ \text { PLA } & \text { Polylactide } \\ \text { PVA } & \text { poly(vinyl alcohol) } \\ \text { PUR } & \text { Polyurethane } \\ \text { SLA } & \text { Stereolithography } \\ \text { SLS } & \text { Selective Laser Sintering } \\ \text { SMA } & \text { Shape Memory Alloy } \\ \text { SMP } & \text { Shape Memory Polymer } \\ \text { TCP } & \text { Twisted and Coiled Polymer } \\ & \end{array}$




\section{Appendix A}

(a)

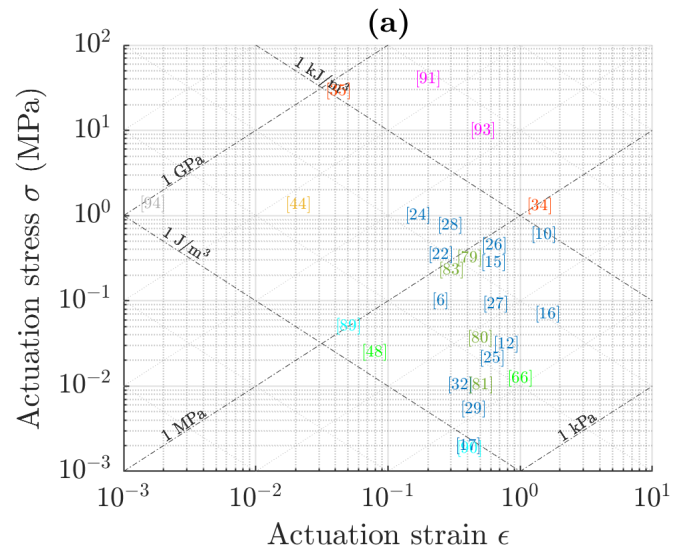

(c)

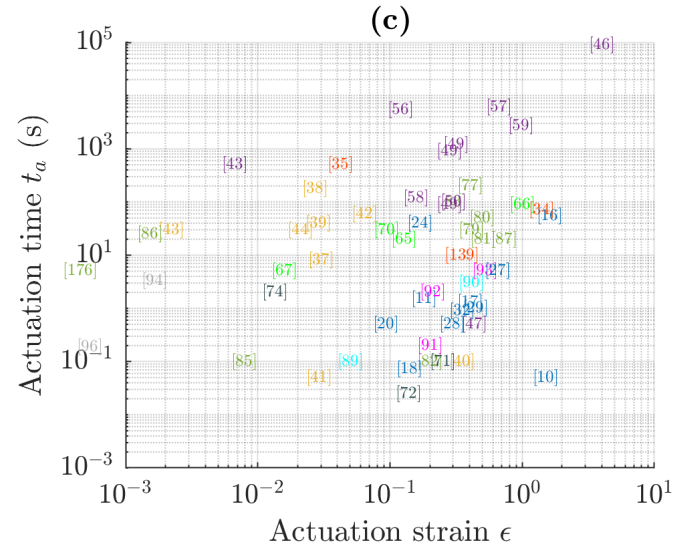

(e)

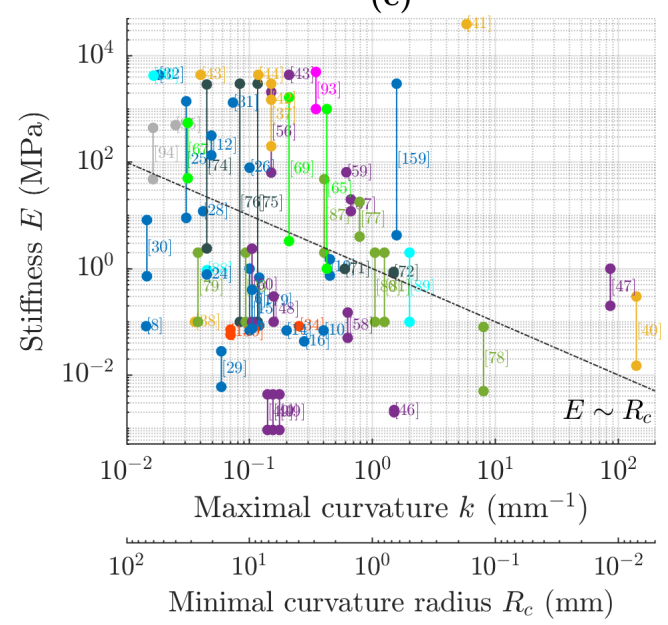

(b)

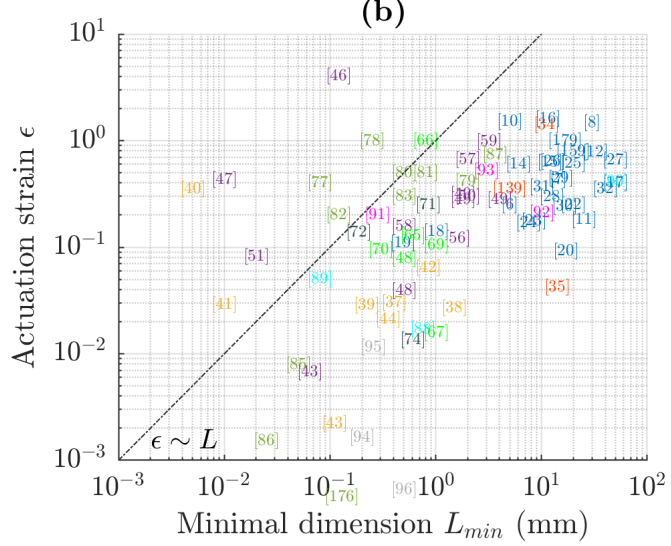

(d)

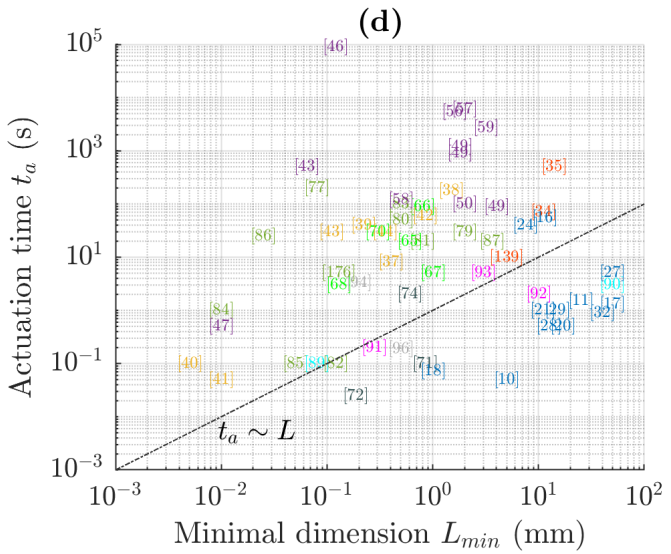

Figure A1. Comparison of the different actuators performances, with corresponding references. The colors represent the type of transducer. (a) Actuation stress as a function of the actuation strain. The diagonal lines of positive slope represent a constant value of $\sigma / \epsilon$, which is a characterization of the stiffness of the actuator. The diagonal lines of negative slope $\sigma \epsilon$ are lines of constant energy density. (b) Actuation strain as a function of the minimal dimension. The dashed line has a slope of $1(\epsilon \sim L)$. (c) Actuation time as a function of the actuation strain. (d) Actuation time as a function of the minimal dimension. The dashed line has a slope of $1\left(t_{a} \sim L\right)$. (e) Stiffness as a function of the curvature. The same figure without the references is presented and discussed in Figure 8 and a similar figure comparing the different extrinsic asymmetries is presented in Figure 9. 
(a)

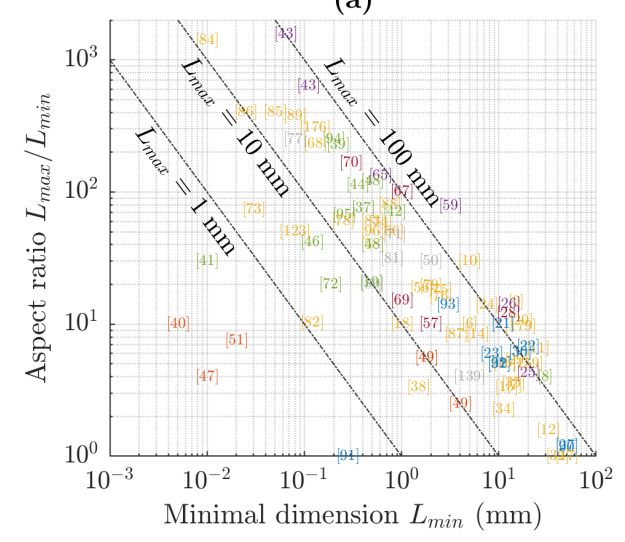

(c)

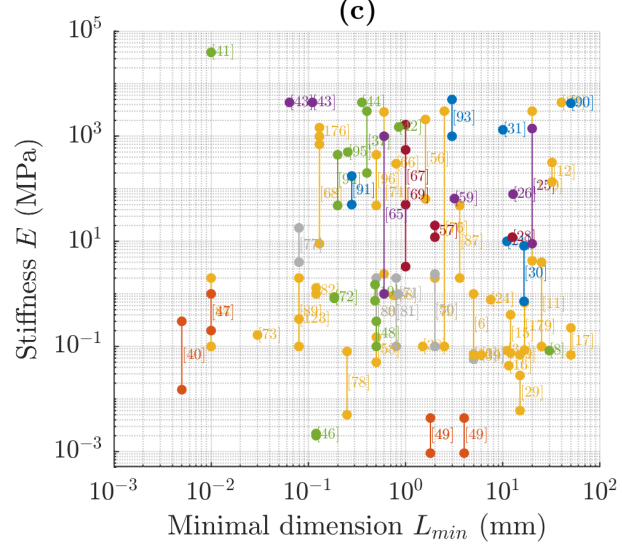

(b)

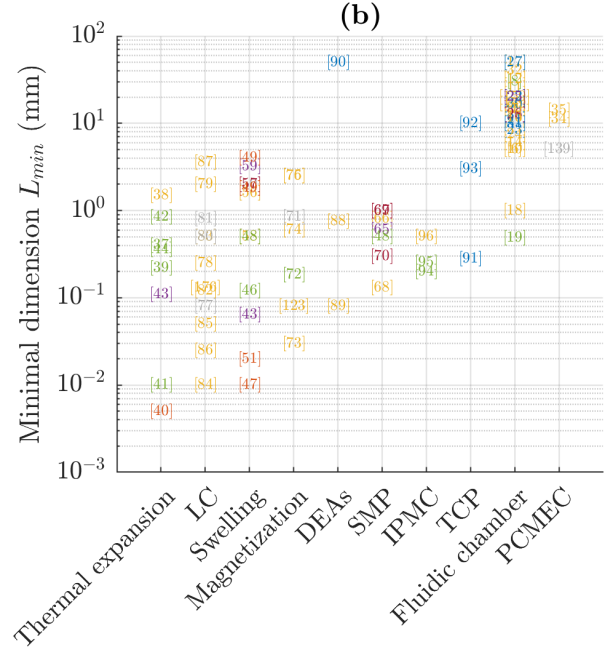

Moulding

- Inkjet printing

- 2D manufacturing

DIW printing

- FDM printing

- Assembly

- SLA printing

Figure A2. Comparison of the actuators based on the fabrication principles, with corresponding references: the colors represent the type of transducer. (a) Aspect ratio as a function of the minimal dimension. (b) Minimal dimension as a function of the transducer. (c) Stiffness as a function of the minimal dimension. The same figure without the references is presented and discussed in Figure 12.

Table A1. Comparison criteria for soft actuators to consider for the design and characterization of programmable soft robots, in addition to their kinematics capabilities: the solutions reviewed are compared based on these criteria. * Quantities used for the quantitative comparisons. ${ }^{\dagger}$ Normalized

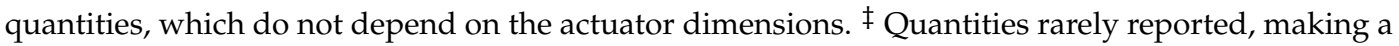
systematic comparison of the actuators difficult.

\begin{tabular}{|c|c|}
\hline Comparison Quantities & Units \\
\hline Generated linear force/blocking force & $\mathrm{N}$ \\
\hline Actuation stress $*,+$ & $\mathrm{MPa}$ \\
\hline Generated displacement/bending angle/curvature * & $\mathrm{mm} / \mathrm{rad} / \mathrm{mm}^{-1}$ \\
\hline Actuation strain $*,+$ & - \\
\hline Generated work/power $\ddagger$ & $\mathrm{J} / \mathrm{W}$ \\
\hline Energy density/specific energy density ${ }^{\dagger, \ddagger}$ & $\mathrm{kJ} \mathrm{m}^{-3} / \mathrm{J} \mathrm{kg}^{-1}$ \\
\hline Stimulus intensity & Depends on stimulus \\
\hline Energy conversion efficiency ${ }^{\dagger}$ & $\%$ \\
\hline Actuation time * & $\mathrm{s}$ \\
\hline Dimensions * & $\mathrm{mm}$ \\
\hline Aspect ratio $*,+$ & - \\
\hline Stiffness $*,+$ & $\mathrm{MPa}$ \\
\hline Number of cycles $\ddagger$ & - \\
\hline Weight & $\mathrm{kg}$ \\
\hline Density $^{\dagger}$ & $\mathrm{g} \mathrm{cm}^{-3}$ \\
\hline
\end{tabular}


Table A1. Cont.

\begin{tabular}{ll}
\hline Other Criteria & Description \\
\hline Reprogrammability & Ability to reprogram the kinematics \\
Possibility of remote actuation & Need of leads/connections (tethered/untethered) \\
Working environment & Typically air or water \\
Stimulus multiplicity & Possibility to actuate using different stimulus \\
Other capabilities & Sensing, self-healing, stiffness variation, etc. \\
Fabrication possibilities & See Section 4 \\
\hline
\end{tabular}

\section{References}

1. Rus, D.; Tolley, M.T. Design, Fabrication and Control of Soft Robots. Nature 2015, 521, 467-475. [CrossRef] [PubMed]

2. Majidi, C. Soft-Matter Engineering for Soft Robotics. Adv. Mater. Technol. 2019, 4, 1800477. [CrossRef]

3. Hines, L.; Petersen, K.; Lum, G.Z.; Sitti, M. Soft Actuators for Small-Scale Robotics. Adv. Mater. 2017, 29, 1603483. [CrossRef] [PubMed]

4. Cianchetti, M.; Laschi, C.; Menciassi, A.; Dario, P. Biomedical Applications of Soft Robotics. Nat. Rev. Mater. 2018, 3, 143-153. [CrossRef]

5. Shintake, J.; Cacucciolo, V.; Floreano, D.; Shea, H. Soft Robotic Grippers. Adv. Mater. 2018, 30, 1707035. [CrossRef]

6. Decroly, G.; Mertens, B.; Lambert, P.; Delchambre, A. Design, Characterization and Optimization of a Soft Fluidic Actuator for Minimally Invasive Surgery. Int. J. CARS 2020, 15, 333-340. [CrossRef]

7. Abidi, H.; Gerboni, G.; Brancadoro, M.; Fras, J.; Diodato, A.; Cianchetti, M.; Wurdemann, H.; Althoefer, K.; Menciassi, A. Highly Dexterous 2-Module Soft Robot for Intra-Organ Navigation in Minimally Invasive Surgery. Int. J. Med. Robot. Comput. Assist. Surg. 2018, 14, e1875. [CrossRef]

8. Jin, L.; Forte, A.E.; Deng, B.; Rafsanjani, A.; Bertoldi, K. Kirigami-Inspired Inflatables with Programmable Shapes. Adv. Mater. 2020, 32, 2001863. [CrossRef]

9. Milana, E.; Gorissen, B.; De Voider, M.; Reynaerts, D. Design of a Bi-Segmented Soft Actuator with Hardware Encoded Quasi-Static Inflation Sequence. In Proceedings of the 2018 IEEE International Conference on Soft Robotics (RoboSoft), Livorno, Italy, 24-28 April 2018; pp. 108-113. [CrossRef]

10. Pal, A.; Goswami, D.; Martinez, R.V. Elastic Energy Storage Enables Rapid and Programmable Actuation in Soft Machines. Adv. Funct. Mater. 2020, 30, 1906603. [CrossRef]

11. Rafsanjani, A.; Zhang, Y.; Liu, B.; Rubinstein, S.M.; Bertoldi, K. Kirigami Skins Make a Simple Soft Actuator Crawl. Sci. Robot. 2018, 3, eaar7555. [CrossRef]

12. Ranzani, T.; Gerboni, G.; Cianchetti, M.; Menciassi, A. A Bioinspired Soft Manipulator for Minimally Invasive Surgery. Bioinspir. Biomim. 2015, 10, 035008. [CrossRef] [PubMed]

13. Sui, X.; Cai, H.; Bie, D.; Zhang, Y.; Zhao, J.; Zhu, Y. Automatic Generation of Locomotion Patterns for Soft Modular Reconfigurable Robots. Appl. Sci. 2019, 10, 294. [CrossRef]

14. Sun, Y.; Song, S.; Liang, X.; Ren, H. A Miniature Soft Robotic Manipulator Based on Novel Fabrication Methods. IEEE Robot. Autom. Lett. 2016, 1, 617-623. [CrossRef]

15. Sun, Y.; Yap, H.K.; Liang, X.; Guo, J.; Qi, P.; Ang, M.H.; Yeow, C.H. Stiffness Customization and Patterning for Property Modulation of Silicone-Based Soft Pneumatic Actuators. Soft Robot. 2017, 4, 251-260. [CrossRef] [PubMed]

16. Han, J.; Jiang, W.; Niu, D.; Li, Y.; Zhang, Y.; Lei, B.; Liu, H.; Shi, Y.; Chen, B.; Yin, L.; et al. Untethered Soft Actuators by Liquid-Vapor Phase Transition: Remote and Programmable Actuation. Adv. Intell. Syst. 2019, 1, 1900109. [CrossRef]

17. Zou, J.; Lin, Y.; Ji, C.; Yang, H. A Reconfigurable Omnidirectional Soft Robot Based on Caterpillar Locomotion. Soft Robot. 2018, 5, 164-174. [CrossRef]

18. Gorissen, B.; De Volder, M.; Reynaerts, D. Chip-on-Tip Endoscope Incorporating a Soft Robotic Pneumatic Bending Microactuator. Biomed. Microdev. 2018, 20, 73. [CrossRef]

19. Sinatra, N.R.; Ranzani, T.; Vlassak, J.J.; Parker, K.K.; Wood, R.J. Nanofiber-Reinforced Soft Fluidic Micro-Actuators. J. Micromech. Microeng. 2018, 28, 084002. [CrossRef] 
20. Furukawa, S.; Wakimoto, S.; Kanda, T.; Hagihara, H. A Soft Master-Slave Robot Mimicking Octopus Arm Structure Using Thin Artificial Muscles and Wire Encoders. Actuators 2019, 8, 40. [CrossRef]

21. Gorissen, B.; Milana, E.; Baeyens, A.; Broeders, E.; Christiaens, J.; Collin, K.; Reynaerts, D.; Volder, M. Hardware Sequencing of Inflatable Nonlinear Actuators for Autonomous Soft Robots. Adv. Mater. 2019, 31, 1804598. [CrossRef]

22. Guan, Q.; Sun, J.; Liu, Y.; Wereley, N.M.; Leng, J. Novel Bending and Helical Extensile/Contractile Pneumatic Artificial Muscles Inspired by Elephant Trunk. Soft Robot. 2020. [CrossRef] [PubMed]

23. Kawamura, S.; Sudani, M.; Deng, M.; Noge, Y.; Wakimoto, S. Modeling and System Integration for a Thin Pneumatic Rubber 3-DOF Actuator. Actuators 2019, 8, 32. [CrossRef]

24. Matsuoka, H.; Kanda, T.; Wakimoto, S.; Suzumori, K.; Lambert, P. Development of a Rubber Soft Actuator Driven with Gas/Liquid Phase Change. Int. J. Autom. Technol. 2016, 10, 517-524. [CrossRef]

25. Zhang, Y.F.; Zhang, N.; Hingorani, H.; Ding, N.; Wang, D.; Yuan, C.; Zhang, B.; Gu, G.; Ge, Q. Fast-Response, Stiffness-Tunable Soft Actuator by Hybrid Multimaterial 3D Printing. Adv. Funct. Mater. 2019, 29, 1806698. [CrossRef]

26. Cappello, L.; Galloway, K.C.; Sanan, S.; Wagner, D.A.; Granberry, R.; Engelhardt, S.; Haufe, F.L.; Peisner, J.D.; Walsh, C.J. Exploiting Textile Mechanical Anisotropy for Fabric-Based Pneumatic Actuators. Soft Robot. 2018, 5, 662-674. [CrossRef]

27. Yang, H.D.; Greczek, B.T.; Asbeck, A.T. Modeling and Analysis of a High-Displacement Pneumatic Artificial Muscle With Integrated Sensing. Front. Robot. AI 2019, 5, 136. [CrossRef]

28. Hu, W.; Alici, G. Bioinspired Three-Dimensional-Printed Helical Soft Pneumatic Actuators and Their Characterization. Soft Robot. 2020, 7, 267-282. [CrossRef]

29. Yuk, H.; Lin, S.; Ma, C.; Takaffoli, M.; Fang, N.X.; Zhao, X. Hydraulic Hydrogel Actuators and Robots Optically and Sonically Camouflaged in Water. Nat. Commun. 2017, 8, 14230. [CrossRef]

30. Terryn, S.; Roels, E.; Brancart, J.; Van Assche, G.; Vanderborght, B. Self-Healing and High Interfacial Strength in Multi-Material Soft Pneumatic Robots via Reversible Diels-Alder Bonds. Actuators 2020, 9, 34. [CrossRef]

31. Liao, B.; Zang, H.; Liu, Y.; Wang, Y.; Lang, X.; Jin, J.; Zhu, N.; Yin, Q. Programmable Design of Soft Actuators and Robots. In Proceedings of the 2019 WRC Symposium on Advanced Robotics and Automation (WRC SARA), Beijing, China, 21-22 August 2019; pp. 222-227. [CrossRef]

32. Lin, Y.; Yang, G.; Liang, Y.; Zhang, C.; Wang, W.; Qian, D.; Yang, H.; Zou, J. Controllable Stiffness Origami "Skeletons" for Lightweight and Multifunctional Artificial Muscles. Adv. Funct. Mater. 2020, 30, 2000349. [CrossRef]

33. Baumgartner, M.; Hartmann, F.; Drack, M.; Preninger, D.; Wirthl, D.; Gerstmayr, R.; Lehner, L.; Mao, G.; Pruckner, R.; Demchyshyn, S.; et al. Resilient yet Entirely Degradable Gelatin-Based Biogels for Soft Robots and Electronics. Nat. Mater. 2020, 19, 1102-1109. [CrossRef] [PubMed]

34. Miriyev, A.; Stack, K.; Lipson, H. Soft Material for Soft Actuators. Nat. Commun. 2017, 8, 596. [CrossRef] [PubMed]

35. Lipton, J.I.; Angle, S.; Banai, R.E.; Peretz, E.; Lipson, H. Electrically Actuated Hydraulic Solids: Electrically Actuated Hydraulic Solids. Adv. Eng. Mater. 2016, 18, 1710-1715. [CrossRef]

36. Li, L.; Scheiger, J.M.; Levkin, P.A. Design and Applications of Photoresponsive Hydrogels. Adv. Mater. 2019, 31, 1807333. [CrossRef] [PubMed]

37. Li, Q.; Liu, C.; Fan, S. Programmable and Functional Electrothermal Bimorph Actuators Based on Large-Area Anisotropic Carbon Nanotube Paper. Nanotechnology 2018, 29, 175503. [CrossRef]

38. Taniguchi, T.; Blanc, L.; Asahi, T.; Koshima, H.; Lambert, P. Statistical Modeling of Photo-Bending Actuation of Hybrid Silicones Mixed with Azobenzene Powder. Actuators 2019, 8, 68. [CrossRef]

39. Yao, S.; Cui, J.; Cui, Z.; Zhu, Y. Soft Electrothermal Actuators Using Silver Nanowire Heaters. Nanoscale 2017, 9, 3797-3805. [CrossRef]

40. Hippler, M.; Blasco, E.; Qu, J.; Tanaka, M.; Barner-Kowollik, C.; Wegener, M.; Bastmeyer, M. Controlling the Shape of 3D Microstructures by Temperature and Light. Nat. Commun. 2019, 10, 232. [CrossRef]

41. Zhu, Y.; Birla, M.; Oldham, K.R.; Filipov, E.T. Elastically and Plastically Foldable Electrothermal Micro-Origami for Controllable and Rapid Shape Morphing. Adv. Funct. Mater. 2020, 2003741. [CrossRef]

42. Sachyani Keneth, E.; Scalet, G.; Layani, M.; Tibi, G.; Degani, A.; Auricchio, F.; Magdassi, S. Pre-Programmed Tri-Layer Electro-Thermal Actuators Composed of Shape Memory Polymer and Carbon Nanotubes. Soft Robot. 2020, 7, 123-129. [CrossRef] 
43. Shigemune, H.; Maeda, S.; Hara, Y.; Hosoya, N.; Hashimoto, S. Origami Robot: A Self-Folding Paper Robot With an Electrothermal Actuator Created by Printing. IEEE/ASME Trans. Mechatron. 2016, 21, 2746-2754. [CrossRef]

44. Hu, Y.; Xu, A.; Liu, J.; Yang, L.; Chang, L.; Huang, M.; Gu, W.; Wu, G.; Lu, P.; Chen, W.; et al. Multifunctional Soft Actuators Based on Anisotropic Paper/Polymer Bilayer Toward Bioinspired Applications. Adv. Mater. Technol. 2019, 4, 1800674. [CrossRef]

45. Gunes, I.S.; Cao, F.; Jana, S.C. Evaluation of Nanoparticulate Fillers for Development of Shape Memory Polyurethane Nanocomposites. Polymer 2008, 49, 2223-2234. [CrossRef]

46. Cangialosi, A.; Yoon, C.; Liu, J.; Huang, Q.; Guo, J.; Nguyen, T.D.; Gracias, D.H.; Schulman, R. DNA Sequence-Directed Shape Change of Photopatterned Hydrogels via High-Degree Swelling. Science 2017, 357, 1126-1130. [CrossRef] [PubMed]

47. Hu, Y.; Wang, Z.; Jin, D.; Zhang, C.; Sun, R.; Li, Z.; Hu, K.; Ni, J.; Cai, Z.; Pan, D.; et al. Botanical-Inspired 4D Printing of Hydrogel at the Microscale. Adv. Funct. Mater. 2020, 30, 1907377. [CrossRef]

48. Huang, L.; Jiang, R.; Wu, J.; Song, J.; Bai, H.; Li, B.; Zhao, Q.; Xie, T. Ultrafast Digital Printing toward 4D Shape Changing Materials. Adv. Mater. 2017, 29, 1605390. [CrossRef]

49. Odent, J.; Vanderstappen, S.; Toncheva, A.; Pichon, E.; Wallin, T.J.; Wang, K.; Shepherd, R.F.; Dubois, P.; Raquez, J.M. Hierarchical Chemomechanical Encoding of Multi-Responsive Hydrogel Actuators Via 3D Printing. J. Mater. Chem. A 2019, 7, 15395-15403. [CrossRef]

50. Zheng, S.Y.; Shen, Y.; Zhu, F.; Yin, J.; Qian, J.; Fu, J.; Wu, Z.L.; Zheng, Q. Programmed Deformations of 3D-Printed Tough Physical Hydrogels with High Response Speed and Large Output Force. Adv. Funct. Mater. 2018, 28, 1803366. [CrossRef]

51. Guo, Y.; Shahsavan, H.; Sitti, M. 3D Microstructures of Liquid Crystal Networks with Programmed Voxelated Director Fields. Adv. Mater. 2020, 2002753. [CrossRef]

52. Kalita, H.; Mandal, M.; Karak, N. Biodegradable Solvent-Induced Shape-Memory Hyperbranched Polyurethane. J. Polym. Res. 2012, 19, 9982. [CrossRef]

53. Lu, H.; Liu, Y.; Leng, J.; Du, S. Qualitative Separation of the Effect of the Solubility Parameter on the Recovery Behavior of Shape-Memory Polymer. Smart Mater. Struct. 2009, 18, 085003. [CrossRef]

54. Yu, K.; Zhang, Z.; Liu, Y.; Leng, J. Carbon Nanotube Chains in a Shape Memory Polymer/Carbon Black Composite: To Significantly Reduce the Electrical Resistivity. Appl. Phys. Lett. 2011, 98, 074102. [CrossRef]

55. Bai, Y.; Chen, Y.; Wang, Q.; Wang, T. Poly(Vinyl Butyral) Based Polymer Networks with Dual-Responsive Shape Memory and Self-Healing Properties. J. Mater. Chem. A 2014, 2, 9169. [CrossRef]

56. Toncheva, A.; Willocq, B.; Khelifa, F.; Douheret, O.; Lambert, P.; Dubois, P.; Raquez, J.M. Bilayer Solvent and Vapor-Triggered Actuators Made of Cross-Linked Polymer Architectures via Diels-Alder Pathways. J. Mater. Chem. B 2017, 5, 5556-5563. [CrossRef]

57. Baker, A.B.; Bates, S.R.; Llewellyn-Jones, T.M.; Valori, L.P.; Dicker, M.P.; Trask, R.S. 4D Printing with Robust Thermoplastic Polyurethane Hydrogel-Elastomer Trilayers. Mater. Des. 2019, 163, 107544. [CrossRef]

58. Alexander, S.L.M.; Ahmadmehrabi, S.; Korley, L.T.J. Programming Shape and Tailoring Transport: Advancing Hygromorphic Bilayers with Aligned Nanofibers. Soft Matter 2017, 13, 5589-5596. [CrossRef]

59. Zhang, H.; Guo, X.; Wu, J.; Fang, D.; Zhang, Y. Soft Mechanical Metamaterials with Unusual Swelling Behavior and Tunable Stress-Strain Curves. Sci. Adv. 2018, 4, eaar8535. [CrossRef]

60. Du, H.; Zhang, J. Solvent Induced Shape Recovery of Shape Memory Polymer Based on Chemically Cross-Linked Poly(Vinyl Alcohol). Soft Matter 2010, 6, 3370. [CrossRef]

61. Qi, X.; Yao, X.; Deng, S.; Zhou, T.; Fu, Q. Water-Induced Shape Memory Effect of Graphene Oxide Reinforced Polyvinyl Alcohol Nanocomposites. J. Mater. Chem. A 2014, 2, 2240-2249. [CrossRef]

62. Zhu, Y.; Hu, J.; Luo, H.; Young, R.J.; Deng, L.; Zhang, S.; Fan, Y.; Ye, G. Rapidly Switchable Water-Sensitive Shape-Memory Cellulose/Elastomer Nano-Composites. Soft Matter 2012, 8, 2509. [CrossRef]

63. Quitmann, D.; Gushterov, N.; Sadowski, G.; Katzenberg, F.; Tiller, J.C. Solvent-Sensitive Reversible Stress-Response of Shape Memory Natural Rubber. ACS Appl. Mater. Interfaces 2013, 5, 3504-3507. [CrossRef] [PubMed]

64. Zhao, Q.; Dunlop, J.W.C.; Qiu, X.; Huang, F.; Zhang, Z.; Heyda, J.; Dzubiella, J.; Antonietti, M.; Yuan, J. An Instant Multi-Responsive Porous Polymer Actuator Driven by Solvent Molecule Sorption. Nat. Commun. 2014, 5, 4293. [CrossRef] [PubMed] 
65. Ding, Z.; Yuan, C.; Peng, X.; Wang, T.; Qi, H.J.; Dunn, M.L. Direct 4D Printing via Active Composite Materials. Sci. Adv. 2017, 3, e1602890. [CrossRef] [PubMed]

66. Toncheva, A.; Khelifa, F.; Paint, Y.; Voué, M.; Lambert, P.; Dubois, P.; Raquez, J.M. Fast IR-Actuated Shape-Memory Polymers Using in Situ Silver Nanoparticle-Grafted Cellulose Nanocrystals. ACS Appl. Mater. Interfaces 2018, 10, 29933-29942. [CrossRef] [PubMed]

67. Wang, J.; Wang, Z.; Song, Z.; Ren, L.; Liu, Q.; Ren, L. Biomimetic Shape-Color Double-Responsive 4D Printing. Adv. Mater. Technol. 2019, 4, 1900293. [CrossRef]

68. Liu, J.A.C.; Gillen, J.H.; Mishra, S.R.; Evans, B.A.; Tracy, J.B. Photothermally and Magnetically Controlled Reconfiguration of Polymer Composites for Soft Robotics. Sci. Adv. 2019, 5, eaaw2897. [CrossRef]

69. Bodaghi, M.; Damanpack, A.; Liao, W. Adaptive Metamaterials by Functionally Graded 4D Printing. Mater. Des. 2017, 135, 26-36. [CrossRef]

70. Zolfagharian, A.; Kaynak, A.; Khoo, S.Y.; Kouzani, A. Pattern-Driven 4D Printing. Sens. Actuators A Phys. 2018, 274, 231-243. [CrossRef]

71. Kim, Y.; Yuk, H.; Zhao, R.; Chester, S.A.; Zhao, X. Printing Ferromagnetic Domains for Untethered Fast-Transforming Soft Materials. Nature 2018, 558, 274-279. [CrossRef]

72. Hu, W.; Lum, G.Z.; Mastrangeli, M.; Sitti, M. Small-Scale Soft-Bodied Robot with Multimodal Locomotion. Nature 2018, 554, 81-85. [CrossRef]

73. Zhang, J.; Diller, E. Untethered Miniature Soft Robots: Modeling and Design of a Millimeter-Scale Swimming Magnetic Sheet. Soft Robot. 2018, 5, 761-776. [CrossRef] [PubMed]

74. Ze, Q.; Kuang, X.; Wu, S.; Wong, J.; Montgomery, S.M.; Zhang, R.; Kovitz, J.M.; Yang, F.; Qi, H.J.; Zhao, R. Magnetic Shape Memory Polymers with Integrated Multifunctional Shape Manipulation. Adv. Mater. 2020, 32, 1906657. [CrossRef]

75. Chautems, C.; Tonazzini, A.; Boehler, Q.; Jeong, S.H.; Floreano, D.; Nelson, B.J. Magnetic Continuum Device with Variable Stiffness for Minimally Invasive Surgery. Adv. Intell. Syst. 2020, 2, 1900086. [CrossRef]

76. Chautems, C.; Tonazzini, A.; Floreano, D.; Nelson, B.J. A Variable Stiffness Catheter Controlled with an External Magnetic Field. In Proceedings of the 2017 IEEE/RSJ International Conference on Intelligent Robots and Systems (IROS), Vancouver, BC, Canada, 24-28 September 2017; pp. 181-186. [CrossRef]

77. Ambulo, C.P.; Burroughs, J.J.; Boothby, J.M.; Kim, H.; Shankar, M.R.; Ware, T.H. Four-Dimensional Printing of Liquid Crystal Elastomers. ACS Appl. Mater. Interfaces 2017, 9, 37332-37339. [CrossRef] [PubMed]

78. Barnes, M.; Verduzco, R. Direct Shape Programming of Liquid Crystal Elastomers. Soft Matter 2019, 15, 870-879. [CrossRef] [PubMed]

79. He, Q.; Wang, Z.; Wang, Y.; Minori, A.; Tolley, M.T.; Cai, S. Electrically Controlled Liquid Crystal Elastomer-Based Soft Tubular Actuator with Multimodal Actuation. Sci. Adv. 2019, 5, eaax5746. [CrossRef] [PubMed]

80. Kotikian, A.; McMahan, C.; Davidson, E.C.; Muhammad, J.M.; Weeks, R.D.; Daraio, C.; Lewis, J.A. Untethered Soft Robotic Matter with Passive Control of Shape Morphing and Propulsion. Sci. Robot. 2019, 4, eaax7044. [CrossRef] [PubMed]

81. López-Valdeolivas, M.; Liu, D.; Broer, D.J.; Sánchez-Somolinos, C. 4D Printed Actuators with Soft-Robotic Functions. Macromol. Rapid Commun. 2018, 39, 1700710. [CrossRef]

82. Palagi, S.; Mark, A.G.; Reigh, S.Y.; Melde, K.; Qiu, T.; Zeng, H.; Parmeggiani, C.; Martella, D.; Sanchez-Castillo, A.; Kapernaum, N.; et al. Structured Light Enables Biomimetic Swimming and Versatile Locomotion of Photoresponsive Soft Microrobots. Nat. Mater 2016, 15, 647-653. [CrossRef]

83. Wang, Y.; Wang, Z.; He, Q.; Iyer, P.; Cai, S. Electrically Controlled Soft Actuators with Multiple and Reprogrammable Actuation Modes. Adv. Intell. Syst. 2020, 2, 1900177. [CrossRef]

84. Zeng, H.; Wani, O.M.; Wasylczyk, P.; Priimagi, A. Light-Driven, Caterpillar-Inspired Miniature Inching Robot. Macromol. Rapid Commun. 2018, 39, 1700224. [CrossRef] [PubMed]

85. Lahikainen, M.; Zeng, H.; Priimagi, A. Design Principles for Non-Reciprocal Photomechanical Actuation. Soft Matter 2020, 16, 5951-5958. [CrossRef] [PubMed]

86. Ryabchun, A.; Li, Q.; Lancia, F.; Aprahamian, I.; Katsonis, N. Shape-Persistent Actuators from Hydrazone Photoswitches. J. Am. Chem. Soc. 2019, 141, 1196-1200. [CrossRef] [PubMed]

87. Yu, L.; Peng, R.; Rivers, G.; Zhang, C.; Si, P.; Zhao, B. Multifunctional Liquid Crystal Polymer Network Soft Actuators. J. Mater. Chem. A 2020, 8, 3390-3396. [CrossRef] 
88. Hajiesmaili, E.; Clarke, D.R. Reconfigurable Shape-Morphing Dielectric Elastomers Using Spatially Varying Electric Fields. Nat. Commun. 2019, 10, 183. [CrossRef]

89. Davidson, Z.S.; Shahsavan, H.; Aghakhani, A.; Guo, Y.; Hines, L.; Xia, Y.; Yang, S.; Sitti, M. Monolithic Shape-Programmable Dielectric Liquid Crystal Elastomer Actuators. Sci. Adv. 2019, 5, eaay0855. [CrossRef] [PubMed]

90. Xing, Z.; Zhang, J.; McCoul, D.; Cui, Y.; Sun, L.; Zhao, J. A Super-Lightweight and Soft Manipulator Driven by Dielectric Elastomers. Soft Robot. 2020, 7, 512-520. [CrossRef]

91. Haines, C.S.; Lima, M.D.; Li, N.; Spinks, G.M.; Foroughi, J.; Madden, J.D.W.; Kim, S.H.; Fang, S.; Jung de Andrade, M.; Goktepe, F.; et al. Artificial Muscles from Fishing Line and Sewing Thread. Science 2014, 343, 868-872. [CrossRef]

92. Sun, J.; Tighe, B.; Liu, Y.; Zhao, J. Twisted-and-Coiled Actuators with Free Strokes Enable Soft Robots with Programmable Motions. Soft Robot. 2020. [CrossRef]

93. Wu, C.; Zheng, W. A Modeling of Twisted and Coiled Polymer Artificial Muscles Based on Elastic Rod Theory. Actuators 2020, 9, 25. [CrossRef]

94. Chang, L.; Yu, L.; Li, C.; Niu, Q.; Hu, Y.; Lu, P.; Zhu, Z.; Wu, Y. Ionic Polymer with Single-Layered Electrodes: A Novel Strategy for Ionic Actuator Design. Smart Mater. Struct. 2018, 27, 105046. [CrossRef]

95. Tripathi, A.S.; Chattopadhyay, B.P.; Das, S. Cost-Effective Fabrication of Ionic Polymer Based Artificial Muscles for Catheter-Guidewire Maneuvering Application. Microsyst. Technol. 2019, 25, 1129-1136. [CrossRef]

96. Zhu, Z.; Bian, C.; Ru, J.; Bai, W.; Chen, H. Rapid Deformation of IPMC under a High Electrical Pulse Stimulus Inspired by Action Potential. Smart Mater. Struct. 2018, 28, 01LT01. [CrossRef]

97. Hao, M.; Wang, Y.; Zhu, Z.; He, Q.; Zhu, D.; Luo, M. A Compact Review of IPMC as Soft Actuator and Sensor: Current Trends, Challenges, and Potential Solutions From Our Recent Work. Front. Robot. AI 2019, 6, 129. [CrossRef]

98. Yang, Y.; Wu, Y.; Li, C.; Yang, X.; Chen, W. Flexible Actuators for Soft Robotics. Adv. Intell. Syst. 2020, 2, 1900077. [CrossRef]

99. Coyle, S.; Majidi, C.; LeDuc, P.; Hsia, K.J. Bio-Inspired Soft Robotics: Material Selection, Actuation, and Design. Extrem. Mech. Lett. 2018, 22, 51-59. [CrossRef]

100. McCracken, J.M.; Donovan, B.R.; White, T.J. Materials as Machines. Adv. Mater. 2020, 32, 1906564. [CrossRef] [PubMed]

101. Boyraz, P.; Runge, G.; Raatz, A. An Overview of Novel Actuators for Soft Robotics. Actuators 2018, 7, 48. [CrossRef]

102. Palagi, S.; Fischer, P. Bioinspired Microrobots. Nat. Rev. Mater. 2018, 3, 113-124. [CrossRef]

103. Rich, S.I.; Wood, R.J.; Majidi, C. Untethered Soft Robotics. Nat. Electron. 2018, 1, 102-112. [CrossRef]

104. Lui, Y.S.; Sow, W.T.; Tan, L.P.; Wu, Y.; Lai, Y.; Li, H. 4D Printing and Stimuli-Responsive Materials in Biomedical Aspects. Acta Biomater. 2019, 92, 19-36. [CrossRef] [PubMed]

105. Laschi, C.; Mazzolai, B.; Cianchetti, M. Soft Robotics: Technologies and Systems Pushing the Boundaries of Robot Abilities. Sci. Robot. 2016, 1, eaah3690. [CrossRef] [PubMed]

106. McEvoy, M.A.; Correll, N. Materials That Couple Sensing, Actuation, Computation, and Communication. Science 2015, 347. [CrossRef] [PubMed]

107. Koshima, H. (Ed.) Mechanically Responsive Materials for Soft Robotics, 1st ed.; Wiley: Hoboken, NJ, USA, 2020. [CrossRef]

108. Sun, L.; Huang, W.M.; Ding, Z.; Zhao, Y.; Wang, C.C.; Purnawali, H.; Tang, C. Stimulus-Responsive Shape Memory Materials: A Review. Mater. Des. 2012, 33, 577-640. [CrossRef]

109. Sokolowski, W.; Metcalfe, A.; Hayashi, S.; Yahia, L.; Raymond, J. Medical Applications of Shape Memory Polymers. Biomed. Mater. 2007, 2, S23-S27. [CrossRef] [PubMed]

110. Ware, T.; Simon, D.; Hearon, K.; Liu, C.; Shah, S.; Reeder, J.; Khodaparast, N.; Kilgard, M.P.; Maitland, D.J.; Rennaker, R.L.; et al. Three-Dimensional Flexible Electronics Enabled by Shape Memory Polymer Substrates for Responsive Neural Interfaces. Macromol. Mater. Eng. 2012, 297, 1193-1202. [CrossRef]

111. Lu, H.; Yu, K.; Liu, Y.; Leng, J. Sensing and Actuating Capabilities of a Shape Memory Polymer Composite Integrated with Hybrid Filler. Smart Mater. Struct. 2010, 19, 065014. [CrossRef]

112. Santo, L.; Quadrini, F.; Accettura, A.; Villadei, W. Shape Memory Composites for Self-Deployable Structures in Aerospace Applications. Procedia Eng. 2014, 88, 42-47. [CrossRef] 
113. Mao, Y.; Yu, K.; Isakov, M.S.; Wu, J.; Dunn, M.L.; Jerry Qi, H. Sequential Self-Folding Structures by 3D Printed Digital Shape Memory Polymers. Sci. Rep. 2015, 5, 13616. [CrossRef]

114. Pandini, S.; Passera, S.; Messori, M.; Paderni, K.; Toselli, M.; Gianoncelli, A.; Bontempi, E.; Riccò, T. Two-Way Reversible Shape Memory Behaviour of Crosslinked Poly( $\epsilon$-Caprolactone). Polymer 2012, 53, 1915-1924. [CrossRef]

115. Ishida, K.; Yoshie, N. Two-Way Conversion between Hard and Soft Properties of Semicrystalline Cross-Linked Polymer. Macromolecules 2008, 41, 4753-4757. [CrossRef]

116. Chung, T.; Romo-Uribe, A.; Mather, P.T. Two-Way Reversible Shape Memory in a Semicrystalline Network. Macromolecules 2008, 41, 184-192. [CrossRef]

117. Qin, H.; Mather, P.T. Combined One-Way and Two-Way Shape Memory in a Glass-Forming Nematic Network. Macromolecules 2009, 42, 273-280. [CrossRef]

118. Pilate, F.; Toncheva, A.; Dubois, P.; Raquez, J.M. Shape-Memory Polymers for Multiple Applications in the Materials World. Eur. Polym. J. 2016, 80, 268-294. [CrossRef]

119. Scalet, G. Two-Way and Multiple-Way Shape Memory Polymers for Soft Robotics: An Overview. Actuators 2020, 9, 10. [CrossRef]

120. Xia, Y.; He, Y.; Zhang, F.; Liu, Y.; Leng, J. A Review of Shape Memory Polymers and Composites: Mechanisms, Materials, and Applications. Adv. Mater. 2020, 2000713. [CrossRef]

121. Yang, Z.; Zhang, L. Magnetic Actuation Systems for Miniature Robots: A Review. Adv. Intell. Syst. 2020, 2000082. [CrossRef]

122. Sitti, M.; Wiersma, D.S. Pros and Cons: Magnetic versus Optical Microrobots. Adv. Mater. 2020, 32, 1906766. [CrossRef]

123. Xu, T.; Zhang, J.; Salehizadeh, M.; Onaizah, O.; Diller, E. Millimeter-Scale Flexible Robots with Programmable Three-Dimensional Magnetization and Motions. Sci. Robot. 2019, 4. [CrossRef]

124. O'Halloran, A.; O'Malley, F.; McHugh, P. A Review on Dielectric Elastomer Actuators, Technology, Applications, and Challenges. J. Appl. Phys. 2008, 104, 071101. [CrossRef]

125. Ohm, C.; Brehmer, M.; Zentel, R. Liquid Crystalline Elastomers as Actuators and Sensors. Adv. Mater. 2010, 22, 3366-3387. [CrossRef] [PubMed]

126. Jiang, Z.C.; Xiao, Y.Y.; Zhao, Y. Shining Light on Liquid Crystal Polymer Networks: Preparing, Reconfiguring, and Driving Soft Actuators. Adv. Opt. Mater. 2019, 7, 1900262. [CrossRef]

127. Ge, F.; Zhao, Y. Microstructured Actuation of Liquid Crystal Polymer Networks. Adv. Funct. Mater. 2020, 30, 1901890. [CrossRef]

128. Suo, Z. Theory of Dielectric Elastomers. Acta Mech. Solida Sin. 2010, 23, 549-578. [CrossRef]

129. Gupta, U.; Qin, L.; Wang, Y.; Godaba, H.; Zhu, J. Soft Robots Based on Dielectric Elastomer Actuators: A Review. Smart Mater. Struct. 2019, 28, 103002. [CrossRef]

130. Duduta, M.; Hajiesmaili, E.; Zhao, H.; Wood, R.J.; Clarke, D.R. Realizing the Potential of Dielectric Elastomer Artificial Muscles. Proc. Natl. Acad. Sci. USA 2019, 116, 2476-2481. [CrossRef]

131. Gorissen, B.; Reynaerts, D.; Konishi, S.; Yoshida, K.; Kim, J.W.; De Volder, M. Elastic Inflatable Actuators for Soft Robotic Applications. Adv. Mater. 2017, 29, 1604977. [CrossRef]

132. Polygerinos, P.; Correll, N.; Morin, S.A.; Mosadegh, B.; Onal, C.D.; Petersen, K.; Cianchetti, M.; Tolley, M.T.; Shepherd, R.F. Soft Robotics: Review of Fluid-Driven Intrinsically Soft Devices; Manufacturing, Sensing, Control, and Applications in Human-Robot Interaction: Review of Fluid-Driven Intrinsically Soft Robots. Adv. Eng. Mater. 2017, 19, 1700016. [CrossRef]

133. Lambert, P.; Szewczyk, J.; Renaud, P. Actionneurs Non Conventionnels Pour la Robotique. Techniques de l'ingénieur Conception, modélisation et commande en robotique. 2016, s7769. Available online: https: / / www.techniques-ingenieur.fr/base-documentaire/automatique-robotique-th16/conceptionmodelisation-et-commande-en-robotique-42398210/actionneurs-non-conventionnels-pour-la-robotiques7769/ (accessed on 2 August 2020).

134. Walker, J.; Zidek, T.; Harbel, C.; Yoon, S.; Strickland, F.S.; Kumar, S.; Shin, M. Soft Robotics: A Review of Recent Developments of Pneumatic Soft Actuators. Actuators 2020, 9, 3. [CrossRef]

135. Mustaza, S.M.; Elsayed, Y.; Lekakou, C.; Saaj, C.; Fras, J. Dynamic Modeling of Fiber-Reinforced Soft Manipulator: A Visco-Hyperelastic Material-Based Continuum Mechanics Approach. Soft Robot. 2019, 6, 305-317. [CrossRef] 
136. Joshi, S.; Paik, J. Pneumatic Supply System Parameter Optimization for Soft Actuators. Soft Robot. 2020. [CrossRef] [PubMed]

137. Wehner, M.; Truby, R.L.; Fitzgerald, D.J.; Mosadegh, B.; Whitesides, G.M.; Lewis, J.A.; Wood, R.J. An Integrated Design and Fabrication Strategy for Entirely Soft, Autonomous Robots. Nature 2016, 536, 451-455. [CrossRef] [PubMed]

138. Miriyev, A.; Caires, G.; Lipson, H. Functional Properties of Silicone/Ethanol Soft-Actuator Composites. Mater. Des. 2018, 145, 232-242. [CrossRef]

139. Li, X.; Duan, H.; Lv, P.; Yi, X. Soft Actuators Based on Liquid-Vapor Phase Change Composites. Soft Robot. 2020. [CrossRef] [PubMed]

140. Miriyev, A.; Trujillo, C.; Caires, G.; Lipson, H. Rejuvenation of Soft Material-Actuator. MRS Commun. 2018, 8, 556-561. [CrossRef]

141. Tian, Y.; Li, Y.T.; Tian, H.; Yang, Y.; Ren, T.L. Recent Progress of Soft Electrothermal Actuators. Soft Robot. 2020. [CrossRef] [PubMed]

142. Koetting, M.C.; Peters, J.T.; Steichen, S.D.; Peppas, N.A. Stimulus-Responsive Hydrogels: Theory, Modern Advances, and Applications. Mater. Sci. Eng. R Rep. 2015, 93, 1-49. [CrossRef]

143. Mohd Jani, J.; Leary, M.; Subic, A.; Gibson, M.A. A Review of Shape Memory Alloy Research, Applications and Opportunities. Mater. Des. 2014, 56, 1078-1113. [CrossRef]

144. Haga, Y.; Mizushima, M.; Matsunaga, T.; Totsu, K.; Esashi, M. Active Bending Ileus Tube Using Shape Memory Alloy for Treatment of Intestinal Obstruction. In Proceedings of the 2005 3rd IEEE/EMBS Special Topic Conference on Microtechnology in Medicine and Biology, Oahu, HI, USA, 12-15 May 2005; pp. 249-252. [CrossRef]

145. Kianzad, S.; Amini, A.; Karkouti, S.O. Force Control of Laparoscopy Grasper Using Antagonistic Shape Memory Alloy. In Proceedings of the 2011 1st Middle East Conference on Biomedical Engineering, Sharjah, UAE, 21-24 February 2011; pp. 335-338. [CrossRef]

146. Gao, X.; Yang, J.; Wu, J.; Xin, X.; Li, Z.; Yuan, X.; Shen, X.; Dong, S. Piezoelectric Actuators and Motors: Materials, Designs, and Applications. Adv. Mater. Technol. 2020, 5, 1900716. [CrossRef]

147. Deng, T.; Wang, H.; Chen, W.; Wang, X.; Pfeifer, R. Development of a New Cable-Driven Soft Robot for Cardiac Ablation. In Proceedings of the 2013 IEEE International Conference on Robotics and Biomimetics (ROBIO), Shenzhen, China, 12-14 December 2013; pp. 728-733. [CrossRef]

148. Gerboni, G.; Henselmans, P.W.J.; Arkenbout, E.A.; van Furth, W.R.; Breedveld, P. HelixFlex: Bioinspired Maneuverable Instrument for Skull Base Surgery. Bioinspir. Biomim. 2015, 10, 066013. [CrossRef]

149. Feng, F.; Hong, W.; Xie, L. Design of 3D-Printed Flexible Joints With Presettable Stiffness for Surgical Robots. IEEE Access 2020, 8, 79573-79585. [CrossRef]

150. Dupont, P.E.; Lock, J.; Itkowitz, B.; Butler, E. Design and Control of Concentric-Tube Robots. IEEE Trans. Robot. 2010, 26, 209-225. [CrossRef] [PubMed]

151. Qiu, T.; Palagi, S.; Mark, A.G.; Melde, K.; Adams, F.; Fischer, P. Wireless Actuation with Functional Acoustic Surfaces. Appl. Phys. Lett. 2016, 109, 191602. [CrossRef]

152. Taniguchi, T.; Sugiyama, H.; Uekusa, H.; Shiro, M.; Asahi, T.; Koshima, H. Walking and Rolling of Crystals Induced Thermally by Phase Transition. Nat. Commun. 2018, 9, 538. [CrossRef] [PubMed]

153. Appiah, C.; Arndt, C.; Siemsen, K.; Heitmann, A.; Staubitz, A.; Selhuber-Unkel, C. Living Materials Herald a New Era in Soft Robotics. Adv. Mater. 2019, 31, 1807747. [CrossRef]

154. Webster-Wood, V.A.; Akkus, O.; Gurkan, U.A.; Chiel, H.J.; Quinn, R.D. Organismal Engineering: Toward a Robotic Taxonomic Key for Devices Using Organic Materials. Sci. Robot. 2017, 2, eaap9281. [CrossRef]

155. Blanc, L.; Delchambre, A.; Lambert, P. Flexible Medical Devices: Review of Controllable Stiffness Solutions. Actuators 2017, 6, 23. [CrossRef]

156. Kuder, I.K.; Arrieta, A.F.; Raither, W.E.; Ermanni, P. Variable Stiffness Material and Structural Concepts for Morphing Applications. Prog. Aerosp. Sci. 2013, 63, 33-55. [CrossRef]

157. Loeve, A.; Breedveld, P.; Dankelman, J. Scopes Too Flexible...and Too Stiff. IEEE Pulse 2010, 1, $26-41$. [CrossRef]

158. Manti, M.; Cacucciolo, V.; Cianchetti, M. Stiffening in Soft Robotics: A Review of the State of the Art. IEEE Robot. Autom. Mag. 2016, 23, 93-106. [CrossRef] 
159. Yoshida, S.; Morimoto, Y.; Zheng, L.; Onoe, H.; Takeuchi, S. Multipoint Bending and Shape Retention of a Pneumatic Bending Actuator by a Variable Stiffness Endoskeleton. Soft Robot. 2018, 5, 718-725. [CrossRef] [PubMed]

160. Cartolano, M.; Xia, B.; Miriyev, A.; Lipson, H. Conductive Fabric Heaters for Heat-Activated Soft Actuators. Actuators 2019, 8, 9. [CrossRef]

161. Bilodeau, R.A.; Miriyev, A.; Lipson, H.; Kramer-Bottiglio, R. All-Soft Material System for Strong Soft Actuators. In Proceedings of the 2018 IEEE International Conference on Soft Robotics (RoboSoft), Livorno, Italy, 24-28 April 2018; pp. 288-294. [CrossRef]

162. Mishra, S.R.; Tracy, J.B. Sequential Actuation of Shape-Memory Polymers through Wavelength-Selective Photothermal Heating of Gold Nanospheres and Nanorods. ACS Appl. Nano Mater. 2018, 1, 3063-3067. [CrossRef]

163. Nguyen, B.H.; Nguyen, V.H.; Tran, H.N. Rich Variety of Substrates for Surface Enhanced Raman Spectroscopy. Adv. Nat. Sci. Nanosci. Nanotechnol. 2016, 7, 033001. [CrossRef]

164. Li, J.F.; Li, Z.Y. Manipulation of Plasmonic Wavefront and Light-Matter Interaction in Metallic Nanostructures: A Brief Review. Chin. Phys. B 2014, 23, 047305. [CrossRef]

165. Luo, H.; Li, Z.; Yi, G.; Wang, Y.; Zu, X.; Wang, H.; Huang, H.; Liang, Z. Temperature Sensing of Conductive Shape Memory Polymer Composites. Mater. Lett. 2015, 140, 71-74. [CrossRef]

166. Luo, H.; Li, Z.; Yi, G.; Zu, X.; Wang, H.; Wang, Y.; Huang, H.; Hu, J.; Liang, Z.; Zhong, B. Electro-Responsive Silver Nanowire-Shape Memory Polymer Composites. Mater. Lett. 2014, 134, 172-175. [CrossRef]

167. Weems, A.C.; Raymond, J.E.; Easley, A.D.; Wierzbicki, M.A.; Gustafson, T.; Monroe, M.B.B.; Maitland, D.J. Shape Memory Polymers with Visible and Near-Infrared Imaging Modalities: Synthesis, Characterization and in Vitro Analysis. RSC Adv. 2017, 7, 19742-19753. [CrossRef]

168. Yang, L.; Lu, X.; Wang, Z.; Xia, H. Diels-Alder Dynamic Crosslinked Polyurethane/Polydopamine Composites with NIR Triggered Self-Healing Function. Polym. Chem. 2018, 9, 2166-2172. [CrossRef]

169. Zhang, H.; Zhao, Y. Polymers with Dual Light-Triggered Functions of Shape Memory and Healing Using Gold Nanoparticles. ACS Appl. Mater. Interfaces 2013, 5, 13069-13075. [CrossRef]

170. Fang, T.; Cao, L.; Chen, S.; Fang, J.; Zhou, J.; Fang, L.; Lu, C.; Xu, Z. Preparation and Assembly of Five Photoresponsive Polymers to Achieve Complex Light-Induced Shape Deformations. Mater. Des. 2018, 144, 129-139. [CrossRef]

171. Zhang, Z.X.; Dou, J.X.; He, J.H.; Xiao, C.X.; Shen, L.Y.; Yang, J.H.; Wang, Y.; Zhou, Z.W. Electrically/Infrared Actuated Shape Memory Composites Based on a Bio-Based Polyester Blend and Graphene Nanoplatelets and Their Excellent Self-Driven Ability. J. Mater. Chem. C 2017, 5, 4145-4158. [CrossRef]

172. Zhang, H.; Zhang, J.; Tong, X.; Ma, D.; Zhao, Y. Light Polarization-Controlled Shape-Memory Polymer/Gold Nanorod Composite. Macromol. Rapid Commun. 2013, 34, 1575-1579. [CrossRef] [PubMed]

173. Lu, H.; Zhu, S.; Yang, Y.; Huang, W.M.; Leng, J.; Du, S. Surface Grafting of Carbon Fibers with Artificial Silver-Nanoparticle-Decorated Graphene Oxide for High-Speed Electrical Actuation of Shape-Memory Polymers. J. Appl. Polym. Sci. 2014. [CrossRef]

174. Lu, H.; Liang, F.; Gou, J.; Leng, J.; Du, S. Synergistic Effect of Ag Nanoparticle-Decorated Graphene Oxide and Carbon Fiber on Electrical Actuation of Polymeric Shape Memory Nanocomposites. Smart Mater. Struct. 2014, 23, 085034. [CrossRef]

175. Li, J.; Sun, M.; Wu, Z. Design and Fabrication of a Low-Cost Silicone and Water-Based Soft Actuator with a High Load-to-Weight Ratio. Soft Robot. 2020. [CrossRef]

176. Zhao, T.; Dou, W.; Hu, Z.; Hou, W.; Sun, Y.; Lv, J.A. Reconfigurable Soft Actuators with Multiple-Stimuli Responses. Macromol. Rapid Commun. 2020, 41, 2000313. [CrossRef]

177. Armon, S.; Efrati, E.; Kupferman, R.; Sharon, E. Geometry and Mechanics in the Opening of Chiral Seed Pods. Science 2011, 333, 1726-1730. [CrossRef]

178. Connolly, F.; Walsh, C.J.; Bertoldi, K. Automatic Design of Fiber-Reinforced Soft Actuators for Trajectory Matching. Proc. Natl. Acad. Sci. USA 2017, 114, 51-56. [CrossRef]

179. Connolly, F.; Polygerinos, P.; Walsh, C.J.; Bertoldi, K. Mechanical Programming of Soft Actuators by Varying Fiber Angle. Soft Robot. 2015, 2, 26-32. [CrossRef]

180. Paez, L.; Agarwal, G.; Paik, J. Design and Analysis of a Soft Pneumatic Actuator with Origami Shell Reinforcement. Soft Robot. 2016, 3, 109-119. [CrossRef] 
181. Wang, Z.; Polygerinos, P.; Overvelde, J.T.B.; Galloway, K.C.; Bertoldi, K.; Walsh, C.J. Interaction Forces of Soft Fiber Reinforced Bending Actuators. IEEE/ASME Trans. Mechatronics 2017, 22, 717-727. [CrossRef]

182. Lazeroms, M.; La Haye, A.; Sjoerdsma, W.; Schreurs, W.; Jongkind, W.; Honderd, G.; Grimbergen, C. A Hydraulic Forceps with Force-Feedback for Use in Minimally Invasive Surgery. Mechatronics 1996, 6, 437-446. [CrossRef]

183. Huber, J.E.; Fleck, N.A.; Ashby, M.F. The Selection of Mechanical Actuators Based on Performance Indices. Proc. R. Soc. Lond. A 1997, 453, 2185-2205. [CrossRef]

184. Miriyev, A. A Focus on Soft Actuation. Actuators 2019, 8, 74. [CrossRef]

185. Blanc, L. Controllable Stiffness Mechanisms for Endoscopic Catheters. Ph.D. Thesis, Université libre de Bruxelles, Bruxelles, Belgium, 2019.

186. McKee, C.T.; Last, J.A.; Russell, P.; Murphy, C.J. Indentation Versus Tensile Measurements of Young's Modulus for Soft Biological Tissues. Tissue Eng. Part B Rev. 2011, 17, 155-164. [CrossRef]

187. Liu, J.; Sidiropoulos, A.; Konertz, W. Minimally Invasive Aortic Valve Replacement (AVR) Compared to Standard AVR. Eur. J. Cardiothorac. Surg. 1999, 16, S80-S83._2.S80. [CrossRef]

188. Shah, D.; Yang, B.; Kriegman, S.; Levin, M.; Bongard, J.; Kramer-Bottiglio, R. Shape Changing Robots: Bioinspiration, Simulation, and Physical Realization. Adv. Mater. 2020, 2002882. [CrossRef]

189. Odent, J.; Toncheva, A.; Dubois, P.; Raquez, J.M. From 3D to 4D-Printing: On the Road to Smart 3D-Printed Polymer Devices. In How Smart Are the Polymers?; Polymer Science and Technology, Nova Science Publishers: New York, NY, USA, 2018.

190. Allen, E.; Swensen, J. Directional Stiffness Control Through Geometric Patterning and Localized Heating of Field's Metal Lattice Embedded in Silicone. Actuators 2018, 7, 80. [CrossRef]

191. Bertoldi, K.; Vitelli, V.; Christensen, J.; van Hecke, M. Flexible Mechanical Metamaterials. Nat. Rev. Mater. 2017, 2, 17066. [CrossRef]

192. Ren, X.; Das, R.; Tran, P.; Ngo, T.D.; Xie, Y.M. Auxetic Metamaterials and Structures: A Review. Smart Mater. Struct. 2018, 27, 023001. [CrossRef]

193. Fischer, S.C.L.; Hillen, L.; Eberl, C. Mechanical Metamaterials on the Way from Laboratory Scale to Industrial Applications: Challenges for Characterization and Scalability. Materials 2020, 13, 3605. [CrossRef] [PubMed]

194. Nam, S.; Pei, E. A Taxonomy of Shape-Changing Behavior for 4D Printed Parts Using Shape-Memory Polymers. Prog. Addit. Manuf. 2019, 4, 167-184. [CrossRef]

195. Kim, S.; Laschi, C.; Trimmer, B. Soft Robotics: A Bioinspired Evolution in Robotics. Trends Biotechnol. 2013, 31, 287-294. [CrossRef] [PubMed]

196. Shie, M.Y.; Shen, Y.F.; Astuti, S.D.; Lee, A.K.X.; Lin, S.H.; Dwijaksara, N.L.B.; Chen, Y.W. Review of Polymeric Materials in 4D Printing Biomedical Applications. Polymers 2019, 11, 1864. [CrossRef]

197. Ashuri, T.; Armani, A.; Jalilzadeh Hamidi, R.; Reasnor, T.; Ahmadi, S.; Iqbal, K. Biomedical Soft Robots: Current Status and Perspective. Biomed. Eng. Lett. 2020, 10, 369-385. [CrossRef]

198. Runciman, M.; Darzi, A.; Mylonas, G.P. Soft Robotics in Minimally Invasive Surgery. Soft Robot. 2019, 6, 423-443. [CrossRef]

199. De Greef, A.; Lambert, P.; Delchambre, A. Towards Flexible Medical Instruments: Review of Flexible Fluidic Actuators. Precis. Eng. 2009, 33, 311-321. [CrossRef]

200. Field, R.D.; Anandakumaran, P.N.; Sia, S.K. Soft Medical Microrobots: Design Components and System Integration. Appl. Phys. Rev. 2019, 6, 041305. [CrossRef]

201. Hughes, J.; Culha, U.; Giardina, F.; Guenther, F.; Rosendo, A.; Iida, F. Soft Manipulators and Grippers: A Review. Front. Robot. AI 2016, 3. [CrossRef]

202. Fenci, G.E.; Currie, N.G. Deployable Structures Classification: A Review. Int. J. Space Struct. 2017, 32, 112-130. [CrossRef]

203. Chowdhary, G.; Gazzola, M.; Krishnan, G.; Soman, C.; Lovell, S. Soft Robotics as an Enabling Technology for Agroforestry Practice and Research. Sustainability 2019, 11, 6751. [CrossRef]

204. Arezzo, A.; Mintz, Y.; Allaix, M.E.; Arolfo, S.; Bonino, M.; Gerboni, G.; Brancadoro, M.; Cianchetti, M.; Menciassi, A.; Wurdemann, H.; et al. Total Mesorectal Excision Using a Soft and Flexible Robotic Arm: A Feasibility Study in Cadaver Models. Surg. Endosc. 2017, 31, 264-273. [CrossRef] [PubMed]

205. Yeung, C.K.; Cheung, J.L.; Sreedhar, B. Emerging Next-Generation Robotic Colonoscopy Systems towards Painless Colonoscopy. J. Dig. Dis. 2019, 20, 196-205. [CrossRef] [PubMed] 
206. Rus, D.; Tolley, M.T. Design, Fabrication and Control of Origami Robots. Nat. Rev. Mater. 2018, 3, 101-112. [CrossRef]

207. van Manen, T.; Janbaz, S.; Zadpoor, A.A. Programming the Shape-Shifting of Flat Soft Matter. Mater. Today 2018, 21, 144-163. [CrossRef]

208. Joshi, S.; Rawat, K.; Karunakaran, C.; Rajamohan, V.; Mathew, A.T.; Koziol, K.; Kumar Thakur, V.; A.S.S, B. 4D Printing of Materials for the Future: Opportunities and Challenges. Appl. Mater. Today 2020, 18, 100490. [CrossRef]

209. Mitchell, A.; Lafont, U.; Hołyńska, M.; Semprimoschnig, C. Additive Manufacturing-A Review of 4D Printing and Future Applications. Addit. Manuf. 2018, 24, 606-626. [CrossRef]

210. Huang, X.; Ford, M.; Patterson, Z.J.; Zarepoor, M.; Pan, C.; Majidi, C. Shape Memory Materials for Electrically-Powered Soft Machines. J. Mater. Chem. B 2020, 8, 4539-4551. [CrossRef]

211. Wallin, T.J.; Pikul, J.; Shepherd, R.F. 3D Printing of Soft Robotic Systems. Nat. Rev. Mater. 2018, 3, 84-100. [CrossRef]

212. Truby, R.L.; Lewis, J.A. Printing Soft Matter in Three Dimensions. Nature 2016, 540, 371-378. [CrossRef] [PubMed]

213. Gul, J.Z.; Sajid, M.; Rehman, M.M.; Siddiqui, G.U.; Shah, I.; Kim, K.H.; Lee, J.W.; Choi, K.H. 3D Printing for Soft Robotics-A Review. Sci. Technol. Adv. Mater. 2018, 19, 243-262. [CrossRef] [PubMed]

Publisher's Note: MDPI stays neutral with regard to jurisdictional claims in published maps and institutional affiliations. 